DOI: $10.3901 / J M E .2021 .08 .023$

\title{
先进光学制造技术最新进展
}

王振忠 $^{1}$ 施晨淳 $^{1}$ 张鹏飞 $^{2}$ 杨 $^{\text {哲 }}{ }^{2}$ 陈 $_{\text {熠 }}{ }^{1}$ 郭 $^{\text {江 }^{2}}$

(1. 厦门大学航空航天学院 厦门 361005;

2. 大连理工大学机械工程学院 大连 116024)

摘要: 在激光核聚变、大型天文望远镜等国家大光学工程及各种光机电产品的驱动下, 高面形精度、高表面质量、多结构型 式光学组件的需求量日益增加, 因此, 先进光学制造技术显得尤为重要。主要综述了近十年来光学超精密加工技术的发展情 况, 主要包括超精密车削、磨削和抛光技术。根据光学组件的材料特性、结构特征和加工要求等, 阐述了超精密加工技术的 具体研究进展, 包括传统技术的迭代更新与新型技术的研制开发, 并针对典型应用进行举例。最后, 展望了超精密光学加工 技术的发展趋势。希望能为光学制造领域后续深入研究提供参考。

关键词: 光学; 超精密加工; 加工机理；机床设备；光学组件

中图分类号: TH161

\section{Recent Progress of Advanced Optical Manufacturing Technology}

\author{
WANG Zhenzhong $^{1}$ SHI Chenchun ${ }^{1}$ ZHANG Pengfei ${ }^{2} \quad$ YANG Zhe $^{2}$ \\ CHEN Yi ${ }^{1}$ GUO Jiang ${ }^{2}$
}

(1. School of Aeronautics and Astronautics, Xiamen University, Xiamen 361005;

2. School of Mechanical Engineering, Dalian University of Technology, Dalian 116024)

\begin{abstract}
Driven by various optical and mechanical products, as well as the national major optical projects such as laser nuclear fusion and large astronomical telescopes, the demand for those optical components with high surface accuracy, high surface quality, and multiple types and sizes are increasing. Accordingly, the advanced optical manufacturing technology does become particularly critical. In this paper, the development of advanced optical manufacturing technology in recent ten years is reviewed, including ultra precision turning technology, ultra precision grinding technology and ultra precision polishing technology. Based on the material characteristics, machining requirements and structures of the optical elements, the research progress of ultra precision machining technology is specifically described, there are not only the development and renewal of traditional technology, but also the appearance of new technology, some typical applications also are given. Lastly, the development trend of ultra precision optical machining technology is prospected. It aims to provide a valuable reference for the peer researchers.
\end{abstract}

Key words: optics; ultra precision machining; machining mechanism; machine tool; optical element

\section{0 前言}

近年来，在激光聚变国家重大科学工程、高分 辨对地观测重大科技专项及各种光机电产品需求日 益增长的推动下, 光学组件的超精密加工正向着高 精度、高效率、低成本方向发展。以晶体 $\mathrm{KDP} 、 \mathrm{CaF}_{2}$ 、

20201212 收到初稿, 20210322 收到修改稿
FCD100 光学玻璃等为代表的光学材料已广泛应用 于光学成像、大功率激光系统、激光核聚变、太阳 能电池、空间观测、传感器、探测器等领域, 需求 量巨大 ${ }^{[1-3]}$ 。光学组件的面形精度和表面质量始终是 备受关注的两大要素, 前者决定其功能, 后者决定 其性能。虽然加工方法多样，但能够同时保证高面 形精度和高表面质量的光学加工技术却很少。除此 之外, 加工后的光学组件表面还要求有尽可能少的 
表面/亚表面损伤, 这也是目前超精密加工领域共同 面临的难题。所以, 光学加工是一项很难的加工技 术, 这也正是一个国家超精密加工技术水平的重要 体现 $^{[4-5]}$ 。

随着大型空间光学遥感技术以及深空天体观测 需求的不断发展, 光学系统中的非球面反射镜性能 要求越来越高, 主要表现在: 口径越来越大, 已 经提高至 $4 \mathrm{~m}$ 量级; 轻量化率越来越高, 从以往 的 $60 \%$ 提高至 $85 \%$; 面形精度要求越来越高, 从 $0.020 \lambda \mathrm{RMS}$ 提高至 $0.013 \lambda \mathrm{RMS}$ 。因此, 加工获得 高精度、轻量化、高比刚度的大口径光学组件成为 了难题。例如, 碳化硅作为空间光学组件反射镜镜 坏的材料之一而广泛应用于空间相机反射镜, 但因 其硬度大、加工难度较高, 严重影响反射镜的加工 周期, 并且在大口径碳化硅非球面反射镜的磨削过 程中, 砂轮磨损相对严重, 非球面反射镜加工精度 和表面质量受到直接影响 ${ }^{[6-7]}$ 。

而对于小口径非球面和自由曲面来说, 其加工 精度要求亚纳米、深亚纳米甚至皮米量级, 同时要 求加工表面超光滑、无亚表面损伤和超低缺陷。对 于小口径非球面模具的加工, 目前主要采用超精密 金刚石车削和超精密磨削, 但不可避免地会在表面 造成缺陷及损伤, 这些表面缺陷及损伤严重制约了 光学组件的使用性能。对于非球面抛光, 产业界目 前常用手工抛光和黏弹性抛光头结合游离磨料抛 光。不同形状、不同尺寸的工件需要制作专门的抛 光工具, 因此不能提供一个适应性强、效率高的光 整加工方法。特别是对于微小凹型非球面等复杂形 状的工件, 由于其加工空间狭小, 加工工具难以对 其进行有效抛光。所以, 无论是利用黏弹性抛光还 是手工抛光, 尽管表面粗糙度可以得到改善, 但是 在加工效率、加工精度、自动化以及适用的加工面 形等方面都难以满足现代光电技术的发展要 求 $^{[8-12]}$ 。

光学微细结构如棱镜结构、微透镜阵列、菲涅 尔透镜结构在很多光学系统中发挥着成像、聚焦和 照明等重要功能。一般要求光学微细结构组件具有 微米级面形精度和纳米/亚纳米级表面粗䊁度, 面形 精度和表面质量仍然是两个关键要素。由于光学微 细结构本身的复杂性, 所以, 保证面形精度并降低 其表面粗䊁度也一直是人们研究的重点和难 点 $^{[13-15]}$ 。

针对大口径碳化硅反射镜自重大的难题, 孟晓
辉等 ${ }^{[16]}$ 建立了基于重力卸载的高精度旋转加工检 测方法, 采用了 6 次旋转和车削相结合的方法对碳 化硅反射镜进行加工，去除重力变形误差后面形精 度达到了 $0.010 \lambda \mathrm{RMS}$ 。徐放 ${ }^{[17]}$ 针对目前大口径反 射镜自重大的难题尝试使用化学改性方法在铝表面 制备镍磷合金膜层，通过单点金刚石车削和古典抛 光相结合的加工方式，成功制备了符合可见光波段 使用要求的铝合金反射镜。刘冬梅等 ${ }^{[10]}$ 使用全口径 弹性抛光和小工具修抛相结合的方法抛光 $\phi 6 \mathrm{~mm}$ 石英玻璃透镜, 实现了较理想的透镜面形精度 $(0.39 \mu \mathrm{m} \mathrm{PV})$ 和较低的表面粗糙度 $(R a 0.0358 \mu \mathrm{m})$ 。 针对微细结构抛光过程中面形和表面质量不易兼顾 的难题, BRINKSMEIER 等 ${ }^{[18]}$ 提出使用圆锥销式和 雉轮式抛光工具对 $\mathrm{V}$ 沟槽和菲涅尔透镜结构进行 抛光。该方法通过对抛光区域供给游离磨粒, 在 微细结构表面获得了较低的粗粘度并较好地维持 了微细结构的面形精度。CHEUNG 等 ${ }^{[19]}$ 采用气囊 工具抛光微细结构, 利用气囊工具对工件产生的 自适应效果, 实现了大范围微细结构表面的精密 抛光，该方法可用于连续性微细结构如微透镜阵列 等的抛光加工。

但是，上述的大部分加工技术都是借助抛光 工具并配合游离磨料实现对目标表面的超精密 加工, 然而因接触造成的表面/亚表面损伤始终无 法避免。一些非接触的抛光方法如离子束、等离 子抛光等虽然能得到具有良好表面完整性的表 面, 但是其加工效率较低。一些光学加工新方法 也正慢慢出现。李敏 ${ }^{[20]}$ 提出了一种剪切增稠的抛 光方法, 使用小工具头对抛物面反射镜进行抛光 可以实现 $R a 4.73 \mathrm{~nm}$ 的表面粗糙度。流体动压抛 光方面, 研究人员不断更新动压实现方式, 实现 了更高的表面质量, 例如 NIKON 和大版大学合 作开发的用于加工球面和非球面的新一代 EEM 装置, 该装置已经成功完成了对 EUVL 系统非球 面光刻物镜的超光滑表面加工, 并且其表面粗粘 度优于 $0.1 \mathrm{~nm}$ RMS, 这代表了流体动压抛光技术 的前沿。

本文综述了近十年光学超精密加工技术的研 究情况, 主要包括超精密切削、超精密磨削、接 触和非接触超精密抛光等技术, 图 1 为先进光学 制造技术总结示意图。针对现有光学超精密加工 技术和新型抛光技术的发展方向进行展望，为未 来超精密光学制造技术的发展提供指导并为同行 提供参考。 


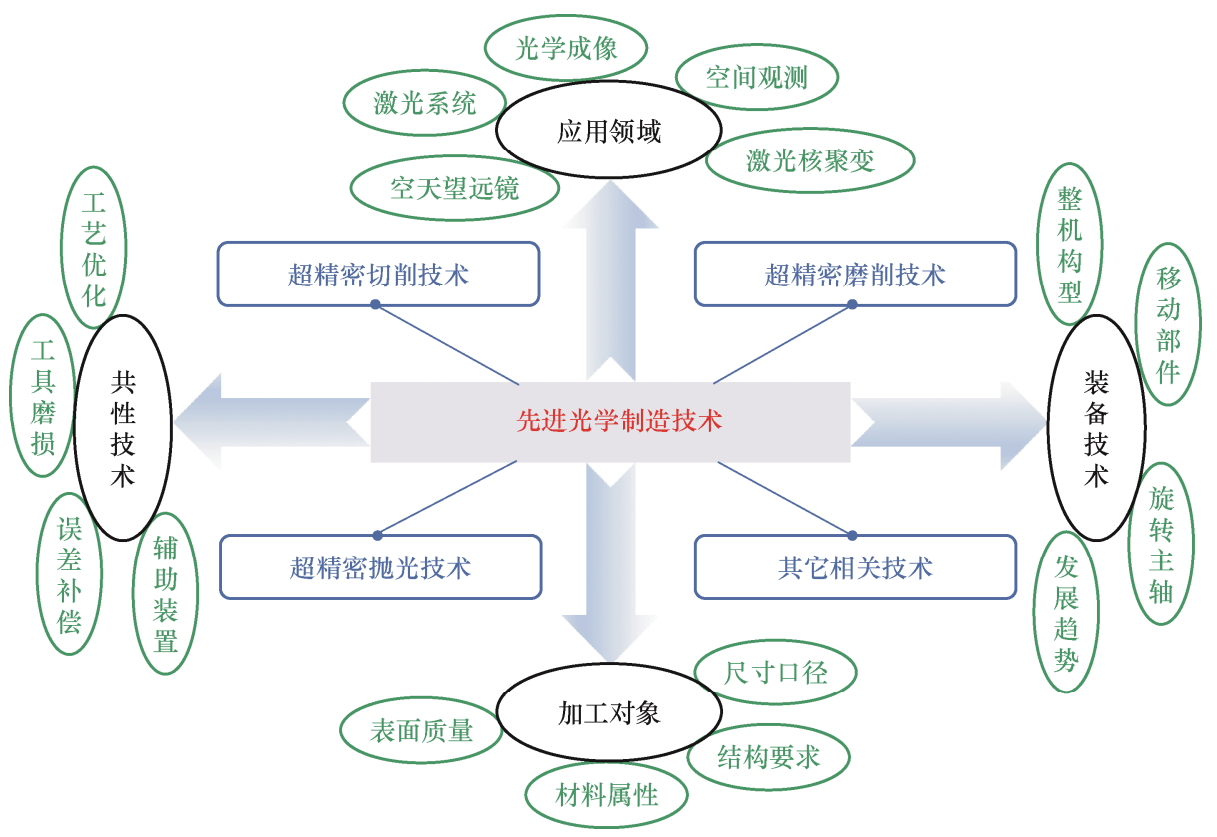

图 1 先进光学制造技术总结示意图

\section{1 超精密切削技术}

超精密切削是先进光学加工的重要技术手段之 一。本节主要综述了单点金刚石车削技术、超精密 铣削技术、飞切技术、伺服系统装置等在光学加工 领域一些新的发展情况。

\section{1 单点金刚石车削技术}

单点金刚石车削是一种超精密加工工艺, 依托 精密机床, 该技术采用金刚石刀具加工光学元 件 $^{[21]}$ 。传统的光学加工方法主要具有加工精度低, 耗时周期长, 技术工人经验依赖强等特点 ${ }^{[22-23]}$ 。与 传统的光学加工技术相比, 单点金刚石车削技术主 要的优点有高效率、高精度、高重复精度等, 因而 该技术广泛应用于光学组件的超精密加工 ${ }^{[24]}$ 。

众多学者基于试验手段对单点金刚石车削技术 进行直接研究。郭海涛 ${ }^{[25]}$ 通过采用正交试验方法进 行 KDP 晶体超精密切削试验设计, 探究随机性背后 隐藏的数学统计规律, 建立影响因素对单点金刚石 切削 KDP 晶体加工表面粗粘度值和质量优劣分布 的预测模型。ZHANG 等 ${ }^{[26]}$ 提出了一种高速单点金 刚石铣削与精密压缩成形相结合的加工方法, 用于 自由曲面非连续微透镜阵列的制备。研究结果表明, 相比慢刀伺服金刚石拉削技术, 高速单点金刚石铣 削在加工不连续特征真实三维光学表面时更具灵活 性。WEI 等 ${ }^{[27]}$ 提出了一种光学自由曲面金刚石车削 的优化刀具轨迹生成方法, 以渐变镜片为加工工件, 并试验验证了方法的有效性。LEI 等 ${ }^{[28]}$ 研究了单点
金刚石切削工艺中真空吸盘孔排列对大口径 KDP 透射波阵面的影响。通过采用新设计的真空吸盘, 有效地改善了真空吸盘的质量, 并相应地抑制了单 点金刚石切削中 KDP 组件出现的边缘效应。 HATEFI 等 ${ }^{[29]}$ 设计开发了用于单点金刚石切削混合 式平台的多轴自动化混合控制器, 能够控制和驱动 超声波振动系统、激光束系统、冷等离子体系统和 在机检测系统，并获得了理想的试验结果。

理论模型与仿真分析也是单点金刚石车削技术 重要的研究手段之一。ZHANG 等 ${ }^{[30]}$ 采用三维分子 动力学模拟方法, 研究了金刚石刀具在纳米尺度下 对单晶硅试件的重复单点切削行为, 进而分析了加 工诱导硅表面纳米切削的相变过程。XU 等 ${ }^{[24]}$ 试验 研究了单点金刚石切削技术的被加工工件表面粗䊁 度的控制策略，系统分析了工件材料特性、刀具参 数、切削参数等, 并成功实现了 $\mathrm{KDP} 、 \mathrm{Si} 、 \mathrm{Ge}$ 等材 料加工的表面粗楉度控制方法。 $\mathrm{HE}$ 等 ${ }^{[31]}$ 提出了一 个创新的表面粗䊁度预报模型, 该模型考虑了运动 学、塑性侧流、材料回弹和随机性等因素。模型揭 示了金刚石车削表面粗粘度尺寸效应的相关机理, 并通过试验验证了理论模型的有效性。HE 等 ${ }^{[2]}$ 综 述了金刚石车削过程中表面形貌的影响因素及相应 的理论模型。ZHANG 等 ${ }^{[33]}$ 基于裂纹相对长度, 通 过考虑材料特性、刀具几何结构、切削参数、液体 静压力抑制等因素, 建立了新型理论模型, 研究并 揭示了刀具前角对 KDP 材料的加工影响规律, 其中 包括裂纹扩展与刀具前角之间的定量关系。

近年来，随着加工技术的不断发展，单点金刚 
石车削复合辅助加工技术在光学加工领域的研究已 不再少见。图 2 为单点金刚石车削辅助加工技术举 例 ${ }^{[34]}$ 。此外, MOHAMMADI 等 ${ }^{[35-36]}$ 采用单点金刚 石切削技术与微激光辅助加工技术结合的方式加工 硅材料(111), 并对激光功率、横向进给速度、刀具 前角等加工参数进行了试验研究, 并对加工后的工 件表面粗糙度进行了分析。结果表明, 采用该技术 可以获得光学质量要求的表面粗䊁度。SHAHINIAN 等 ${ }^{[37]}$ 也研究了微激光辅助单点金刚石切削的加工 技术，但加工材料为熔融石英玻璃等脆硬材料。 试验结果表明, 实现的表面粗楉度最低可至亚纳 米尺度, 实现了该技术的有效性。NAVARE 等 ${ }^{[38]}$ 采用激光辅助金刚石切削技术, 试验研究了金刚 石刀具材料本身的晶体取向对 $\mathrm{ZnS}$ 的加工性能影 响, 关注点包括刀具本身的耐磨性、被加工工件 的加工质量等。ZHOU 等 ${ }^{[39]}$ 集成了单点金刚石切 削法与离子束刻蚀法。采用提出的该方法, 将微 透镜阵列制备于在 $\mathrm{SiC}$ 和 $\mathrm{Si}$ 材料。试验证明了该 新方法的有效性, 并解释了加工机理。LEE 等 ${ }^{[40]}$ 对氟化钙单晶的热辅助微切削技术进行了总结, 主要阐述了热影响工件材料特性变化, 热辅助加 工仿真分析, 热辅助加工具体技术如感应加热、 激光加热、等离子加热等, 并对热加工辅助金刚 石切削技术进行了未来展望。

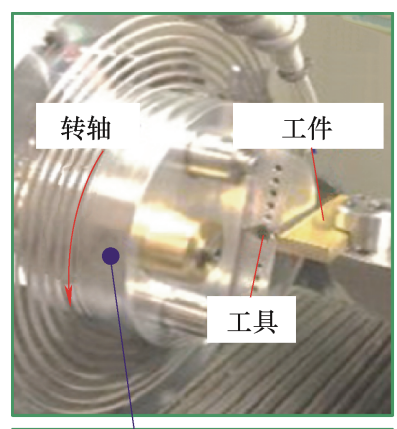

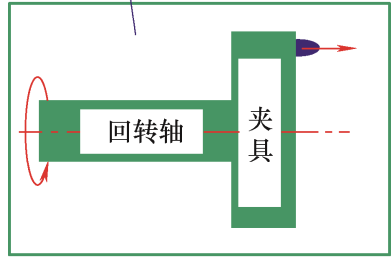

(a) 飞铣
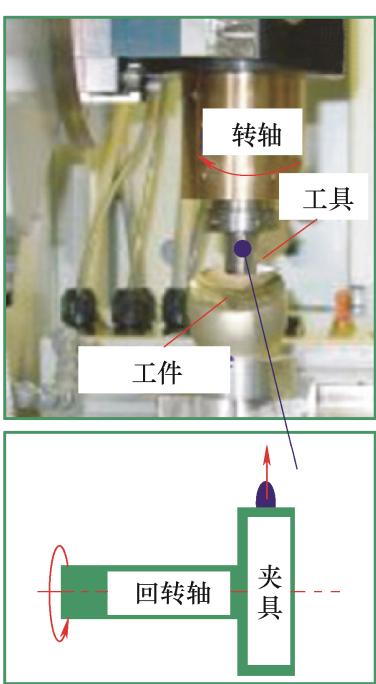

(b) 光栅铣削

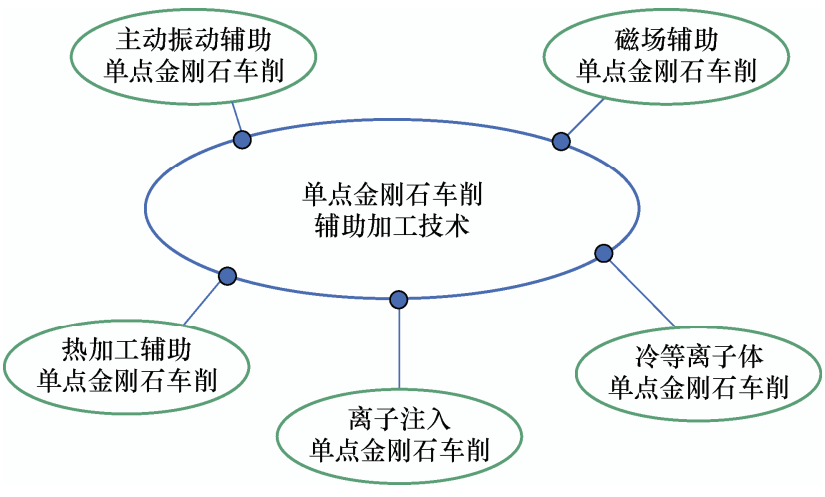

图 2 单点金刚石车削辅助加工技术举例

\section{2 超精密铣削技术}

超精密铣削是有效的光学加工技术之一。超精 密金刚石铣削是从超精密金刚石车削发展而来的, 它涵盖超精密飞切铣削、超精密光栅铣削和超精密 球头铣削等，如图 3 所示 ${ }^{[41]}$ 。与超精密金刚石车削 不同，超精密金刚石铣削是一种间歇切削过程，其 中金刚石工具与主轴作旋转运动。超精密光栅铣削 是基于多轴光栅铣削的超精密自由曲面加工技术, 是一种用以加工非回转对称曲面的有效手段, 加工 具有亚微米形状精度及纳米表面粗䊁度的光学表面 而无需后续处理 ${ }^{[42]}$ 。本节综述了光学超精密铣削加 工技术在加工误差、表面质量、建模仿真、加工系 统设计等方面的新进展。

图 3 超精密金刚石铣削

对于光学组件铣削加工误差的研究, 近些年学 者们多通过建立误差模型并提出误差校正策略来减 少加工误差。ZHANG 等 ${ }^{[43]}$ 研究了超精密光栅铣削 的主轴倾角误差对加工零件的尺寸精度影响规律, 提出了一种基于凹槽切削的新型主轴倾角误差识别
及补偿方法, 并设计了误差补偿程序。试验结果表 明, 该方法能够准确地补偿主轴倾角误差, 有效提 高光学黄铜的加工尺寸精度。 $\mathrm{KONG}$ 等 ${ }^{[44]}$ 通过齐次 变换矩阵建立了加工误差预报模型, 该模型可用于 光学自由曲面超精密光栅铣削成形加工误差的定量 
分析。曹义 ${ }^{[45]}$ 提出一种误差修正方法, 并建立了 刀具误差模型, 如图 4 所示 ${ }^{[45]}$, 该模型主要包括 金刚石刀具相对于旋转轴的径向位移、刀尖半径 误差和刀具前角的刀具不平整度函数等。试验研 究表明, 该方法可以减少自由曲面光学组件的加 工误差。

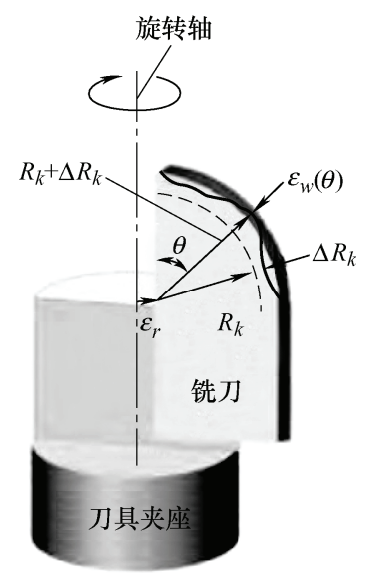

图 4 刀具误差模型

关于光学组件表面质量的改进方法和表面粗粘 度建模等方面均有受到关注。ZHANG 等 ${ }^{[46]}$ 研究了 材料滑动、尺寸效应、刀具磨损等对超精密光栅铣 削表面生成的影响, 研究结果表明, 料滑动会导致 表面微波的形成, 尺寸效应会导致表面粗粘度的增 加, 而刀具磨损会改变刀具的几何形状并进一步影 响表面粗糙度。WANG 等 ${ }^{[47]}$ 研究了超精密铣削过程 中光学材料对表面粗䊁度的影响规律, 在此基础上 提出了一种表征光柱铣削工件表面粗粘度的新方 法。WANG 等 ${ }^{[48]}$ 研究了超精密光栅铣削加工方法对 光学组件曲面质量的影响, 建立了包含切削参数、 刀具轨迹、刀具几何参数、工件尺寸和机床特性等 因素的整体表面粗糙度预测模型, 如图 5 所示 ${ }^{[48]}$, 提出的 3D 表面粗粘度模型可以有效提高自由曲面 的加工质量和效率。

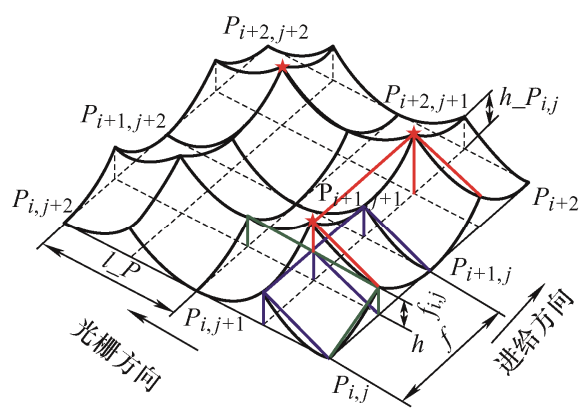

(a)

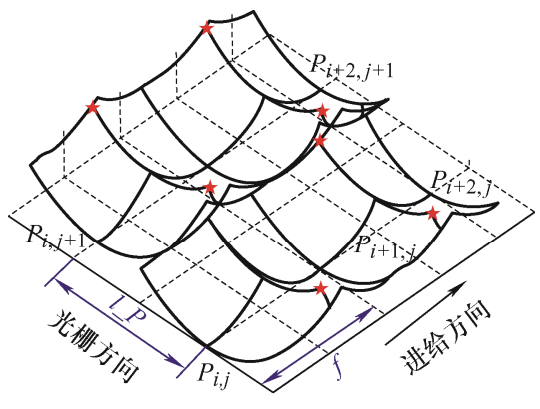

(b)

图 5 三维表面粗楉度模型

此外, 关于光学组件的加工系统设计研究也有 报道。SUN 等 ${ }^{[49]}$ 提出了一种自校正金刚石铣削 (STDM) 系统, 如图 6 所示 ${ }^{[49]}$, 该系统具有高度灵活 的刀具路径规划算法功能, 可以实现更平滑、更均 匀的表面质量。与传统铣削相比, STDM 的自整定 功能可以在确保平滑度的同时大幅提高平均进给速 度, 使加工效率提高近一倍。

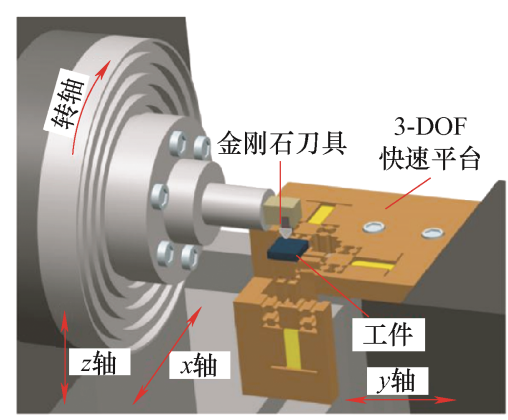

(a)

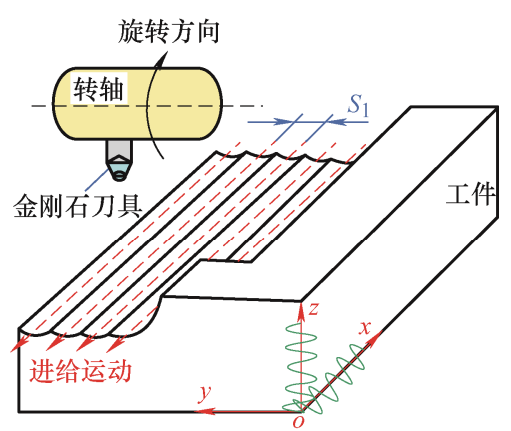

(b)

图 $6 \mathrm{STDM}$ 的硬件配置和切削机理示意图

\section{3 超精密飞切技术}

超精密飞切加工, 是类似用一个刀片作为刀具 的单点铣削加工, 即金刚石刀具安装在高速回转的 主轴径向方向, 同时主轴沿着进给方向间歇性地进 行扫描运动, 工件随着工作台运动, 实现不同方向 的进给, 其原理图如图 7 所示 ${ }^{[50]}$ 。 


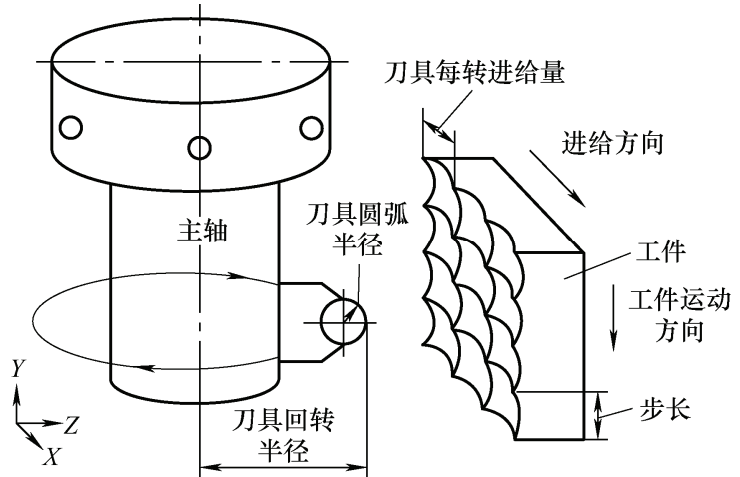

图 7 飞切加工原理示意图

光学组件表面波纹度误差会直接影响其光学性 能, 研究并确定波纹度误差产生的原因, 对提升光 学组件加工精度至关重要。ZHANG 等 ${ }^{[51]}$ 研究了 KDP 晶体的各向异性对切削加工性能的影响规律, 并分析了其他典型工件表面误差产生的原因, 主要 原因包括导轨直线度误差、主轴结构、主轴振动及 其运动误差等。通过对飞切机床的结构和工艺参数 进行优化, 提高了光学组件飞切加工的表面质量。 $\mathrm{FU}$ 等 ${ }^{[52]}$ 通过表面切削仿真研究表明, 刀具的热变 形对 KDP 晶体表面的粗䊁度和波纹度均有影响, 并 会增加工件表面斜率, 从而影响 KDP 晶体表面精 度。CHEN 等 ${ }^{[33]}$ 试验研究了飞切机床主轴振动的频 率范围, 认为光学加工表面波纹度误差产生的原因 与主轴的振动有关。

力学建模与仿真是研究光学加工精度的重要手 段。由于大口径平面光学组件在飞切过程中, 加工 表面会形成中频微波, 极大地影响组件的光学性能。 WANG 等 ${ }^{[54]}$ 建立了超精密飞切主轴系统的有限元 模型, 分析了刀尖的轴向振动。分析表明, 刀尖的 振动频率与主轴系统的结构密切相关, 可通过优化 主轴结构以提高光学加工精度。LU 等 ${ }^{[55]}$ 对刀尖的 振动特性和动力学响应进行了仿真, 并通过试验进 行了验证。通过将刀尖与工件之间的位移转化为三 维曲线, 模拟了被加工表面。结果表明, 飞刀头气 动轴承刚度和切削工艺参数对加工光学表面均会产 生影响。CHEN 等 ${ }^{[56]}$ 基于应变梯度塑性理论模型、 切削力模型以及超精密飞切系统的动态特性, 建立 了 KDP 晶体材料性能影响下的表面形貌预测模型, 并验证了 KDP 晶体材料特性对加工工件的表面粗 粘度有显着影响。

\section{4 伺服系统}

慢速刀具伺服(STS)和快速刀具伺服(FTS)金刚 石切削技术之间的主要区别在于刀具伺服器的行程 和往复频率。FTS 需要一个附加的伺服单元来驱动 刀具, 而在 STS 金刚石切削刀具由机床自身的移动
平台驱动。FTS 加工光学自由曲面的过程如图 8 所

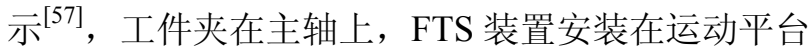
上。在加工前, 对微结构表面形貌数据进行设计和 生成, 并对刀具轨迹进行精确规划和补偿。然后 FTS 驱动金刚石刀具在工件表面沿径向或轴向上产生不 同深度的切削, 最终产生自由曲面。STS 车削原理 图如图 9 所示 ${ }^{[58],} X$ 轴 $Z$ 轴形成 $\mathrm{T}$ 型布局, $C$ 轴固 定在沿 $X$ 轴运动的平台上, 刀具沿 $Z$ 轴运动。在车 削过程中, 工件固定在 $C$ 轴上, 通过同时控制 $X Z C$ 三轴可以实现复杂曲面的加工。

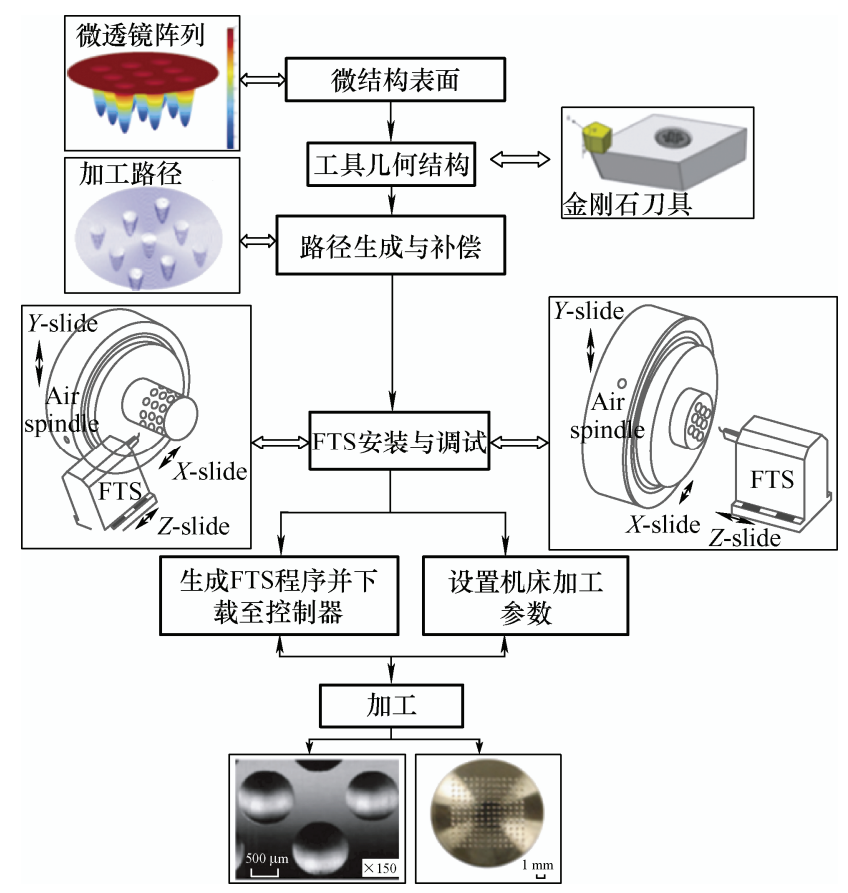

图 8 FTS 加工原理图

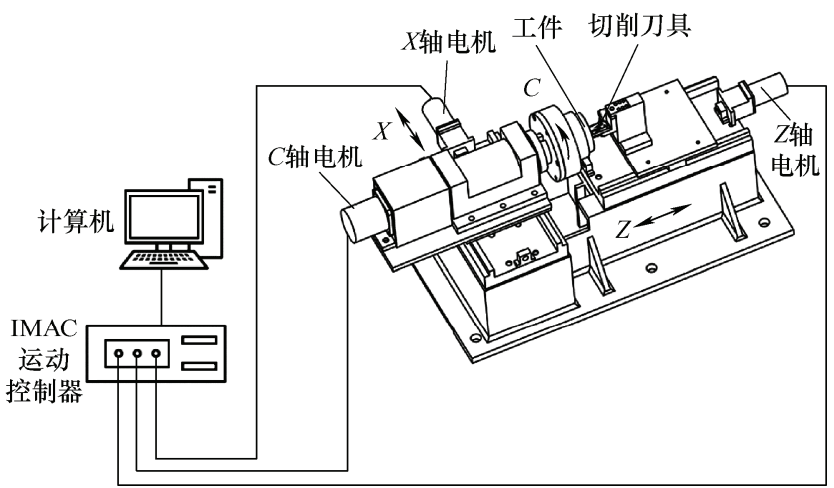

图 $9 \mathrm{STS}$ 加工原理图

由于独特的加工优势, FTS 切削技术已经被广 泛应用于自由曲面车削及微结构加工中。KONG 等 ${ }^{[59]}$ 使用 FTS 实现了口径为 $522.6 \mu \mathrm{m}$ 、深度为 $30 \mu \mathrm{m}$ 的微透镜阵列加工。YU 等 ${ }^{[60]}$ 通过压电驱动 式 FTS 金刚石车削技术在脆性材料上加工了间距为 $600 \mu \mathrm{m}$ 、口径为 $400 \mu \mathrm{m}$ 、高度为 $4 \mu \mathrm{m}$ 的微透镜阵 列, 并获得了表面粗粘度 $R a$ 为 $12 \mathrm{~nm}$ 的无损伤高 
质量表面。LIU 等 ${ }^{[61]}$ 针对 FTS 金刚石车削技术提出 了自由曲面误差补偿切削方法, 获得了形状误差 $P V$ 为 $2.0 \mu \mathrm{m}$ 、表面粗䊁度 $R a$ 为 $36 \mathrm{~nm}$ 的良好表面。 LI 等 ${ }^{[62]}$ 使用 FTS 进行超精密车削高效生成具有光 学表面质量的光学自由曲面, 获得了微米级的形状 误差和 $5.41 \mathrm{~nm}$ 的表面粗糙度。ZHANG 等 ${ }^{[63]}$ 基于 FTS 加工了大纵横比矩形自由曲面, 实现了 $P V$ 为 $380 \mathrm{~nm}$ 亚微米形状精度和 $R a$ 为 $5 \mathrm{~nm}$ 的表面粗粘 度, 获得了光学表面粗粘度和高度均匀的表面质量。

慢刀伺服切削是一种具有较高加工精度的切削 加工方法之一, 在光学加工中得到了广泛的应用。 CHEN 等 ${ }^{[64]}$ 建立了一种三维刀形补偿模型, 用于慢 刀伺服金刚石车削三维刀路的生成。HUANG 等 ${ }^{[65]}$ 从理论上确定了伺服动力学误差与形状误差之间的 关系, 为了实时补偿伺服系统的动力学误差, 提出 了一种协同伺服系统的概念, 即采用主从控制策略 将慢速伺服系统并入快速伺服系统。林泽钦等 ${ }^{[66]}$ 研 究了慢刀伺服加工微透镜阵列的刀具切削轨迹规划 过程, 考虑加工参数、刀具几何参数、透镜阵列几 何参数, 以及刀具与工件在三维方向的振动等因素 的影响, 提出微透镜阵列慢刀伺服加工的表面预测 模型。李荣涁等 ${ }^{[67]}$ 基于慢刀伺服车削技术, 对光学 组件进行加工, 使用正交慢刀伺服车削的方法加工 出波浪形微结构表面。HUANG 等 ${ }^{[68]}$ 提出了一种将 变主轴转速应用于 STS 的车削加工方法, 以减少成 形误差。为了解决微透镜在切削部位沿切削方向有 明显的颤振痕迹, LIU 等 ${ }^{[69]}$ 提出了一种基于 STS 的 铝合金(6061)滚子模具 MLAs 成形新方法, 与传统 的基于 STS 的加工方法相比, 这是一种高效的加工 方法。YAN 等 ${ }^{[70}$ 提出了一种基于慢刀伺服的圆角端 砂轮法向磨削方法, 保证了砂轮误差不传递到模具 表面, 从而达到了要求的加工精度。KONG 等 ${ }^{[71]}$ 研究了复合自由曲面的超精密加工及其表征, 提出 了一种慢刀伺服和快刀伺服相结合的复合自由曲面 加工方法。

\section{2 超精密磨削技术}

超精密磨削技术作为先进光学加工的技术手段 之一, 在该领域具有极为重要的地位。本节主要介 绍光学组件超精密磨削的几种方法——斜轴磨削、 超声振动磨削以及少轴弧面磨削, 主要对这些加工 方法在近十年的新进展进行综述。最后, 对厦门大 学智能制造与精密工程实验室团队在大口径光学非 球面超精密磨削机床研制方面的工作进行介绍。

\section{1 斜轴磨削}

通常情况下, 根据被磨削材料的不同结构形式， 所选用的磨削方法也会有所区别。如图 10 所示, 对 于传统光学非球面加工, 根据砂轮轴与工件回转轴之 间的相对运动关系, 可将其分为平行磨削法、横向磨 削法和斜轴磨削法, 其中, 斜轴磨削法根据砂轮与工 件的接触状态和运动方式又可以分为球面砂轮磨削 法、点磨削加工法、法向磨削法和展成磨削法。

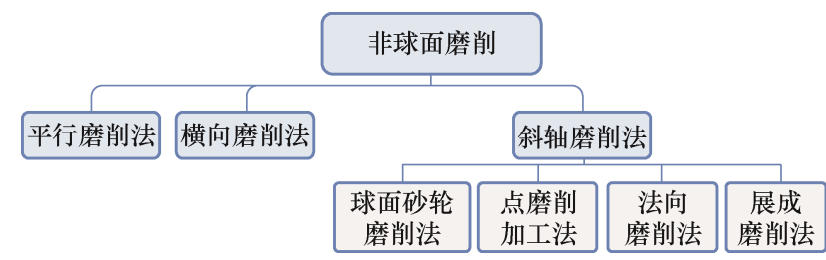

图 10 非球面磨削方法

平行磨削法是由庄司克雄 ${ }^{[72]} 、 S A E K I$ 等 ${ }^{[73]}$ 提出的 一种超精密磨削方法, 如图 11 所示 ${ }^{[74]}$, 国内众多学者 也对其进行了研究, 如厦门大学 WANG 等 ${ }^{[75]}$ 。采 用平行磨削法可以延长砂轮的使用寿命, 但是需要 对球头砂轮精密修整, 而球头砂轮的精密修整非常 困难。另外, 在磨削深径比大的工件时, 砂轮的工 件易干涉。横向磨削法是一种比较常规的磨削方法, 其广泛应用于大口径非球面透镜和光学模具碳化铇 和碳化硅等, 其示意图如图 12 所示 ${ }^{[76]}$ 。横向磨削 法适合于凸面加工, 安装使用方便, 刀具轨迹比较 简单, 只需要两轴联动。但是对砂轮直径限制比较 大, 特别是在凹面加工中砂轮的直径须小于工件被 加工点最小曲率半径。

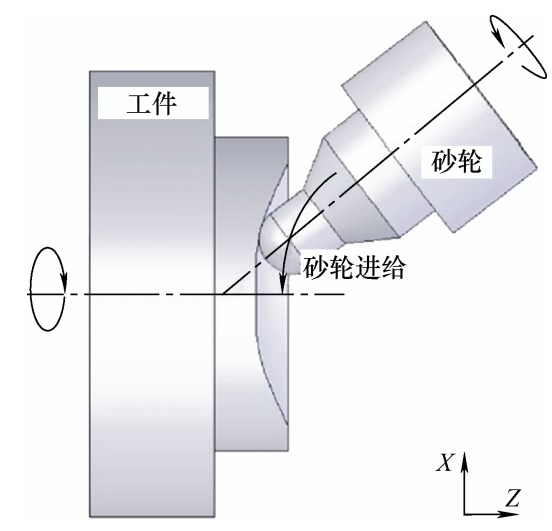

图 11 平行磨削法示意图

因此, 平行磨削法和横向磨削法不适合小口径 非球面零件的加工。对于小口径非球面零件, 一般 采用斜轴磨削法加工 ${ }^{[77]}$ 。斜轴磨削法根据砂轮与工 件的接触状态和运动方式又可以分为球面砂轮磨削 法、点磨削加工法、法向磨削法和展成磨削法, 本 文不对其进行展开综述, 仅就斜轴磨削这一大类方 


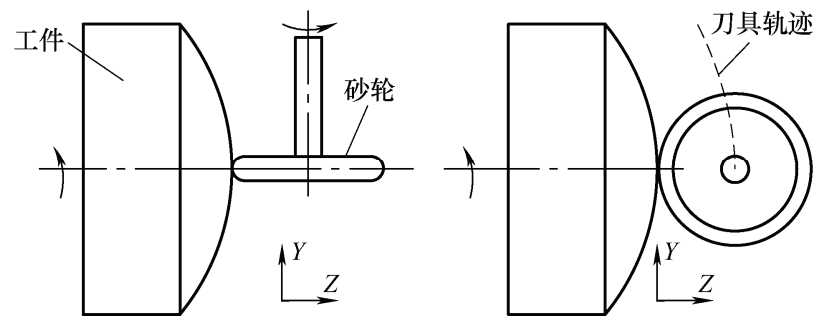

图 12 横向磨削法示意图

法进行综述。斜轴磨削法如图 13 所示 ${ }^{[78]}$ 。加工时砂轮 轴线与工件轴线同时位于同一平面内并呈一定夹角, 避免传统直交轴磨削法中砂轮轴与工件发生干涉的问 题。此外, 在使用斜轴磨削技术加工时, 砂轮沿非球 面路径移动同时绕砂轮轴线高速旋转, 而工件则绕机 床主轴低速旋转, 磨粒的切削方向与工件的旋转方向 保持平行, 斜轴磨削加工后工件表面粗糙度也优于 传统直交轴磨削法获得的表面 ${ }^{[79-80]}$ 。

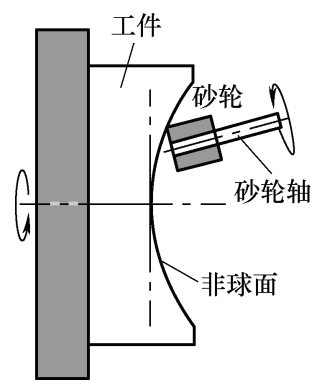

图 13 斜轴磨削法

1998 年, 日本的 SUZUKI 等 ${ }^{[81]}$ 首先提出了斜轴 磨削这一加工方法。2011 年, 王泽彪 ${ }^{[82]}$ 通过对微小 非球面磨削方式的总结和分析后, 采用 $X 、 Z 、 B$ 轴 三轴联动方式斜轴磨削, 进行微小非球面的磨削试 验, 并对该种磨削方式的加工干涉情况进行预测和 分析, 建立 $X 、 Z 、 B$ 轴三轴联动方式斜轴磨削加 工非球面的数学模型, 分析实际加工过程中的干涉 情况, 通过编程、计算获得不同非球面基圆半径和 非球面形状系数情况下, 磨削设定非球面的最大砂 轮直径和最大砂轮长度。

以获得高的加工效率和良好的表面质量。2012 年, 刘林枝 ${ }^{[83}$ 探讨了斜轴磨削小口径非球面时砂轮 的磨损测试方式与磨损量的变化规律, 研究了砂轮 半径误差对工件形状精度的影响。并在超精密磨床 上利用圆柱形砂轮对口径为 $6 \mathrm{~mm}$ 的超硬碳化铇的 凹形非球面模具进行超精密单点斜轴磨削试验, 并 对砂轮磨损误差进行了补偿。补偿后, 口径为 $6 \mathrm{~mm}$ 的非球面形状精度由 $P V 1929 \mathrm{~nm}$ 变为 $P V$ $359 \mathrm{~nm}$ 。2013 年, 陈逢军等 ${ }^{[84]}$ 针对 $\phi 2 \mathrm{~mm}$ 小口径 非球面光学透镜模具的斜轴磨削与误差补偿进行研 究。提出小口径非球面误差分析及补偿策略, 并分
析了砂轮 $x$ 方向的对心误差对形状精度的影响, 通 过提取法向残余误差的方法对加工后的随机形状误 差进行补偿。利用超精密磨床对口径为 $2 \mathrm{~mm}$ 的非 球面超硬碳化铇光学模具进行纳米斜轴误差补偿磨 削试验, 经过三次超精密磨削及误差补偿循环后, 光学模具的形状精度 $P V$ 从 $1034 \mathrm{~nm}$ 改善至 $146 \mathrm{~nm}$, 表面粗粘度达到 $R a 2.19 \mathrm{~nm}$ 。2016 年, 王 永强等 ${ }^{[79]}$ 选用碳化铇和不锈钢材料作为工件, 采用 斜轴磨削法, 对小口径非球面光学模具磨削加工进 行试验研究, 分析了工件转速、磨削深度、砂轮转 速、进给速度及砂轮粒度等参数对两种不同材料模 具的表面质量影响, 并得到磨削后表面质量随工件 转速、砂轮转速及砂轮粒度增加而变好, 随磨削深 度及进给速度增加而变差的结论。最终, 得到碳化 铇模具获得的表面粗糙度和面形精度分别为 $R a$ $1 \mathrm{~nm}$ 和 $P V 182 \mathrm{~nm} 。 2018$ 年, 尹韶辉等 ${ }^{[85]}$ 针对经 过超精密磨削后, 可以获得较高的形状精度, 但是 在后续的抛光过程中, 由于加工空间狭小，难以实 现自动化拋光的问题, 提出一种结合斜轴超精密磨 削和斜轴磁流变抛光的组合加工方法。并研制新型 的小口径非球面超精密复合加工机床, 对直径 $\phi 6.6 \mathrm{~mm}$ 的非球面碳化铇模具进行了加工试验。斜 轴磨削后加工表面粗粘度达到 $R a 6.8 \mathrm{~nm}$, 斜轴磁 流变抛光后表面粗䊁度达到 $R a 0.7 \mathrm{~nm}$, 面型精度可 以达到 $P V 221 \mathrm{~nm}$ 。

\section{2 超声振动磨削}

超声振动磨削是一种复合加工技术。由于加工 过程中引入了高频振动效应, 使得超声振动磨削加 工中磨粒的运动学特性及材料去除机制与普通加工 有很大区别。超声振动作用在提升加工效率、减小 切削力和切削温度、易于排屑和冷却润滑等方面有 着明显的技术优势 ${ }^{[86-90]}$ 。此外, 在超声振动辅助磨 削中, 由于高频振动的引入, 改变了加工过程中磨 具的运动学特性, 这将直接影响材料去除过程中裂纹 的生成与扩展、以及材料去除方式发生转变的临界载 荷和临界切削条件，其有利于降低裂纹缺陷提升塑性 去除能力。超声振动引发的裂纹阻抑作用可降低加工 中的中位裂纹和侧向裂纹的扩展程度，同时还可以降 低普通加工中单个裂纹引发脆性断裂的概率，对表面 微裂纹的扩展行为造成一定程度的干扰。超声振动作 用有助于减小亚表面侧向裂纹尺寸，降低侧向裂纹引 发的单扇形片状脆性剥落的断裂程度。

图 14 为超声振动磨削的加工原理。砂轮磨具在 主轴回转运动, 轴向超声振动和横向进给运动的复 合作用下对工件表面进行撞击并切削。在超声振动 
作用下, 砂轮和工件处于接触-分离-再接触到循环 状态之中, 因此冷却液可以顺利流进加工区并带走磨 削产生的热量和切屑。所以超声振动磨削技术在降低 磨削温度和排屑等方面具有一定优势 ${ }^{[91]}$ 。本文主要介 绍超声振动磨削技术在先进光学制造中的应用, 大致 分为超声振动磨削的理论模型建立、相关的工艺探索 以及超声振动磨削相关的加工体系设计。

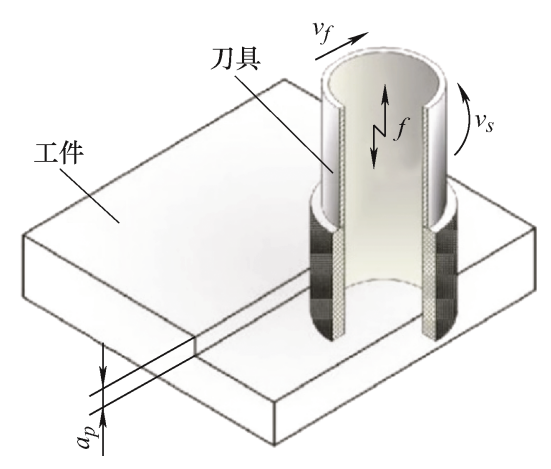

图 14 超声振动磨削加工原理

首先是关于超声振动磨削理论模型的建立过 程。2016 年, ZHOU 等 ${ }^{[2]}$ 研究了超声振动参数、磨 削参数和工作材料固有特性对去除脆性模态和韧性 模态比能的影响。根据玻璃在延性和脆性加工模式 下的切削能量消耗的解析表达式, 提出了一种预测 玻璃在超声振动辅助磨削中延性-脆性转变临界切 削深度的方法, 建立了超声振动辅助玻璃材料加工 中临界切削深度与比切削能量的预测模型。临界切 削深度在延性和脆性模式下比能量消耗曲线的交点 处确定。对 BK7 和 JGS1 光学眼镜进行了刮擦试验, BK7 和 JGS1 玻璃的临界切削深度预测值分别为 $2.13 \mu \mathrm{m}$ 和 $2.79 \mu \mathrm{m}$, 试验值分别为 $2.2 \mu \mathrm{m}$ 和 2.88 $\mu \mathrm{m}$, 验证了模型的有效性。2017 年, 赵培轶 ${ }^{[91]}$ 基 于塑性去除模式和脆性去除模式的切削比能随切削 深度变化的计算分析, 建立了 BK7 光学玻璃脆-塑 性转变临界切削深度的预测模型, 并进行了相关试 验验证, 为后续加工表面质量及亚表面裂纹损伤的 研究与分析提供了理论依据。同时, 建立了超声振 动磨削 BK7 光学玻璃加工表面粗糙度的预测模型, 并通过试验验证了该模型的正确性, 建立了亚表面 裂纹损伤最大深度的预测模型, 为探寻减免亚表面裂 纹损伤的工艺措施提供了依据。2019 年, 孙国燕 ${ }^{[93]}$ 以低膨胀玻璃 Zerodur 和 ULE 为研究对象, 开展了 微纳米压痕试验、普通刻划和轴向振动刻划试验, 计算了材料脆塑本征性能, 观察了材料在单磨粒作 用下的动态去除过程和去除方式, 并重点分析了单 磨粒刻划中的轴向超声振动、脆塑去除模式以及材 料性能对材料去除机理的影响作用, 最终建立了表
征磨削质量指标的磨削力、 $R a$ 和亚表层裂纹最大深 度预测模型相结合为综合磨削质量预测模型, 在对 磨削质量的高效预测基础上与材料去除率结合，探 索出实现高质、高效和低损伤的超声振动辅助圆周 磨削低膨胀光学玻璃的最优工艺路线。这为超声振 动辅助圆周磨削的工艺优化奠定了理论基础, 更对 实现低膨胀玻璃光学组件的大批量、高质量、短周 期和低成本加工具有重要意义。

其次是对超声振动磨削相关工艺探索的综述。 2012 年, 郭兵 ${ }^{[94]}$ 研制了适用于超硬微结构光学菜单 面的超声振动磨削系统, 揭示了超硬微结构表面的 超声振动磨削加工机理, 分析了金刚石砂轮在加工 微结构表面时的磨削性能演化规律。同时研究了超 声振动参数、磨削工艺参数以及表面结构参数对超 硬微结构光学菜单面完整性的影响规律, 并在此基 础上优化了超声振动磨削工艺方法, 进而解决了现 有超硬材料微结构光学菜单面超精密磨削加工中存 在的关键问题。同时还进行了典型超硬微结构光学 菜单面的超声振动磨削加工工艺研究。加工对象为 无结合剂碳化铇、碳化硅陶瓷和氮化硅陶瓷等超硬 模具材料, 具体微结构表面包括微圆弧槽阵列、闪 耀光栅、金字塔矩阵以及阶梯光栅。2019 年, JIANG 等 ${ }^{[95]}$ 研究了光学玻璃超声振动磨削的比磨削能量和亚 表面损伤深度, 以估算在光学玻璃制造中使用超声振 动磨削所增加的能量和节省的时间。结果表明, 超声 振动磨削可降低玻璃磨削过程中的能耗, 并可显着节 省后续抛光过程中的时间。因此, 超声振动磨削工艺 在绿色制造光学组件方面显示出了良好的应用潜力。

2019 年, 许陆昕 ${ }^{[96]}$ 针对碳化硅陶瓷磨削时存在的问 题, 进行了碳化硅陶瓷超声振动磨削表面质量的研究, 其进行了超声振动磨削的运动学分析, 研究了超声振 动磨削的表面创成机理, 结果发现超声振动磨削非常 适合应用于小直径砂轮的磨削。超声振动磨削可以有 效地解决小直径砂轮磨削碳化硅时存在的问题, 提高 表面质量和磨削效率。

最后, 是超声振动磨削相关的加工体系设计。 2018 年, 周莲 ${ }^{[97]}$ 确定了 $\mathrm{SiC}$ 零件超声振动磨削系统 的组成部分及功能要求, 设计了超声振动磨削主轴 的结构; 并根据全波长超声换能器理论, 计算了振 子结构; 依据电磁感应原理设计了一种考虑分布电 容和漏感影响的新型非接触电信号传输装置, 解决 了传统接触式电信号传输方式存在的摩擦大、发热 严重、振动大和转速低的问题。

\section{3 少轴弧面磨削}

少轴弧面磨削通常采用倾斜弧面砂轮, 通过控 
制砂轮的运动轨迹以及磨削点在砂轮弧面上的移 动, 保证运动过程中磨削点处砂轮表面与工件曲面 法向量一致, 最终由运动包络的形式形成目标曲面。 少轴弧面磨削中的两个虚拟轴通过磨削点在砂轮曲 面上的移动形成。与传统上使用的五轴非球面光学 镜面磨削相比, 少轴弧面磨削通过减少机床运动轴 数提高了系统刚度, 通过磨削点在砂轮表面上的移 动均化了砂轮磨损 ${ }^{[98]}$ 。图 15 为传统五轴磨削示意 图, 图 16 为少轴弧面磨削示意图 ${ }^{[98]}$ 。

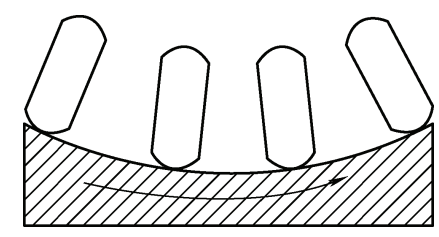

图 15 传统五轴磨削

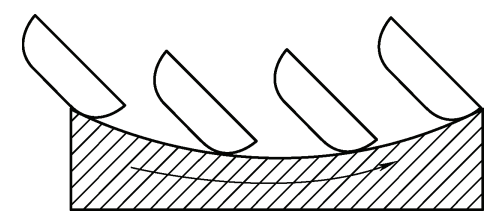

图 16 少轴弧面磨削

林硕 ${ }^{[99}$ 基于 $\mathrm{T} 3$ 构型机床弧面包络法磨削下的加 工路径规划方法与三维砂轮-工件接触面的计算方法,
建立了非球面面形误差预测及相应的补偿算法, 主 要包含了砂轮几何参数识别误差、砂轮轴倾角误差、 砂轮轮廓误差与砂轮轮廓磨损。以口径 $600 \mathrm{~mm}$-近 轴曲率半径 $1500 \mathrm{~mm}$ 的碳化硅非球面进行面形精 度分析, 确定砂轮轮廓误差与砂轮轮廓磨损为主要误 差来源。结合非球面面形误差测量与三轴联动法向补 偿, 实现了有效口径 $80 \mathrm{~mm}$-近轴曲率半径 $325 \mathrm{~mm}$ 的 碳化硅抛物面与有效口径 $580 \mathrm{~mm}$-近轴曲率半径 $50 \mathrm{~m}$ 的石英玻璃抛物面的补偿加工。前者 RMS 值由 $1.44 \mu \mathrm{m}$ 下降至 $0.9 \mu \mathrm{m}$, 误差下降率达 37\%；后者 RMS 值由 $13.7 \mu \mathrm{m}$ 下降至 $1.8 \mu \mathrm{m}$, 误差下降率达 $86 \%$ 。

\section{4 超精密磨削机床 UPG80 研制介绍}

厦门大学智能制造与精密工程实验室课题组承担 “高档数控机床与基础制造装备” 科技重大专项子课题 “大口径光学非球面超精密磨削机床研制”，面向磨削 组件最大尺寸 $1300 \mathrm{~mm} \times 750 \mathrm{~mm} \times 550 \mathrm{~mm}$, 其中要求 $530 \mathrm{~mm} \times 530 \mathrm{~mm}$ 非球面面形精度 $P V \leqslant 5 \mu \mathrm{m}$ 。

图 17 为超精密磨削机床 UPG80 整机外观及结 构图, 大口径光学非球面超精密磨削机床整机为立 柱移动式数控卧轴矩台平面磨床结构, 整体采用模 块化结构。 $X 、 Y 、 Z$ 三直线轴均采用拼块式液体静 压导轨结构, 其中, $X$ 直线轴结构如图 18 所示。砂 轮旋转主轴采用恒压式液体静压技术。

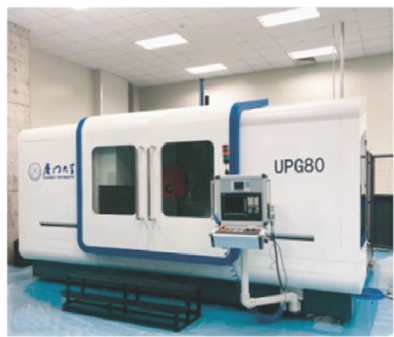

(a)

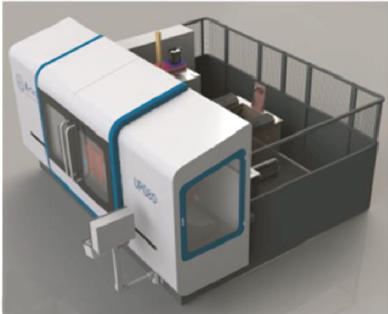

(b)

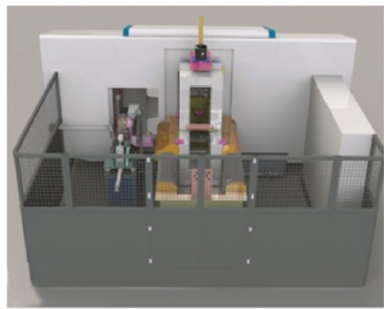

(c)

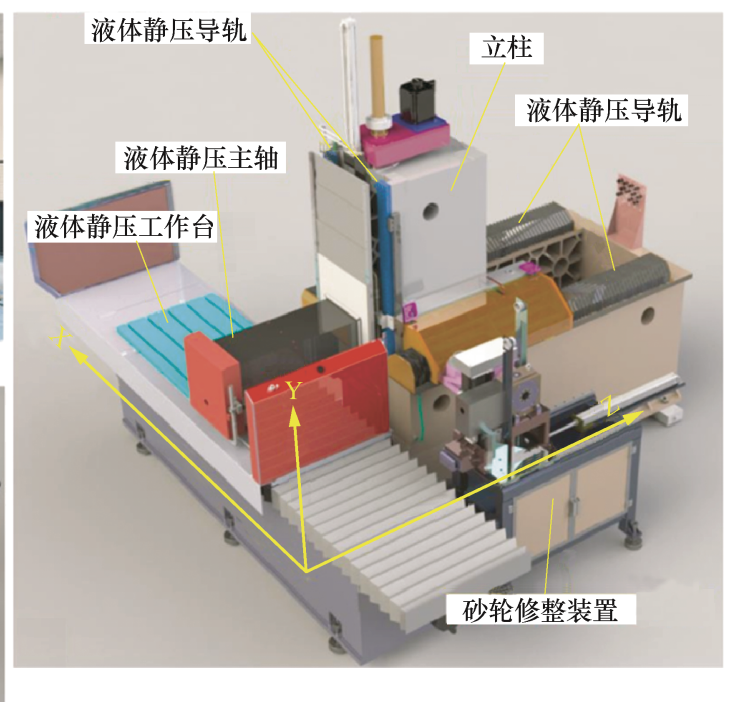

(f)

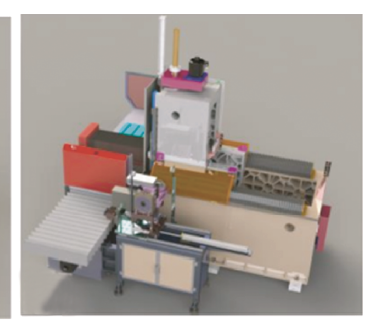

(e)

图 17 超精密磨削机床 UPG80 整机外观及结构图 


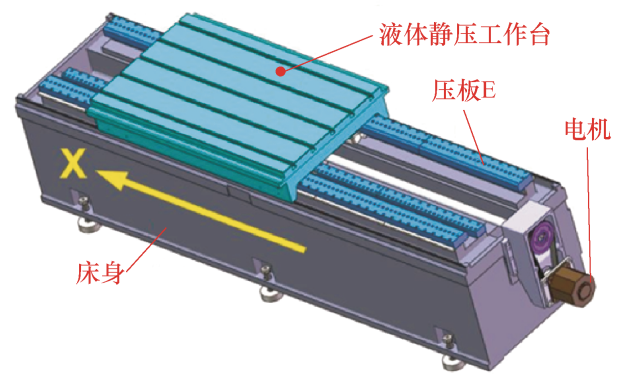

(a) 六滑块静压工作台系统

HGS：液体静压滑块

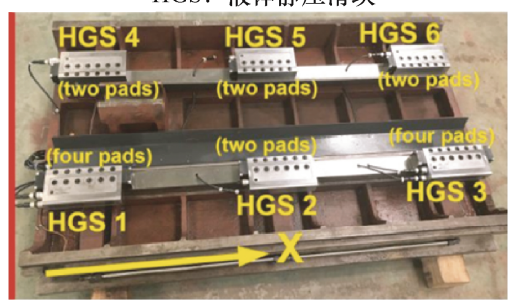

(c) 六滑块分布

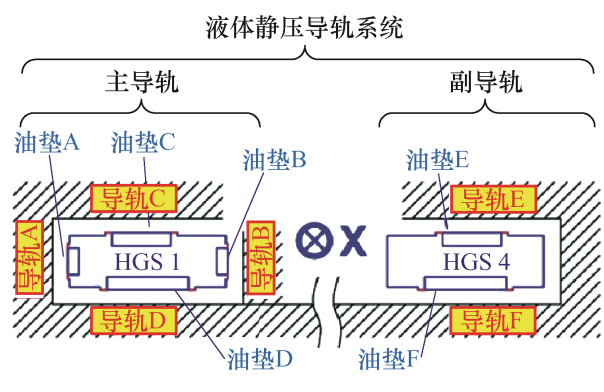

(b) $X$ 轴向视图

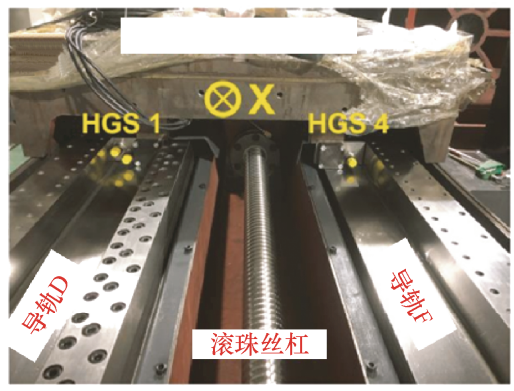

(d) 开式装配

图 18 超精密磨削机床 UPG80 液体静压工作台拼块式结构

工艺验证试验表明, 使用半精磨砂轮进行大平 面反射镜磨削试验: $1300 \mathrm{~mm} \times 750 \mathrm{~mm}$, 全口径检 测面形误差, 工件面形误差 $P V$ 值约 $7.5 \mu \mathrm{m}$, 如图 19 所示; 精磨 $530 \mathrm{~mm} \times 530 \mathrm{~mm}$ 口径非球面光学 组件, 工件面形误差 $P V$ 值约 $P V 3.0 \mu \mathrm{m}$, 如图 20 所示。

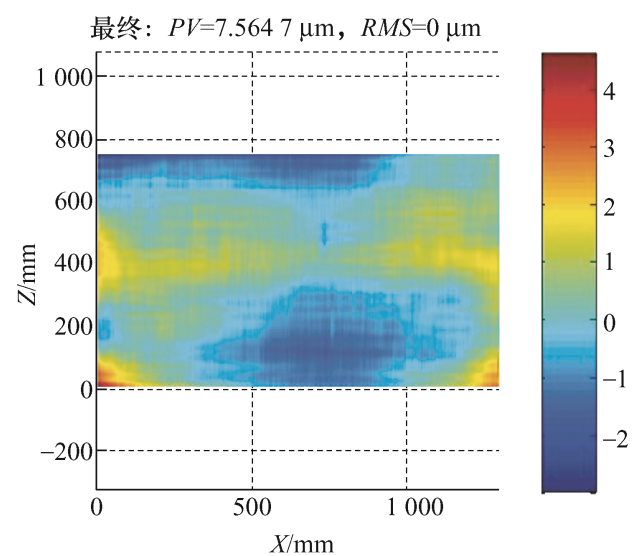

图 19 全口径 $1300 \mathrm{~mm} \times 750 \mathrm{~mm}$ 面形误差检测结果

本节主要介绍了斜轴磨削、超声振动磨削以及 少轴弧面磨削这几种超精密磨削方法, 并对其行了 分析和对比。与传统的平行磨削法和横向磨削法相 比, 斜轴磨削法通过使砂轮轴线与工件轴线同时位 于同一平面内并呈一定的夹角, 避免了砂轮轴与工 件发生干涉的问题; 此外, 由于加工过程中磨粒的 切削方向与工件旋转方向保持平行, 斜轴磨削加工 后的表面粗粘度也优于传统的直交轴磨削法获得的 表面。超声振动磨削法是一种复合加工技术, 由于
高频振动效应的引入使得加工中的磨粒运动学特性 以及材料去除机制与普通的磨削有很大的区别; 同 时，这直接影响了材料去除过程中裂纹的生成与扩 展, 有利于降低裂纹缺陷提高塑性去除能力; 此外, 超声振动磨削技术在降低磨削温度和排屑等方面也 具有一定的优势。与传统的五轴非球面光学镜面磨 削相比, 少轴弧面磨削通过减少机床运动轴数提高 了系统的刚度, 并通过磨削点在砂轮表面上的移动 均化了砂轮磨损。

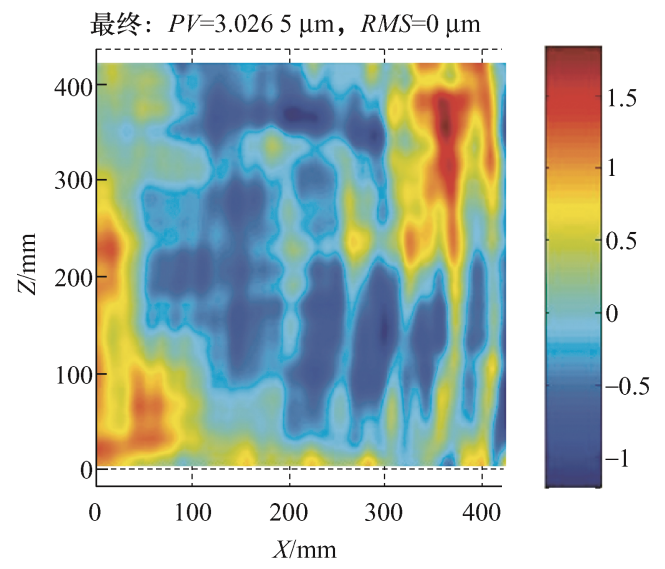

图 20 口径 $530 \mathrm{~mm} \times 530 \mathrm{~mm}$ 非球面面形误差检测结果

\section{3 超精密抛光技术}

超精密抛光技术一般是指通过微磨粒或物理化学 的方法使工件表面粗糙度达到纳米级甚至亚纳米级的 表面精加工技术 ${ }^{[100]}$ 。一般来说, 超精密抛光是车削 
或磨削工艺之后的加工链的最后一道工序 ${ }^{[101]}$, 其主 要目的是减少工件残余表面形貌和亚表面层, 去除 先前的工艺缺陷, 提高表面粗粮度从而获得光滑洁 净的表面 ${ }^{[100]}$ 。实现超精密抛光的方法有很多种, 传 统的方法有化学机械抛光、气囊抛光等, 这些方法 在加工过程中由于方法本身的原因需要与工件直接 接触, 不可避免的会造成表面损伤。一些非接触的 抛光方法如等离子体抛光, 离子束抛光等由于其方 法本身的优势, 不与工件直接接触, 所以在获得少 或者无表面/亚表面损伤的表面方面具有较大的优 势, 但是其加工效率往往较低。一些新的非接触的 方法如剪切增稠抛光等同时具备非接触和较高的抛 光效率, 在光学制造领域具有较大的潜力。本节主 要介绍并归纳了近十年来光学制造领域拋光方法的 新进展, 及出现的一些新的抛光方法。

\section{1 离子束抛光}

离子束抛光技术在上世纪末期由 WILSON 和 REICHER 等提出, 是一种原子量级上的无应力、非 接触式的先进抛光工艺, 抛光过程由计算机控制, 加工过程确定性高, 面型收玫快, 还具有加工精度 高, 被加工镜面洁净无污染等特点, 可加工材料广 泛，通常有金属、陶瓷、宝石、316L 不锈钢、AZI 镁合金、高温金属、高速钢和复合氮化物硬质涂层 等, 特别适合非球面, 正六边形镜面等不规则镜面 的加工。但也存在抛光效率低下、装置成本高、控 制复杂等缺点。针对这些局限性, 近十年来研究工 作者们主要在物理模型及数值模拟研究, 加工参数 影响和装置改进开发三方面做了较多的探索工作, 如图 21 所示。

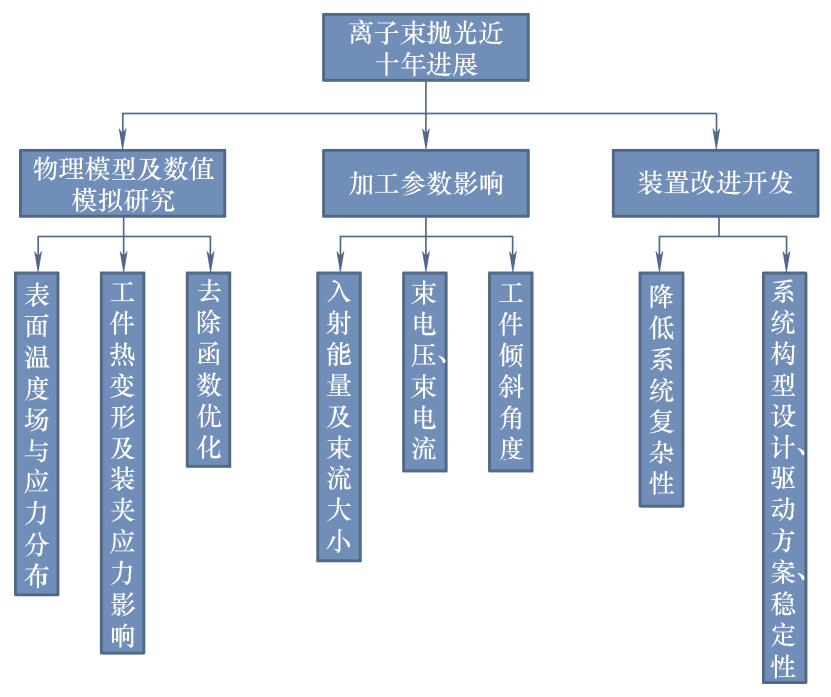

图 21 离子束抛光近十年研究进展

离子束抛光 ${ }^{[102-104]}$ 是基于原子溅射理论而发展 起来的一种去除精度达原子级别的抛光技术, 带有
一定能量的离子束流轰击工件表面后, 与浅层光学 表面内的原子不断碰撞进行能量交换, 获得能量的 原子继续将能量传递给周围原子, 形成级联运动。 当原子由于碰撞所获得的能量大到可以挣脱表面束 缚时, 将从固体表面脱离出去形成溅射原子, 以此 达到原子级材料去除。

离子束抛光的热效应对材料的加工具有巨大的 影响, 随着加工温度变化产生的热应力对工件的加 工质量会有一定影响, 徐明进等 ${ }^{[105]}$ 采用有限元分析 软件 ANSYS 模拟了离子束加工 KDP 晶体表面的温 度场和应力场分布, 发现了离子束加工受热区产生 拉应力和压应力。由于离子束加工过程中热应力的 变化是沿着离子束扫描的径向方向进行, 所以工件 表面所受的径向压力最大, 周向压力次之, 轴向压 力最小。由于离子束加工过程中温度较高, 由此产 生的热变形能否恢复也是一个值得探究的问题。张 云飞等 ${ }^{[106]}$ 利用 ANSYS 软件对工件热变形进行仿真 分析, 并与实际测量进行比较, 结果显示, 离子束 在抛光过程中工件的温度可升高至 $200{ }^{\circ} \mathrm{C}$, 热变形 能否恢复的影响因素是装夹应力。除了数值模拟研 究, 对于去除函数的理论模型优化也有一定发展, 唐瓦等 ${ }^{[107]}$ 针对三轴离子束系统抛光大口径高陡度 离轴非球面过程中镜面曲率变化进行研究, 提出了 去除函数修正矩阵对离子束抛光去除函数进行优 化, 并通过试验对去除函数进行验证修正误差小 于 $1.5 \%$ 。

在离子束加工过程中, 针对不同材料, 不同的 工艺参数对加工质量影响可能不同。针对离子束加 工其主要作用的离子束能量、束电压和束电流以及 入射角度四个参数, 主要有以下三方面发现: 张厚 亮等 ${ }^{[108]}$ 采用不同入射能量的 $\mathrm{Ar}^{+}$离子束溅射倾角为 $30^{\circ}$ 的 U 薄膜表面。结果发现, 随溅射时间的延长 入射能量为 $1.0 \mathrm{keV}$ 的 $\mathrm{Ar}^{+}$离子束, 表面粗䊁度逐渐 增大; 而采用 $0.5 \mathrm{keV}$ 的 $\mathrm{Ar}^{+}$离子束, 粗䊁度值趋于 减小。表明入射能量对工件的抛光效果有着决定性 的影响; 另外, 束电压和束电流的大小对工件表面 粗糙度也可能会有一定影响, 冯殊瑞等 ${ }^{[109]}$ 研究了束 电压和束电流的大小对易潮解的 KDP 晶体表面粗 䊁度的影响, 结果显示, 随着束电压的增大, 晶体 表面粗糙度值变大, 而束电流对晶体粗粘度的影响 相对较小。但随着去除深度的增加, 晶体表面在车 削后残留的亚表面缺陷层就会暴露出来, 使得晶体 表面粗粘度值变大; 除以上因素外, 由于不同的入 
射角加工出的工件表面质量也有一定区别, 武否等 ${ }^{[110]}$ 采用微波离子源对 $\mathrm{ZnS}$ 进行抛光处理, 研究了离子 束入射角度对工件刻蚀速率以及表面粗糙度的影 响, 最终得出采用 $400 \mathrm{eV}$ 的离子束能、 $35 \mathrm{~mA}$ 的离 子束流以及 $45^{\circ}$ 的入射角度时, $\mathrm{ZnS}$ 表面粗粘度有 最大降幅 $0.23 \mathrm{~nm}$ 离子束垂直入射抛光较难改善表 面粗糙度, 倾斜入射抛光可以较好地改善表面粗糙 度, 入射角为 $30^{\circ} \sim 60^{\circ}$ 之间时抛光效果最佳, 表 面粗糙度得到明显改善; 倾斜 $45^{\circ}$ 入射均匀去除抛 光试验结果表明表面粗楉度的 RMS 值由抛光前 $(0.92 \pm 0.06) \mathrm{nm}$ 下降到 $(0.48 \pm 0.04) \mathrm{nm}$, 提高了光 学零件的表面质量。

尽管离子束抛光目前的研究已经相当成熟, 但 是传统的离子束抛光装置昂贵, 系统复杂, 不适用 于大规模工程应用。近几年, 在对离子束抛光装置 进一步的开发方面, 在系统简化和增强控制方案稳 定性方面做了一定的工作。DEMMLER 等 ${ }^{[111]}$ 开发 了三轴运动系统离子束抛光装置, $X$ 轴和 $Y$ 轴用于 面扫描, $Z$ 轴用于光学组件的表面轮廓的焦点调整。 减少两个额外的倾斜轴用于入射角调节, 巧妙地降 低了系统的复杂性。试验结果表明, 可以将球面或 非球面的误差修正到 $\lambda / 50(12.64 \mathrm{~nm})$ 及以下。尹小 林 ${ }^{[112]}$ 建立了基于混联构型离子束修形五轴运动系 统的构型设计及控制方案, 实现了从驱动杆到离子 源的运动学分析求解, 为保证加工过程中离子源运 动的稳定性, 对设计的并联机构的位形奇异性进行 了分析和验证。

近十年来离子束抛光技术的机理及试验方面 的探索已经较为成熟, 综上未来的离子束抛光更 应聚焦在以下几个方面, 争取更大的突破: (1) 获 取离子束抛光驻留时间矩阵之后, 如何更快速、更 高效、更高精度地划分轨迹段及其进给速度求解算 法; (2) 降低系统复杂性, 减少装置成本, 推广工程 应用; (3) 探索新的复合技术，提高抛光效率。

\section{2 等离子体抛光}

等离子体抛光由于其加工原理上的优势, 在抛 光过程中不存在机械力的作用, 从而可以避免机械 应力对材料造成的表面或亚表面损伤, 最终在实现 纳米级表面粗粘度的同时实现无损伤的表面。基于 这种优势, 等离子抛光方法也受到了研究学者们的 青睐并在近些年得到了一定的发展。等离子体抛光 在光学领域也有着较广泛的应用 ${ }^{[113]}$ 。图 22 为等离 子抛光示意图。

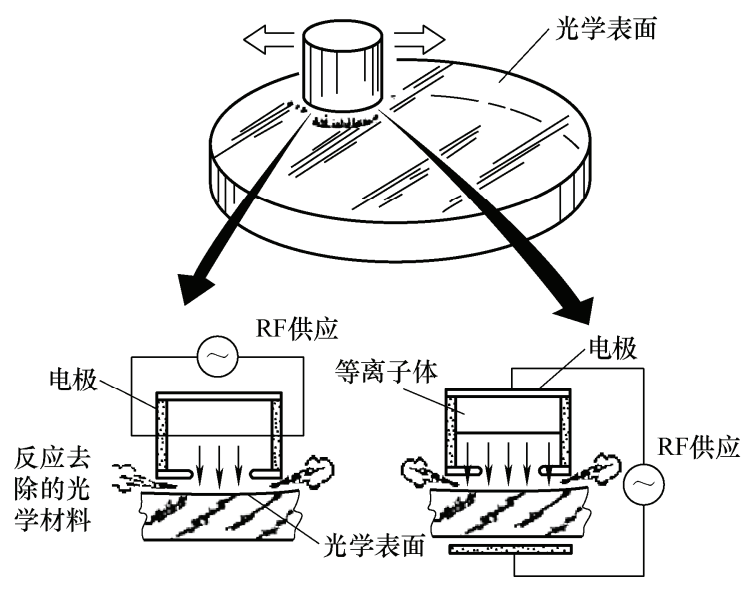

图 22 等离子抛光示意图

图 23 为等离子抛光的材料去除原理示意图。 等离子体抛光实质上是一种化学反应过程, 加工 过程中等离子体中的活性反应原子与工件表面 原子发生化学反应并产生具有较强挥发性的物 质(气态生成物)实现材料的去除, 且等离子抛光 不会引入表面污染物。另外, 采用等离子抛光方 法生成的表面一般不存在表面/亚表面损伤，但 加工效率一般, 可用于加工球面、非球面以及自 由曲面等。此外, 在等离子抛光过程中, 等离子 体通常由射频放电激发, 等离子在电场中的加速 时间较短使得等离子体的能量比较低, 所以等离 子体在轰击工件表面时晶格结构破坏微弱, 能够 获得光滑的表面。

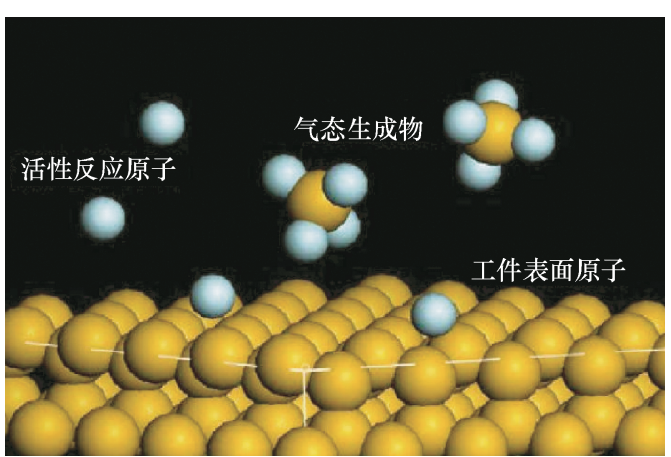

图 23 等离子抛光的材料去除原理示意图

建立等离子体抛光的仿真模型有助于了解 抛光过程中的材料去除过程, 进一步揭示工件表 面的演化过程。ZHANG 等 ${ }^{[114]}$ 建立了大气等离子 抛光的量子化学模型用于研究原子之间的相互 作用, 如图 24 所示。通过比较空间原子构型和 粒子扩散路径, 证明了材料凸起形貌比凹陷形貌 去除得更快。 


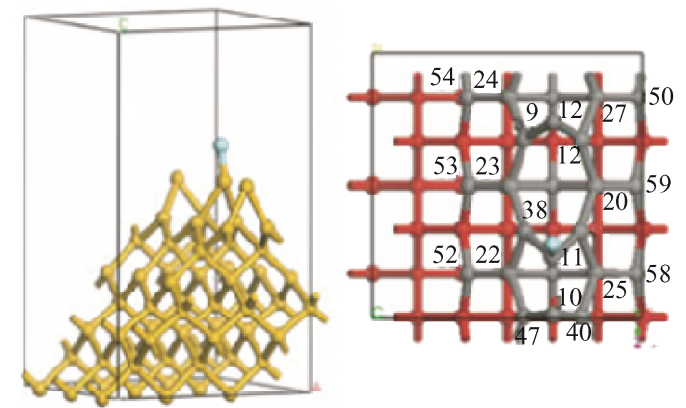

(a) 凸特征

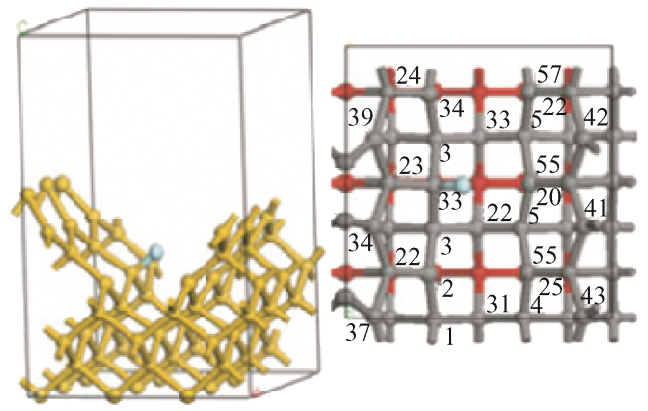

(b) 凹特征

图 24 具有 $\mathrm{Si}-\mathrm{F}$ 键的典型 $\mathrm{Si}(100)$ 表面形貌模型

基于等离子体抛光非接触的优势, 一些研究人 员在多种光学材料开展抛光试验并取得了较好的结 果。ARNOLD 等 ${ }^{[115]}$ 使用大气等离子体射流加工的 方法, 以非接触的方式加工 $\mathrm{SiC}$ 反射镜并取得了良 好的效果。加工后其表面从 $62.25 \mathrm{~nm} P V, 8.26 \mathrm{~nm}$

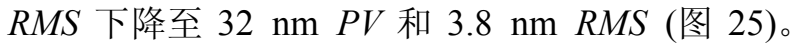
LALIOTIS 等 ${ }^{[16]}$ 研究了电感耦合等离子体抛光如何 改善光学微腔镜面基片的形状和粗䊁度。使用原子 力显微镜测得其表面粗糙度可达到 $1 \mathrm{~nm}$, 在涂覆高 反射率电介质后, 该表面粗糙度可降低至 $0.5 \mathrm{~nm}$ 。 CASTELLI 等 ${ }^{[117]}$ 使用反应原子等离子体抛光熔融 石英样品, 通过优化刀具轨迹算法与迭代过程计算 得到了更快收敛速度。迅速实现了图形误差修正至 $\lambda / 40 R M S$ 。在最多三次迭代中，获得了 $78 \%$ 至 $89 \%$ 之间的高总体收敛率证实了反应原子等离子体抛光 大型表面的潜力。针对对金刚石、碳化硅、蓝宝石 等难加工材料的抛光, DENG 等 ${ }^{[118]}$ 使用等离子辅助

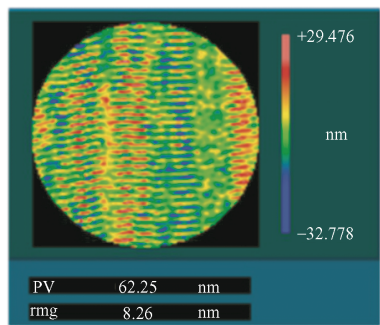

(a) 加工前

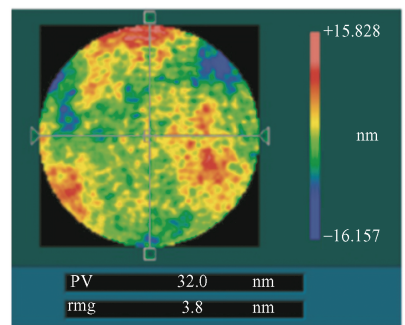

(b) 加工后
图 25 加工前后的 $\mathrm{SiC}$ 表面
抛光方法抛光了碳化硅材料。通过提高氧化速率和 降低抛光速率，获得了具有 $11.42 \mathrm{~nm} P V$ 和 $0.91 \mathrm{~nm}$ $R M S$ 的光滑表面。

由于等离子体抛光方法原理上的局限性, 该 方法虽然可以实现较高的表面质量，但在材料去 除率方面却相对较低。为了提高等离子体抛光的 材料去除效率, 伍艺龙 ${ }^{[113]}$ 使用等离子抛光方法加 工了石英样片, 试验结果可得添加氧气会对 $\mathrm{SF}_{6} / \mathrm{Ar}$ 等离子体的抛光效率产生影响, 当六氟化 硫与氧气的比例为 $2: 1$ 时, 抛光速率最高, 可达 $3.6 \mathrm{~nm} / \mathrm{min}$ (图 26)。WU 等 ${ }^{[119]}$ 在自制的等离子抛 光试验装置上进行多次试验。讨论了基片表面粗 糙度与不同工艺参数之间的关系。结果表明，在 氩等离子体中加入 $\mathrm{SF}_{6}$ 可减少氩原子的对工件表 面的轰击效应并使祄底平滑。在 $\mathrm{SF}_{6} / \mathrm{Ar}$ 等离子体 中适当添加 $\mathrm{O}_{2}$ 可提高抛光效果和去除效率。王东

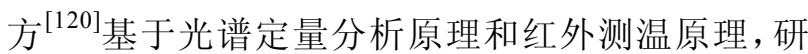
究了不同工艺参数对光学材料熔石英去除率的影 响规律。试验结果表明, 当 $\mathrm{CF}_{4}$ 的含量为 $3.5 \%$ 时, 熔石英的去除深度每分钟最高达 $3.5 \mu \mathrm{m}$, 加入适 量的 $\mathrm{O}_{2}$ 能进一步提高去除率。史宝鲁 ${ }^{[121]}$ 开发了 新的可提高加工效率的电弧增强等离子体加工方 法。基于 Arrhenius 公式, 建立了电弧增强等离 子体加工效率模型, 发现当电弧形成时, 化学反 应活化能降低, 使化学反应发生所需要的能量降 低, 从而提高了等离子体加工效率。PAETZELT 等 ${ }^{[122]}$ 开发了一种精细聚焦等离子体射流抛光方 法, 可用于对晶体硅等硅基材料的局部等离子体 辅助抛光。这种等离子喷射工具的典型工具功能 宽度为 $0.5 \sim 1.8 \mathrm{~mm}$ ，材料去除率高达 $0.068 \mathrm{~mm}^{3} / \mathrm{min}$ 。为了提高光学组件大气等离子体 加工的精度, 夏龙光 ${ }^{[123]}$ 针对连续位相板的设计面 形，提出了一种基于 Nyquist 定理的去除函数匹 配方法。分别使用点位加工模式和速度模式对 $320 \mathrm{~mm} \times 320 \mathrm{~mm} \times 2 \mathrm{~mm}$ 的连续位相板进行对比 抛光试验。相较于点位模式速度模式将加工时间 由 $13.2 \mathrm{~h}$ 减少至 $9.3 \mathrm{~h}$, 面形误差 $R M S$ 值由 $100 \mathrm{~nm}$ 降低至 $80 \mathrm{~nm}$, 具有一定的优越性。 $\mathrm{SU}$ 等 ${ }^{[124]}$ 提 出使用大气压等离子体和气囊抛光组成的组合加 工链, 其中大气等离子抛光起到生成面形的作用, 气囊抛光起到快速平滑表面的作用。试验结果表 明, 抛光诱导的面形变化小于 $20 \mathrm{~nm}$, 粗糙度提 高到 $2 \mathrm{~nm}$, 验证了组合加工链的可行性。 


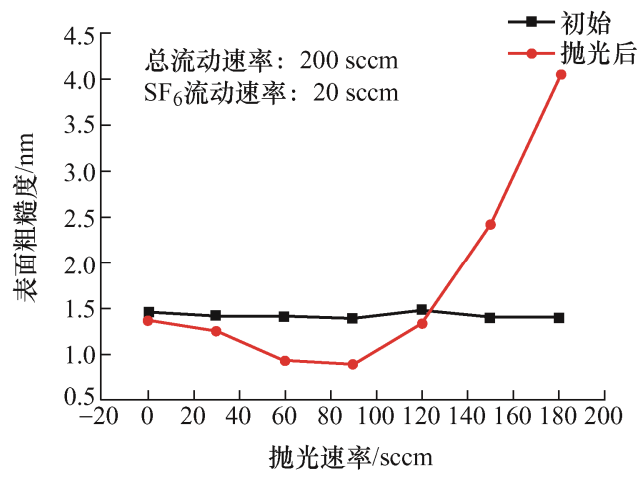

(a) 表面粗粘度

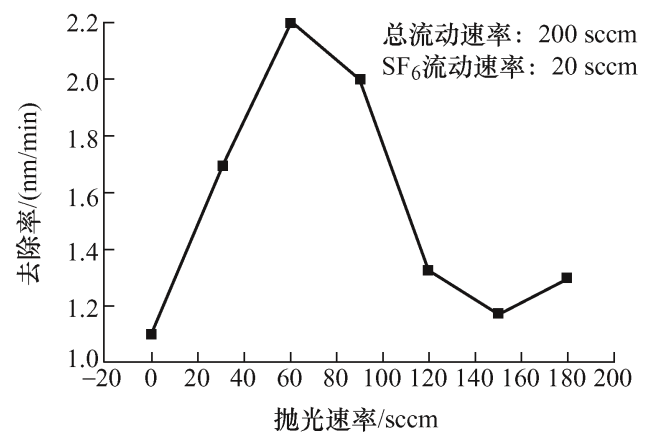

(b) 去除率

图 26 表面粗粘度及材料去除率随 $\mathrm{Ar}$ 气流动速率的变化

由于等离子体抛光需要较高的实现条件, 且需 要较多的外围辅助设备以及相应的闭环反馈控制系 统。为提高数控化学等离子体加工的空间分辨率并 节省设备成本, YAMAMURA 等 ${ }^{[125]}$ 开发了等离子体 脉宽调制控制单元，代替工作台的扫描速度控制，以 控制去除量分布(图 27)。在设定的去除深度急剧变化 的位置, 采用脉宽调制控制的图形去除误差急剧下降 到 4\%以下, 而采用工作台扫描速度控制的图形最大去 除误差为 $20 \%$ 。JOURDAIN 等 ${ }^{[126]}$ 开发了一种新的等 离子体抛光设备—太阳神 1200(图 28)。与其他等离 子体抛光设备不同, Helios 1200 在大气压下操作等离 子炬，提供高材料去除率和相对较低的运行成本。该 设备非常适合加工大型光学组件、轻质光学器件、硅 基和难以加工的材料、非球面和自由曲面。

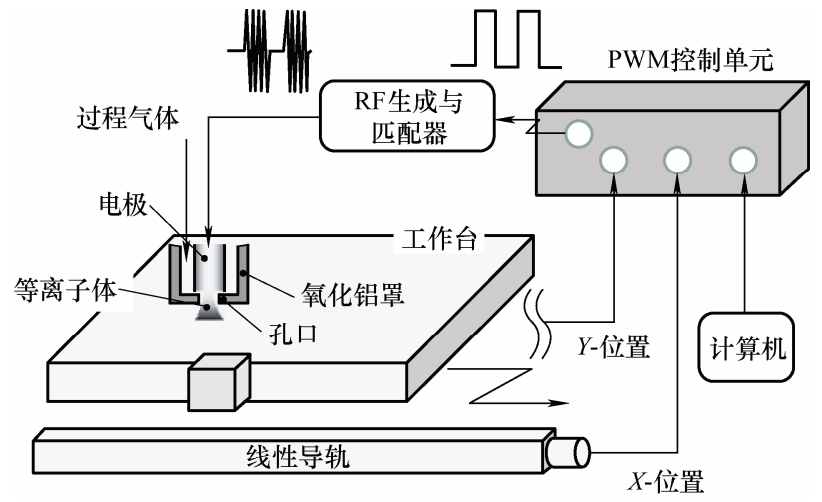

图 27 经脉冲宽度调制的化学等离子体加工系统

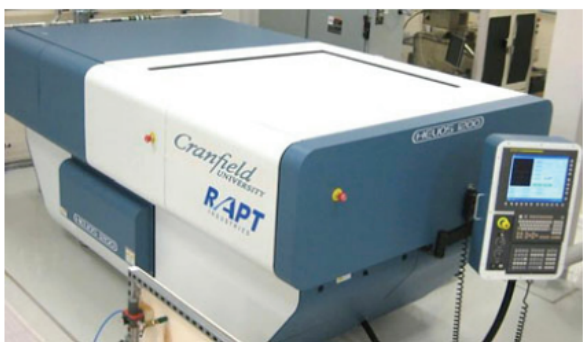

图 28 Helios 1200 等离子体抛光机

\section{3 气囊抛光}

气囊抛光技术(Bonnet polishing) 是英国伦敦大 学学院光学科学实验室(OSL)和 Zeeko 公司的 BINGHAM 等 ${ }^{[127]}$ 在 2000 年提出的一种柔性抛光技 术, 目前已被广泛应用到光学组件的超精密加工中。 这项技术的原理是通过驱动一个气压在线可控、表 面覆盖有抛光垫的柔性气囊头旋转完成对工件的抛 光加工。图 $29^{[128]}$ 是自由曲面的气囊抛光原理结构 示意图。由于气囊头柔性可控, 抛光工具与工件表 面接触时有较大的接触面积且能够良好贴合, 既保 证了抛光效率又提高了加工精度, 尤其适用于非球 面、自由曲面的加工。

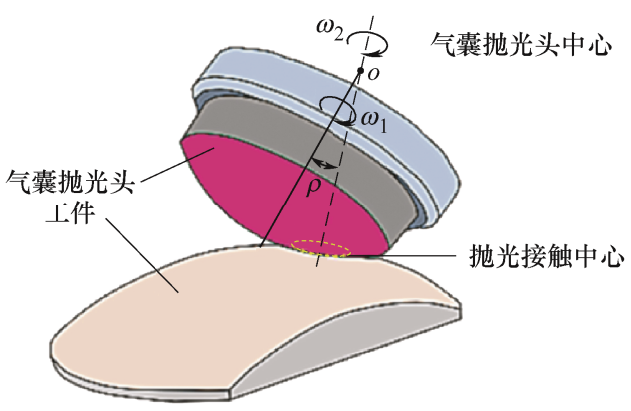

图 29 自由曲面的气囊抛光原理结构示意图

关于气囊抛光技术近些年的发展, 包括去除机 理的微观解释, 抛光垫磨损效应研究, 气囊抛光装 备发展趋势等。BO 等 ${ }^{[129]}$ 试验研究了不同磨损程度 的气囊抛光工具头抛光垫对材料去除的影响机理, 结合摩擦因数与力的变化进一步解释材料去除机 理。PAN 等 ${ }^{[130]}$ 从摩擦磨损的角度, 试验研究了气 囊抛光主要工艺参数变化对材料去除的影响规律, 并通过摩擦因数与力的变化进一步解释材料去除机 理。CAO 等 ${ }^{[131]}$ 和厦门大学 $\mathrm{SHI}$ 等 ${ }^{[132]}$ 也研究了气囊 抛光材料去除机理, 但均侧重于微观尺度分析, 此 外, 前者提出的微观模型基于 Preston 模型, 材料去 除与工艺参数仍是线性关系, 后者提出的模型进一 步解释了材料去除与工艺参数之间的非线性关系。 SHI 等 ${ }^{[133]}$ 结合抛光垫磨损效应, 从微观尺度解释了 气囊抛光过程材料去除规律, 图 30 为气囊抛光材料 去除的微观理论模型。 


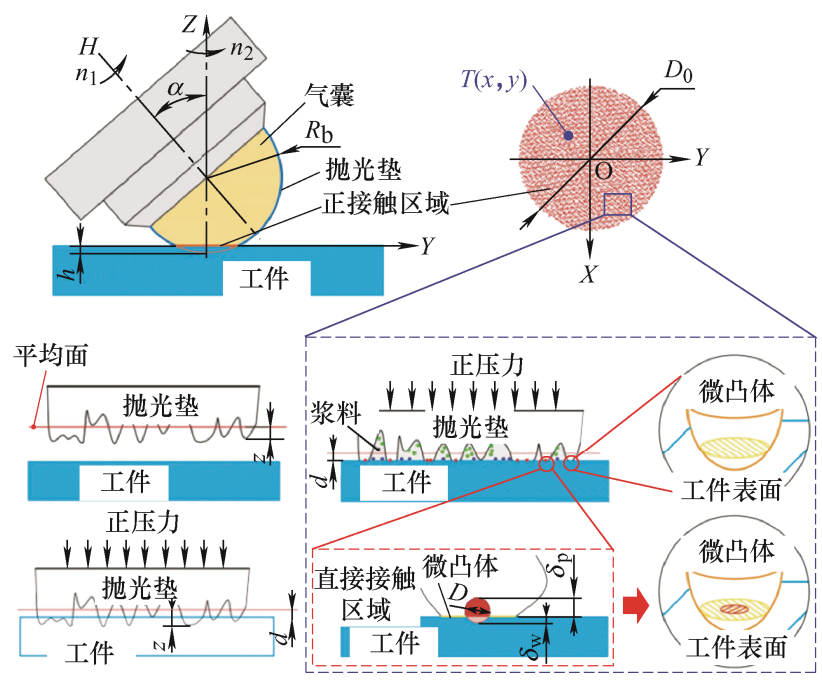

图 30 气囊抛光材料去除的微观理论模型

厦门大学智能制造与精密工程实验室团队在气 囊抛光专门装备与机器人气囊抛光系统研制方面都 开展了相关工作。图 31 为该团队研制的气囊抛光机 床 BP480, 已实际投入生产使用。近年来, 机器人 气囊抛光也是一个新的发展趋势。基于机器人自身 的特点, 将工业机器人与气囊技术结合应用于光学 材料的抛光, 既可以满足光学材料高效抛光中对工 具的效率和机器本体的低成本、高灵活性的需求, 又解决了气囊抛光技术推广对于机器本体低成本和 控制简单的要求, 是极具潜力的装备研制方案。 ZHONG 等 ${ }^{[134]}$ 建立了机器人气囊抛光进动控制模 型, 研究机器人气囊抛光的运动误差补偿, 以提升 大口径非球面光学组件的预抛光加工精度。试验验 证了理论模型的正确性与误差补偿方法的有效性。 为了避免多机器人气囊抛光系统中机器人之间的移 动空间相互重叠, QIN 等 ${ }^{[135]}$ 提出了碰撞检测算法。 通过球体边界框简化了多机器人气囊抛光系统模 型, 在简化模型中, 将碰撞检测问题转化为边界框 相交的判断问题。试验验证了算法的有效性。
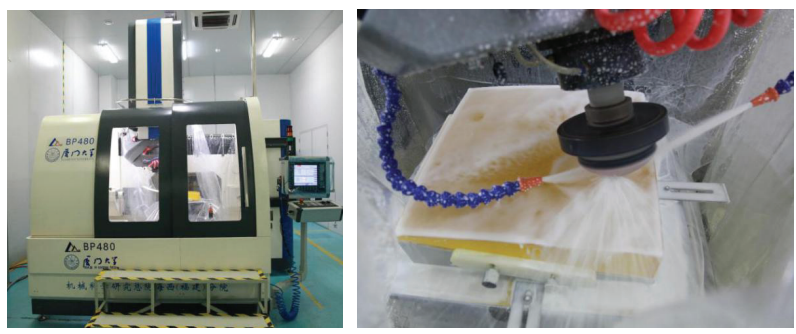

图 31 厦门大学研制气囊抛光机床 BP480

厦门大学智能制造与精密工程实验室团队在机 器人气囊抛光系统研制方面也取得了进展。主要研 究内容及创新工作如下。

(1) 搭建工业机器人气囊快速抛光系统, 实现
快速抛光平台开发。基于气囊进动抛光原理和工业 机器人编程控制要求, 建立机器人气囊进动运动数 学模型, 提出控制算法实现机器人气囊抛光系统的 进动抛光控制, 并验证其准确性。图 32 为搭建的机 器人气囊抛光系统。
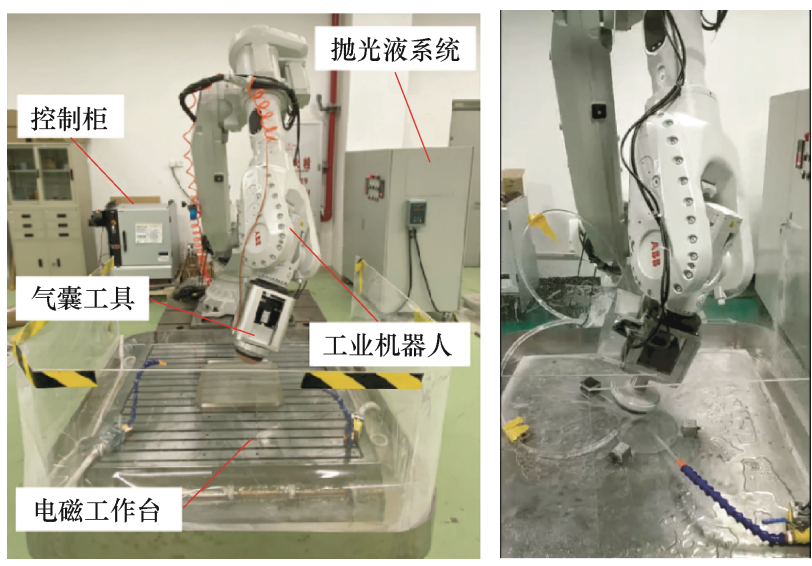

图 32 搭建的机器人气囊抛光系统

(2) 实现工业机器人末端笛卡儿刚度矩阵建 模, 分析气囊抛光过程机器人末端载荷情况, 仿真 获得不同姿态下气囊抛光机器人末端变形; 建立含 误差的气囊抛光材料去除函数, 分析定点抛光下多 步离散进动抛光的稳定性, 并采用试验验证系统抛 光稳定性。图 33 为考虑末端变形的实际去除函数仿 真结果, 图 34 为不同位置抛光区域 $X$ 方向轮廓线 对比。

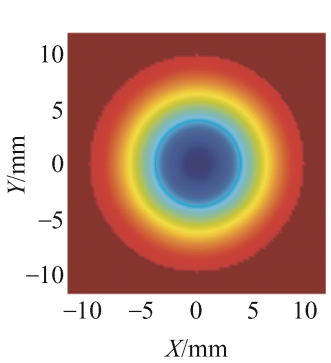

(a) 去除函数三维示意图

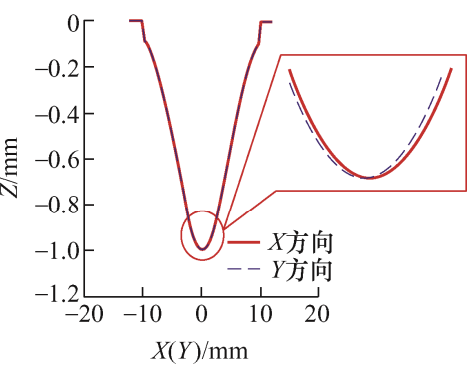

(b) 去除函数 $X Y$ 方向轮廓线
图 33 考虑末端变形的实际去除函数仿真结果

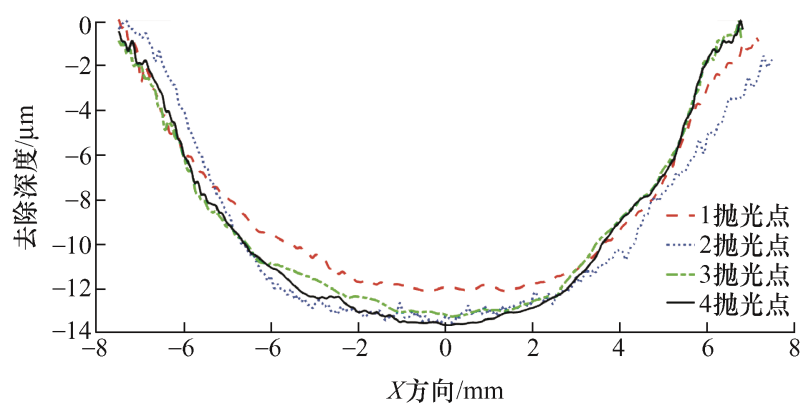

图 34 不同位置抛光区域 $X$ 方向轮廓线对比

(3) 设计机器人气囊抛光工艺试验, 完成光学 
组件的直线和平面区域的均匀抛光试验, 验证机 器人气囊抛光系统连续加工能力; 并仿真分析机 器人误差对连续加工的影响, 为后续研究提供理 论依据。图 35 为机器人气囊平面均匀抛光测量结 果, 从测量结果可见, 单步抛光和四步离散抛光 后对工件表面均具有比较好的抛光效果, 可迅速

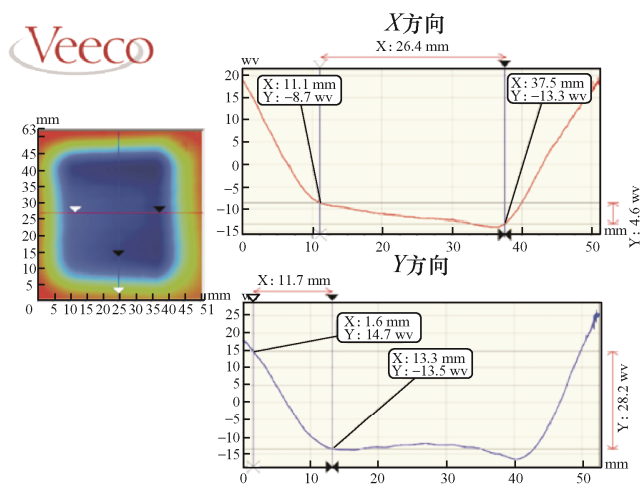

(a) 单步斜抛平面面形测量结果
降低表面粗糙度, 并且有较高去除效率。另外, 对比数控五轴气囊抛光机床与机器人气囊抛光系 统轨迹误差的影响, 分别对其理想抛光轨迹引入 $10 \mathrm{~mm}$ 和 $1 \mathrm{~mm}$ 的随机误差, 即驻留点可能位置 在以理论驻留点为圆心随机误差为半径的圆内, 整面去除效果如图 36 所示。

图 35 机器人气囊平面均匀拋光测量结果

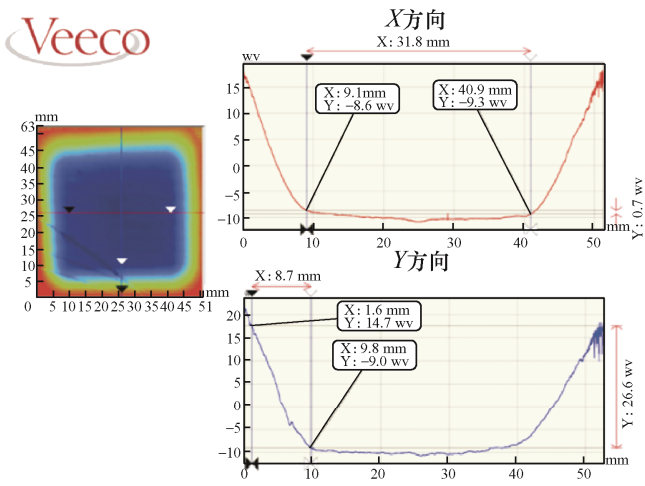

(b) 四步离散抛光平面面形测量结果

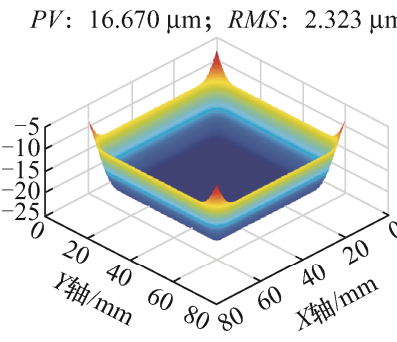

(a) 气囊均匀抛光理想仿真结果

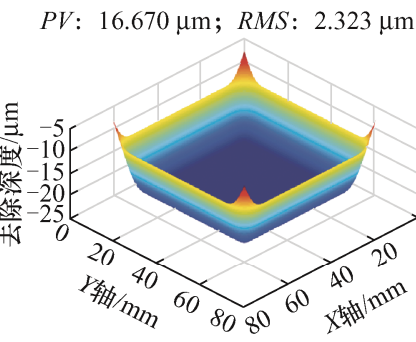

(b) 数控机床含误差仿真结果

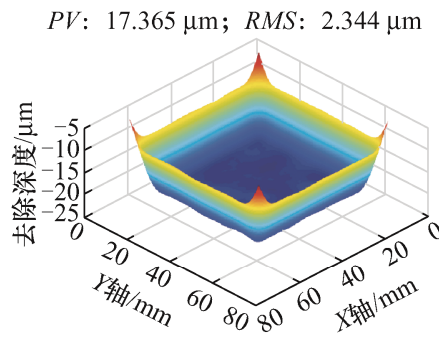

(c) 机器人气囊抛光系统含误差仿真结果

图 36 气囊均匀抛光仿真结果对比

\section{4 振动辅助抛光}

为了提高抛光效率, 在目前已知的光学抛光加 工手段中, 针对平面和球面工件, 采用的主要方法 是用大抛光盘对整个光学平面进行全局抛光; 而对 于非球面工件, 由于其工件表面各点曲率半径不一 致, 全局抛光方法无法应用, 常用的加工方式是利 用小抛光盘进行局部抛光。但是这种方法加工效率 较低, 同时会引入较多新的中高频误差, 无法满足 现代光学系统对光学零件的要求。利用振动辅助抛 光是提高抛光效率的解决方法之一。

振动辅助抛光是一种新型的抛光技术, 振 动辅助抛光技术是在传统抛光过程中增加了外 部能量, 在刀具或工件上产生高频、低振幅的 振动, 从而提高加工效率, 提高表面质量和形状 精度。振动辅助抛光技术利用压电驱动器或磁致 伸缩驱动器将振动传递至变幅杆放大振幅, 驱动 工具头或者工件, 在抛光压力的作用下, 抛光盘
运动时会带动大量磨粒做高频振动, 不断冲击加 工工件表面，达到去除工件表面材料的效果

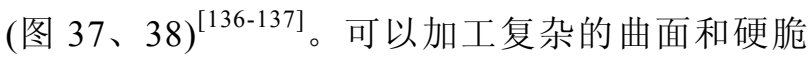
性材料等, 有效地改善工件的表面质量和精度。 目前的振动技术类型主要有: 轴向振动辅助抛 光; 径向振动辅助抛光; 两维振动辅助抛光; 三 维振动辅助抛光 ${ }^{[138]}$ 。

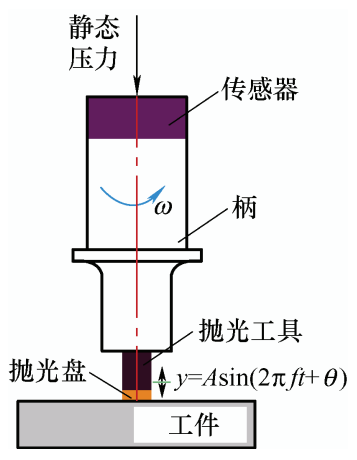

图 37 振动辅助抛光原理 


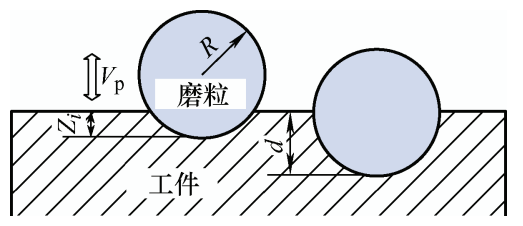

图 38 磨粒冲击工件表面数学模型

数学模型方面。LI 等 ${ }^{[139]}$ 建立了基于磨料对光 学玻璃玻璃表面压痕量的数学模型。模拟结果表明, 振动可以通过提高垂直方向振动幅值来提高材料去 除率, 而水平方向振动对提高材料去除率的作用较 小。李晓奥等 ${ }^{[140-141]}$ 完成了轴向振动辅助抛光加工 数学模型建立与分析, 从理论上分析了振动辅助抛 光的加工过程, 通过建立单颗磨粒冲击工件的数学 模型, 分析了振动参数变化引起硬脆材料的塑-脆去 除方式转变机制。

工艺优化方面。武昌壕 ${ }^{[142]}$ 进行了超硬微结构光 学表面振动辅助抛光工艺研究, 研究结果表明超声 振动辅助抛光在提高微结构抛光效率的同时还能够 有效地降低表面粗䊁度、提高面形精度。YU 等 ${ }^{[143]}$ 介绍了一种用于光学玻璃抛光的超声波振动辅助系 统, 研究了在加工过程中引入超声波振动将改变工 艺参数对加工结果的影响。

装备开发与抛光技术方面。目前振动系统主 要选用压电式和磁致伸缩式两种执行器。SUZUKI 等 ${ }^{[144]}$ 提出了两轴超声振动辅助抛光方法, 研制出了 一种带压电执行器的超声波双轴振动辅助抛光机。 对用无粘结碳化铇制作的微非球面模具进行了抛 光, 得到了 $8 \mathrm{~nm}$ 的表面粗䊁度和低于 $100 \mathrm{~nm} \mathrm{PV}$ 的成形精度。但是该方法受限于需要在谐振模式下 产生超声振动, 其实际工作频率受限于设计装置的 模态固有频率, 同时存在二维运动之间相互耦合的 问题, 解耦困难, 合成的二维运动轨迹难以精确控 制与预测。当振动幅值较大时, 会引起超声换能器 高频共振, 影响二维运动轨迹的控制与预测。谐振 模式具有高效利用能量的优点, 但只能在一定的频 率下工作。与谐振模式相比, 非谐振系统具有频率 可调、行程可控等优点。冯洁 ${ }^{[145]}$ 提出了非谐振式旋 转振动辅助磁流变抛光方法, 研制了一种旋转振动 辅助磁流变抛光装置。SONG 等 ${ }^{[146]}$ 为了解决两个方 向上振动耦合问题提出了解耦非共振型二维振动驱 动抛光工具作闭合往复重叠运动的原理, 研发了一 种基于两个相互垂直的超声振子的二维超声驱动抛 光系统。 $\mathrm{GU}$ 等 ${ }^{[147-148]}$ 开发了一种非共振振动辅助抛 光(VAP)来提高碳化硅 $(\mathrm{SiC})$ 陶瓷的表面粗糙度, 此
外其团队研制出一种由压电驱动器驱动的具有二次 杜杆放大机制的 2-DOF 振动辅助抛光装置, 用于 $X Y$ 平面上产生椭圆振动, 获得振动辅助抛光。CHEE 等 ${ }^{[149-150]}$ 提出并开发了一种压电驱动器驱动的二维 低频振动抛光驱动器。在该柔性装置的放大作用下, 刀具沿圆形轨迹振动, 达到抛光效果。在此基础上, CHEE 等 ${ }^{[150]}$ 开发了一种三维低频振动(3DLFV)抛光 驱动器。WANG 等 ${ }^{[151]}$ 提出了一种新型的三维振动 辅助抛光技术, 并进一步研究了加工结构表面的抛 光轨迹规划。该抛光系统由机床和三维振动装置两 部分组成。机床提供加工路径, 三维振动装置提供 振动轨迹。结合加工轨迹和振动轨迹, 巧妙地生成 三维抛光轨迹。

GUO 等 ${ }^{[152-153]}$ 利用磁致伸缩原理开发了一种新 型磁致伸缩振动辅助抛光机, 提出并发展了振动辅 助抛光(VAP)方法。在以往振动辅助磁性磨料抛光 (VAMAP)的研究中, 磁性复合悬浮液无法进入微特 征的角落, 无法产生均匀的压力分布, 因此在有效 降低表面粗粘度的同时保持微特征的形式存在一定 的局限性。为了解决这一问题, GUO 等 ${ }^{[154]}$ 提出了 一种振动辅助磁研磨抛光(VAMAP)方法, 该方法具 有抛光微特征表面的能力。然而, 它仅限于具有直 微特征的非磁性或微磁性材料。在此基础之上, GUO 等 ${ }^{[155]}$ 提出了一种新的局部化 VAMAP 方法。 它可以用于抛光弯曲微特征, 工件材料可以是铁磁材 料。该方法使用疏松磨料, 在保持微特征形态的同时, 实现了纳米级表面粗粘度和无损伤表面(图 39)。

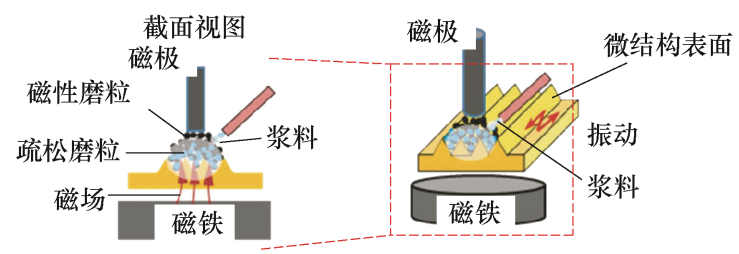

图 39 VAMAP 方法原理

\section{5 剪切增稠抛光}

剪切增稠抛光是近年来兴起并逐渐发展的一项 新型抛光技术, 于 2015 年由湖南大学李敏等率先提 出, 它是利用非牛顿流体抛光液的剪切增稠流变特 性达到增强把持磨粒的约束力, 形成类似柔性固着

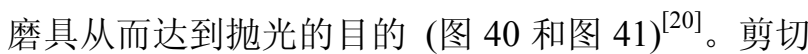
增稠抛光具有很强的表面平滑能力, 并且其抛光过 程是非接触式的, 不会与工件直接接触, 不会造成 表面损伤, 因此剪切增稠抛光在光学制造方面极具 潜力 ${ }^{[156-161]}$ 。 


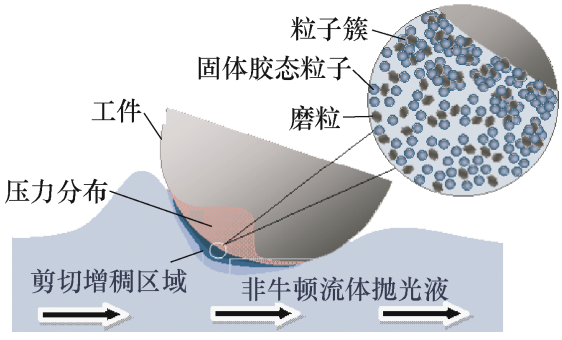

图 40 基于非牛顿流体剪切增稠效应的抛光方法

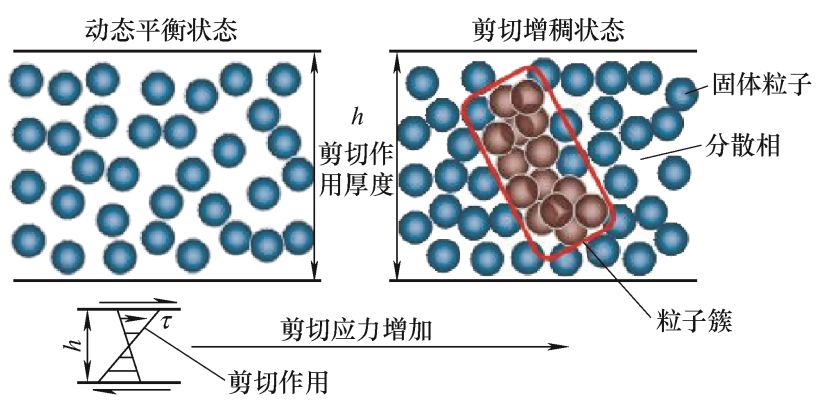

图 41 非牛顿流体剪切增稠效应原理示意图

剪切增稠作为一种确定性抛光方法, 其材料 去除也是可控的。针对剪切增稠的抛光方法, 李 敏 ${ }^{[20]}$ 基于粒子簇理论 ${ }^{[162]}$ 首先建立了剪切增稠抛光 技术的材料去除模型并进行了试验验证，如图 42 所示 ${ }^{[20]}$, 李敏将剪切增稠过程中的材料去除过程分 为 6 个阶段: (1) 无剪切增稠; (2) 剪切增稠; (3) 磨 粒剪切去除较小的切屑; (4) 剪切增稠效应增强去除 较大的切屑; (5) 材料去除完成; (6) 粒子簇分散等 过程。并结合 Preston 方程及流体动力学理论建立了 剪切增稠抛光的材料去除数学模型, 经验证其材料 去除模型与试验结果具有较好的一致性, 误差值仅 为 $6.12 \%$ 。

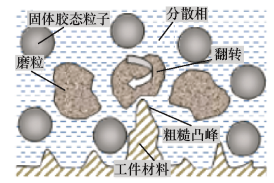

(a)

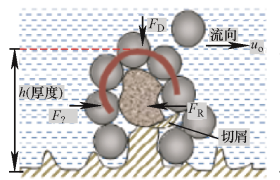

(d)

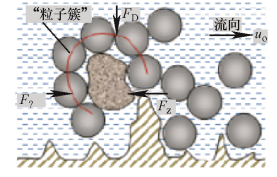

(b)

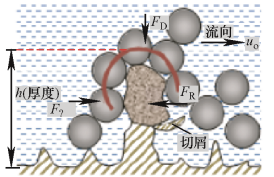

(e)

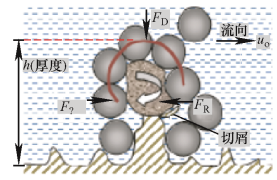

(c)

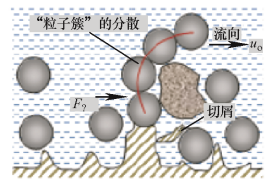

(f)
图 42 剪切增稠抛光微观图

$$
M R R=k_{0} \frac{3 n K}{R}\left(\frac{U}{h_{0}}\right)^{n+1} f(n, \varphi)
$$

式中, $U$ 可由式 $U=2 \pi \omega l$ 计算得出 $(\omega$ 选准主轴的 转速, $l$ 是主轴中心到工件表面的距离); $f(n, \varphi)$
是关于 $n$ 和 $\varphi$ 的函数; $R$ 为目标加工工件表面的 半径。

此外针对剪切增稠抛光过程中的表面演化过 程，李敏根据工件布氏硬度、剪切增稠机制以及塑 型压痕的磨料磨损理论建立了剪切增稠抛光过程中 的表面粗粘度预测模型。经试验验证其预测模型误 差仅为 $8.29 \%{ }^{[20]}$ 。

$$
R_{a}^{m}=R_{a}^{i}-\frac{3 u d_{s} \rho_{s} m_{a-s} p_{d}^{3} K_{p}^{2}}{4 \pi \rho_{a} H_{\mathrm{HBW}}^{3} l_{w} d_{a}^{5}}
$$

式中, $R_{a}^{m}$ 是加工对象最终粗粘度, $R_{a}^{i}$ 为加工对象初 始表面粗粘度, $u$ 为抛光速度可由工件放置位置求 得, $\rho_{s}$ 为抛光液的稀释浓度, $d_{s}$ 为抛光液的稀释率, $m_{a-s}$ 为抛光液中磨粒的浓度, $\rho_{a}$ 为磨粒密度, $p_{d}$ 动 压力, $l_{w}$ 为工件长度, $H_{\mathrm{HBW}}$ 为工件硬度, $k_{p}$ 和 $k_{\eta}$ 为剪切增稠抛光液特性相关的参数, $d$ 为磨粒平均 粒径。

$\mathrm{ZHU}$ 等 ${ }^{[161]}$ 提出了一种新的非牛顿流体子孔径 确定性抛光工艺(图 43), 能够以非接触方式实现超 光滑表面。并针对提出的抛光方法进行了建模和仿 真, 因为其抛光头用工件的间隙极小 $(0.06 \sim$ $0.21 \mathrm{~mm}$ ), 作者将工作区域的流体简化为薄层, 并 结合 Navier-Stokes 方程与第四强度理论建立了材料 去除模型。

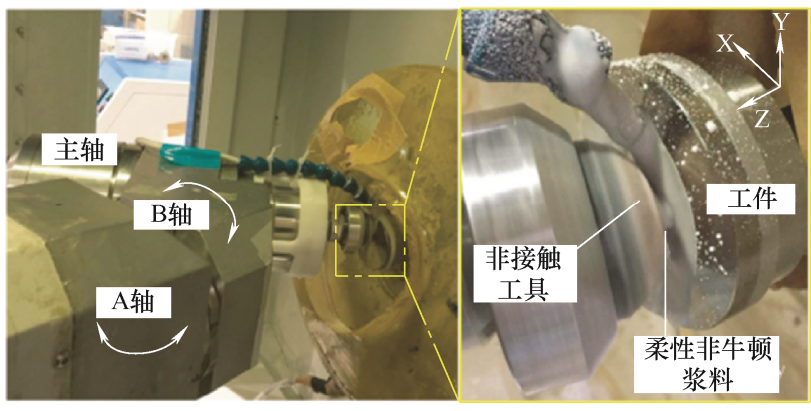

图 43 剪切增稠子孔径确定性抛光方法

$$
M R R=K \cdot \oiint P_{p}(x, y) \cdot V(x, y)^{m}
$$

式中, $K$ 为修正后的 Preston 系数, $m$ 定义了圆形区 域的速度幂指数 $V$ 。

与其他超精密抛光技术不同, 由于剪切增稠抛 光技术的特殊性，其抛光液往往也是特制的。一般 来说, 具有剪切增稠效应的抛光液一般包含磨粒、 剪切增稠相和去离子水等。李敏 ${ }^{[20]}$ 率先使用浓度 $23 \mathrm{wt} \%$, 平均粒径 $3.75 \mu \mathrm{m}$ 的氧化铝磨粒, 将多羟 基聚合物作为剪切增稠相配置了具有剪切增稠效应 的抛光液。并通过在 Cr12Mo1V1 模具钢圆柱工件 
进行试验, 其粗粘度从 $105.95 \mathrm{~nm}$ 降至 $5.1 \mathrm{~nm}$, 其 研究结果为剪切增稠抛光在光学领域的应用奠定了 基础。秦琳等 ${ }^{[163]}$ 使用 $9 \mathrm{wt} \% 12 \mathrm{~nm}$ 粒径的 $\mathrm{SiO}_{2}$ 为 溶质, 分子量 200 的聚乙二醇为溶剂制备了剪切增 稠基液, 并加入 $18 \mathrm{wt} \%$ 的 $\mathrm{CeO}_{2}$ 磨料对 $\mathrm{K} 9$ 玻璃进 行抛光, 获得了 $R a 1.023 \mathrm{~nm}$ 的良好效果(表 1)。WU 等 ${ }^{[164]}$ 通过采用将沸石咪唑酸酯(ZIF-8)纳米颗粒分 散到乙二醇(EG)中的方法研制了一种新型剪切增稠 液(Z-STF), 并通过改变 ZIF-8 纳米颗粒的形态使 Z-STF 的流变性能得到控制。ZHU 等 ${ }^{[161]}$ 使用淀粉 和聚合物悬浮液作为剪切增稠基液, 使用\# $6000 \mathrm{Al}_{2} \mathrm{O}_{3}$ 作为磨粒配置了一种新型剪切增稠抛光 液对纯镍进行抛光获得了 $R a 3.9 \mathrm{~nm}$ 的表面粗粘度。 其试验还表明, 与只有淀粉作为基液的抛光液相比, 采用淀粉和聚合物悬浮液的混合体系在获得具有光 学质量的无刮擦表面方面具有优势 ${ }^{[165-168]}$ 。针对软 脆光学材料 KDP, LI 等 ${ }^{[165]}$ 通过在抛光液中添加剪 切增稠相提出了一种新的无水基剪切增稠抛光 (ASTP) 方法, 如图 44、45 所示, 抛光 $20 \mathrm{~min}$ 后获 得了表面粗粘度为 $1.37 \mathrm{~nm}$ 的超光滑表面。抛光后 的 KDP 晶体表面精度达到 $0.68 \lambda(R M S)$ 。试验证明 ASTP 是一种潜在的 KDP 晶体超精密加工方法。LI 等 ${ }^{[169]}$ 通过采用在剪切增稠抛光液中加入具有两 亲性质的热敏粒子(n-异丙基丙烯酰胺和丙烯酸十 八酯的共聚物)的方法, 实现了抛光液的梯度增稠, 并使用 $0.6 \mu \mathrm{m} \mathrm{CeO}_{2}$ 磨粒最终获得优于 $1 \mathrm{~nm}$ 表面粗 䊁度和更小的表面/亚表面损伤(图 46)。

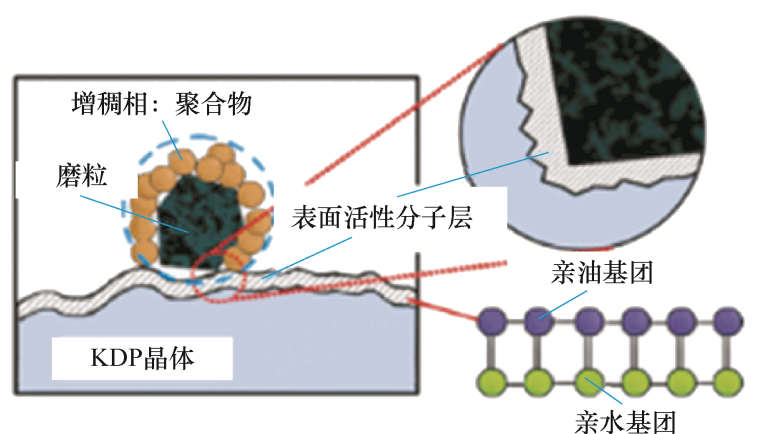

(a) 亲水亲油基团的影响

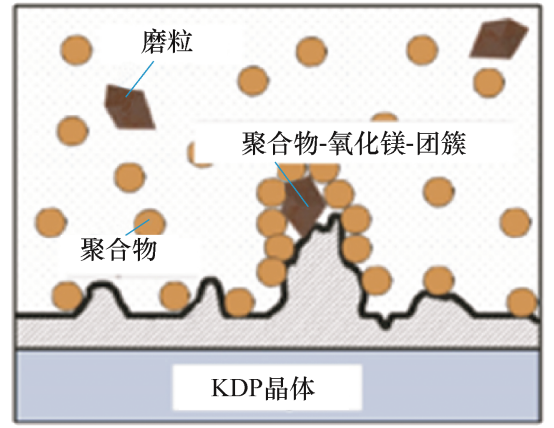

(b) 形成的聚合物-氧化镁-团簇

图 $44 \mathrm{KDP}$ 晶体无水剪切增稠抛光原理

表 1 不同体系剪切增稠抛光液达到的抛光效果

\begin{tabular}{cccc}
\hline 序号 & 分散介质及浓度 & 分散相 & 工件 \\
\hline 1 & $\mathrm{CeO}_{2} 18 \mathrm{wt} \%$ & $\mathrm{PEG}-200$ & $\mathrm{~K} 9$ 玻璃 \\
2 & $\mathrm{Al}_{2} \mathrm{O}_{3} 30 \mathrm{~g} / \mathrm{L}$ & 多羟基聚合物聚丙烯酰胺 & 镀镍平面件 \\
3 & $\mathrm{MgO} 25 \mathrm{wt} \%$ & $\mathrm{KDP}$ 晶体平面件 & 聚合物 \\
4 & $\mathrm{CeO}_{2} 12 \mathrm{wt} \%$ & $\mathrm{~N}$-异丙基丙烯酰胺和丙烯酸十八酯的共聚物 & 铌酸锂芯片 \\
\hline
\end{tabular}

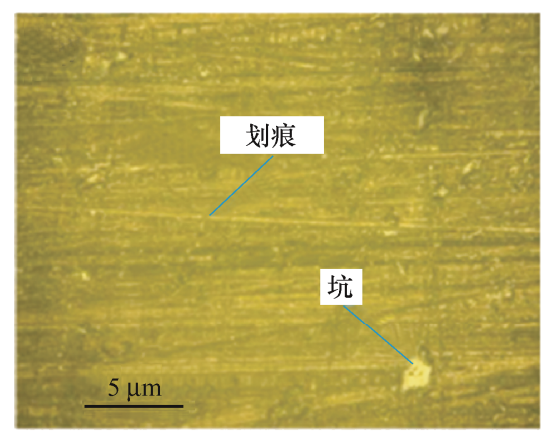

(a) 抛光前

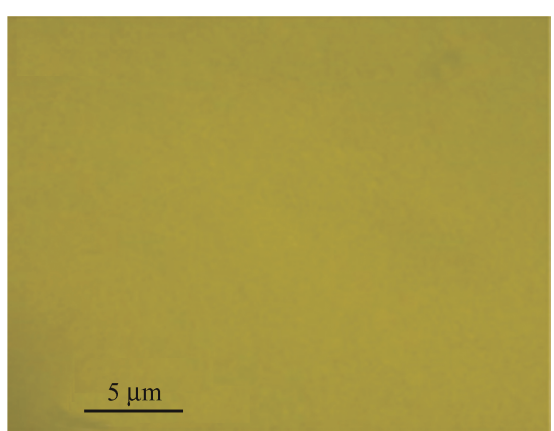

(b) 抛光后

图 45 无水剪切增稠抛光前后表面形貌 


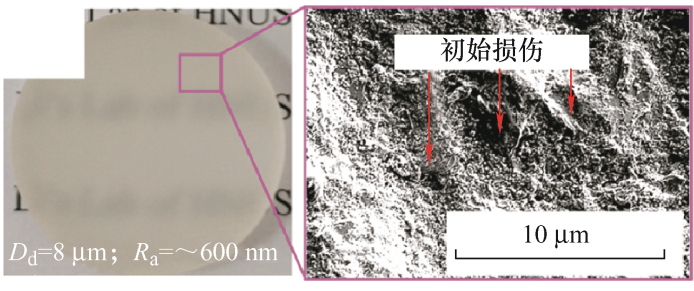

(a) 抛光前

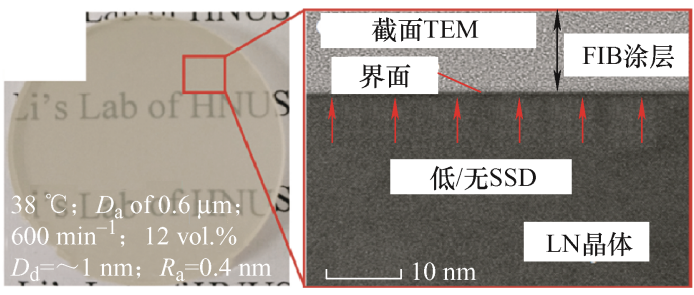

(b) 抛光后

图 46 AS-GTP 抛光前后的表面完整性

随着超精密加工技术的不断发展, 人们对光学 表面提出了更高的要求, 具体为抛光效率和表面质 量的要求。因此, 剪切增稠技术也不断发展出了一 些组合技术。如表 2 所示, 翁海舟 ${ }^{[170]}$ 采用剪切增稠 电解复合抛光方法抛光抛物面反射镜(304 不锈钢), 经剪切增稠电解复合抛光加工 $20 \mathrm{~min}$ 后, 工件表面 粗粘度由 $R a 270.654 \mathrm{~nm}$ 下降至 $R a 2.985 \mathrm{~nm}$ 。并发 现剪切增稠电解复合抛光的加工效率是剪切增稠抛 光的 4 5 倍。WEI 等 ${ }^{[171]}$ 通过使用在 AFM 油溶性 介质中添加剪切增稠相(瓜尔胶水凝胶基介质)的方 法, 利用剪切增稠效应大大提高了 AFM 的加工效 率, 由起初的 $69 \mathrm{mg} / \mathrm{h}$ 增加至 $351 \mathrm{mg} / \mathrm{h}$, 表面粗粘 度获得改善, 如图 47 所示。

表 2 剪切增稠复合技术达到的抛光效果

\begin{tabular}{|c|c|c|c|c|c|}
\hline 序号 & 复合技术 & 加工对象 & 初始表面粗糙度 & 最终表面粗楉度 & 效率 \\
\hline 1 & 电解抛光 & 304 不锈钢 & $R a 270.654 \mathrm{~nm}$ & $R a 2.985 \mathrm{~nm}$ & 是单一剪切增稠的 $4 \sim 5$ 倍 \\
\hline 2 & AFM & AISI316 不锈钢 & $R a 120.12 \mathrm{~nm}$ & $R a 6.48 \mathrm{~nm}$ & $\begin{array}{c}\text { 效率由 } 69 \mathrm{mg} / \mathrm{h} \text { 增加至 } 351 \\
\mathrm{mg} / \mathrm{h}\end{array}$ \\
\hline
\end{tabular}

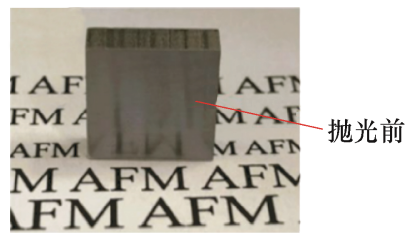

(a) 前

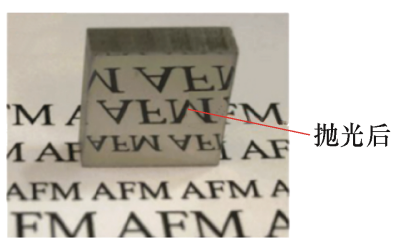

(b) 后
图 47 采用瓜尔胶水凝胶基介质在 $\mathrm{AFM}$ 前后的工件表面

剪切增稠抛光作为一种确定性抛光方法, 与传 统的接触式抛光方法相比, 由于其非接触和不会造 成表面损伤的优势, 是一种非常有希望的光学制造 技术。从剪切增稠抛光技术的提出到近年来的不断 发展优化, 其实现的表面粗粘度在不断地降低, 一 步步证实了其在光学制造领域应用的潜力。但是由 于剪切增稠抛光是一个极其复杂的过程, 涉及抛光 液的制备、材料去除机理的分析、材料去除率模型 的建立等, 因此对剪切增稠抛光方法的研究还有待 深入。通过分析近年来相关领域的研究发现剪切增 稠抛光方法要实现在光学制造领域更广泛的应用需 要在抛光液的制备方面做出改善, 如添加具有表面 平滑作用的增稠相、非单一增稠作用聚合物等实现 对增稠作用的有效可控、改善现有抛光液增稠体系 的分散状态等都是未来研究的重点。

\section{6 流体动压抛光}

流体动压加工即弹性发射加工, 简称为 EEM。
是日本的 MORI 等 ${ }^{[172]}$ 最早提出的超精密加工方法, 它将聚氨基甲酸乙酷回转球与工件一起置于悬浮 液含微细粉末粒子中, 利用回转球与工件表面之 问产生的流体润滑现象，达到去除工件表面材料 的目的。

而在最近十年的发展过程中, 基于弹性发射加 工原理的加工机理研究较多(图 48) ${ }^{[173]}$, 本节把加工 机理分为三个部分, 第一部分为去除机理的研究, HASHIMOTO 等 ${ }^{[174]}$ 提及了磨粒对工件表面之间的 作用方式有: 疲劳、剪切和滚压三种, 而具体哪种 方式发生作用取决于磨粒撞击工件的角度(磨粒速 度和工件法线的夹角)和磨粒自转速度。李岩 ${ }^{[175]}$ 发 现中高频误差难以去除的原因是额外去除量较小的 情况下，去除函数对空间波长小于其带宽的误差不 具备通过去除函数累加进行去除的能力。流场特性 的研究包括胡绍波 ${ }^{[176]}$ 研究了喷嘴大小, 流场分布, 喷射角度等对于效率和表面质量的影响。刘笑 ${ }^{[177]}$ 在抛光过程中将磨料流往复式加工改为内循环式, 并且研制新型水基黏弹性化学机械抛光流体。他们 分别对流场的流速、流动角度和流体的流动方式进 行研究进而实现抛光的目标。而随着近些年 CFD 技 术的不断更新和完善, 流场仿真成为分析流场的关 键过程, 刘文龙 ${ }^{[178]}$ 针对盘式动压抛光提出了通用型 模型, 并通过仿真证明 Tikhonov 正则化方法和类 迭代法均能提高面形精度, 为后续的实际加工提供 
了理论研究依据。刀彦飞 ${ }^{[179]}$ 在流体仿真结果中对求 得结果进行分析可以得知, 当采用压强入口时数值 计算结果与理论推导的结果是完全相符合的, 当采 用流量入口时数值计算结果略小于理论推导结果, 薛凯元 ${ }^{[180]}$ 基于 Fluent 平台对线性液动压抛光加工 中动压力的产生情况进行数值模拟, 并根据仿真结 果进行了辊子设计和工艺参数优化, 研究发现低转 速、大间隙下可以获得更均匀的动压力, 和更好的 表面质量, 但动压力较小, 材料去除率较低 (图 49)。而在仿真过程中不仅仅研究速度和压力场的变 化, 还有对于研究流场内碰撞模型及磨粒研究, 朱 胜伟 ${ }^{[181]}$ 采用固液两相流模型数值模拟了液动压流

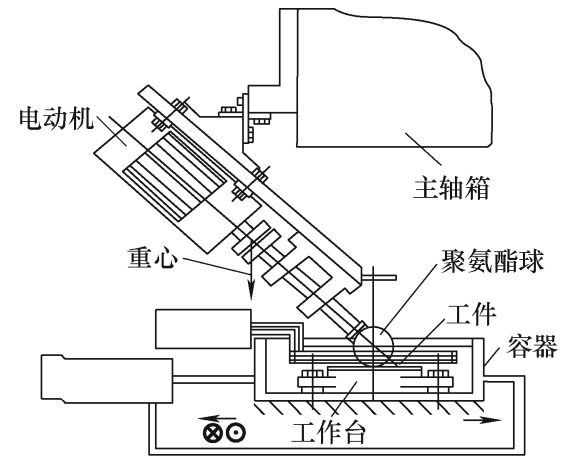

(a) 弹性发射抛光装置结构图
场和磨粒运动过程, 探索了加工试验参数对流体运 动产生的动压力和磨粒运动速度的影响规律。徐兴 芹, 2013 研究的是流场中流体运动和碰撞, 通过软 件 Fluent 对抛光区的微观流场进行了计算, 建立了 磨粒在流场中的运动方程, 建立了抛光液中磨粒 磨粒、磨粒工具轮以及磨粒工件的碰撞模型。WEN 等 ${ }^{[182]}$ 在此前研究的基础之上, 之后建立了基于计 算流体动力学 (CFD)的模型, 数值计算了抛光板旋 转引起的颗粒在目标表面附近的碰撞速度和碰撞 角。他们通过不同的方式去计算流体中磨粒的参数 和研究碰撞模型, 其研究思路和试验方法值得学习 和借鉴。

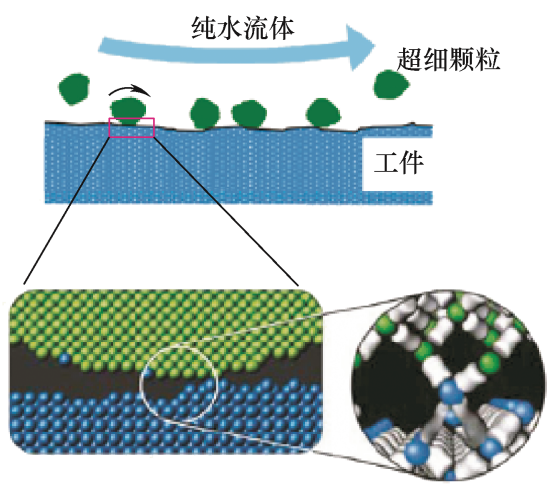

(b) 弹性发射材料去除机理

图 48 弹性发射加工装置及材料去除机理示意图

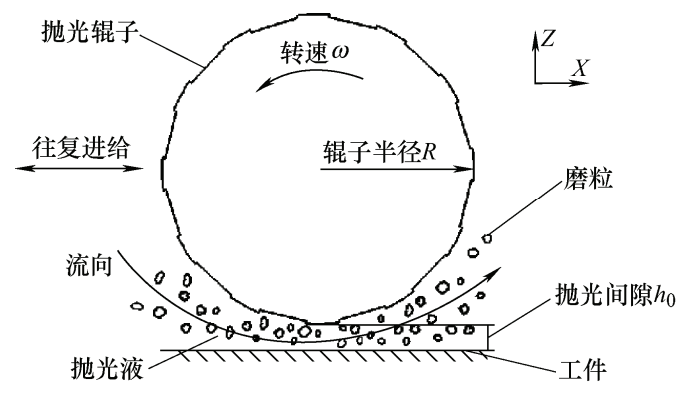

图 49 线性液动压抛光加工原理图

基于近十年里弹性发射加工原理的加工方式发 生了较大的变化, TAKEI 等 ${ }^{[183]}$ 年发表的文章中提 出方案并试验测试了通过在 EEM 中喷嘴出口和工 件表面之间引入聚焦流状态来控制静止点轮廓的形 状。李庆宇 ${ }^{[184]}$ 在参考彭文强的五轴联动控制上提出 了双转弹性发射加工技术, 让抛光工具在自转的同 时可以公转, 避免了工件旋转方式在加工位置上的 局限性, 在单转 EEM 的基础上, 能够使粗糙度值 进一步降低。二者均在现有的方案进行改进, 并通 过试验验证了改进方案的有效性。而加工方式发生 的变化近几年出现较多, 彭文强 ${ }^{[173]}$ 依靠五轴联动机 床进行位置及姿态的调整, 有助于精确地控制抛光
间隙，相对应早期的十字弹簧和之后广泛使用的压 电陶瓷和杜杆机构来调节间隙，无疑增加了抛光间 隙精确度。WEN 等 ${ }^{[182]}$ 设计了抛光盘的结构以形成 水动力应力的最优分布。然后制作了一种新型的抛 光装置。李岩 ${ }^{[175]}$ 年发表的文章中对光学组件新加工 方法进行了探索, 提出了基于中心供液流体动压原 理的盘式抛光技术，包括接触式中心供液盘式流体 动压抛光(CCDHDP)和浮式中心供液盘式流体动压 抛光(FCDHDP)。薛凯 ${ }^{[180]}$ 在文献[185]之前的装置 上进一步改进提出了采用圆柱形辊子作为抛光工 具, 搭建了线性液动压抛光加工试验平台, 对 $\mathrm{K} 9$ 玻璃进行了抛光加工试验, 试验结果表明线性液动 压加工具有超光滑表面加工能力, 加工后工件的表 面粗粘度良好。

对于不同工件材料和结构的可加工性探索, HIRATA 等 ${ }^{[186]}$ 研究发现在空间波长范围内, 玻璃柱 的表面粗粘度和误差从小于 $10 \mu \mathrm{m}$ 减小到 $100 \mu \mathrm{m}$, 并且证明了旋转球面 EEM 不仅适用于平面, 而且 适用于具有高平滑性能的柱面。彭文强 ${ }^{[173]}$ 在其文章 中通过试验提出了一种基于 EEM 下的水动力抛光 (HEP)试验, 并且研究了单晶硅和石英、非晶硅酸盐 
玻璃等不同类型光学材料的可加工性。

而对于表面粗糙度这一重要的表面质量指标, KANAOKA 等 ${ }^{[187]}$ 在其文章提出, 在抛光过程中让 工件旋转, 可以抑制误差, 使得工件旋转中心的粗 糙度达到 $0.05 \mathrm{~nm} R M S$ 。彭文强 ${ }^{[173]}$ 在研究中对石英 玻璃进行试验采用水动力抛光方法得到了 $R M S$ 粗 糙度为 $0.145 \mathrm{~nm}$ 的超平滑表面。李庆宇 ${ }^{[184]}$ 利用双 转弹性发射加工, 在单晶硅上将表面粗糙度值从 $0.636 \mathrm{~nm} R M S$ 降低到 $0.151 \mathrm{~nm} R M S$ 。郑子军等 ${ }^{[185]}$ 利用圆柱形辊子作为工具, 研究其材料去除机理, 把 $\mathrm{K} 9$ 玻璃表面粗楉度 $R a$ 从 $45.41 \mathrm{~nm}$ 下降到 $0.91 \mathrm{~nm}$ 。KAKAOKA 等 ${ }^{[187]}$ 合作开发了用于加工球 面和非球面的新一代 EEM 装置, 利用该装置已经 成功完成了对 EUVL 系统的非球面光刻物镜的超光 滑表面加工, 并且其表面粗糙度优于 $0.1 \mathrm{~nm} R M S$ 。

最后对于流体动压与其他技术相结合的部分, 本文也进行了整理。胡绍波 ${ }^{[176]}$ 把弹性发射加工和射 流加工进行了结合, 在加工过程中, 射流中的磨粒 在本身就获得了的射流压力和流体动压力场的共作 用下获得了足够的能量, 然后对表面进行冲击。此 方法可以弥补射流在发射之后产生的扩散作用, 提 高加工效率, 避免破坏非抛光表面的质量。PENG 等 ${ }^{[188]} 2018$ 年提出了把磁流变抛光 $(\mathrm{MRF})$ 和水动力 抛光(HEP)技术相结合的新工艺。MRF 具有较高的 确定性计算能力, 可以快速降低表面图形误差, 但 引入 MRF 抛光痕会使表面质量变差。材料对 HEP 的去除依赖于纳米粒子与工件在弹性模态下的化学 作用, 在保持良好的表面形貌的前提下, 可以很容 易地获得亚纳米级的超光滑表面。这两种方案把流 体动压加工和其他加工方案进行结合, 第一种方案 可以有效改善流体动压加工效率不高的缺点。第二 种则可以在保证加工形貌的情况下去得到超光滑表 面。流体动压抛光方式一般会在抛光的最后一个环 节使用, 因为其能达到原子级别的去除, 并且其加 工精度也是现阶段最高的。

上述各项光学超精密抛光方法由于其加工原 理、采用介质及可控参数形式上的差异导致不同方 法的技术难度、加工材料适应性、工具磨损、去除 函数稳定性、加工效率、亚表面损伤、边缘效应、 面形收敛性及其经济性方面都存在差异。大口径适 应性方面, 等离子体抛光、气囊抛光及剪切增稠抛 光都具有良好的效果, 而离子束抛光对大口径光学 组件的适应性一般。针对光学组件面形的适应性, 离子束和气囊抛光由于其去除函数精细和自适应的 优点针对非球面光学组件具有较好的面形适应效
果, 振动辅助抛光由于抛光介质的自适应特点在抛 光微结构光学组件时也具有较好的面形保持效果, 而由于剪切增稠抛光实现方式方面的限制目前对非 球面面形的适应性还较差。材料去除率方面, 气囊 抛光一般具有较高的抛光效率, 而振动辅助抛光、 剪切增稠抛光以及流体动压抛光效率一般, 离子束 抛光和等离子体抛光方法的去除效率极低。采用离 子束或等离子体抛光得到的光学组件或模具一般不 会造成亚表面损伤，而采用气囊抛光、剪切增稠抛 光和流体动压抛光得到的光学组件及模具都存在不 同程度的亚表面损伤。针对不同抛光方法的实现成 本, 气囊抛光、剪切增稠抛光、振动辅助抛光以及 流体动压抛光的实现成本都较低, 而离子束和等离 子体抛光由于其苛刻的运行条件及复杂的控制系统 实现成本较昂贵 ${ }^{[189-191]}$ 。

\section{4 总结与展望}

光学组件的超精密加工正向着高精度、高效率、 低成本的方向发展, 越来越多的光学组件已广泛应 用于光学成像、大功率激光系统、激光核聚变、空 间观测等领域。本文主要论述了先进光学超精密加 工技术近些年的研究进展, 系统介绍了超精密车削、 磨削和抛光具体技术的基本原理及其典型应用，并 对未来光学组件超精密加工技术进行展望。

(1) 本文首先综述了光学超精密切削技术的发 展情况。其中, 以单点金刚石车削技术最具代表性, 而辅助单点金刚石车削技术是近年来较为流行的技 术。在辅助技术的未来发展中, 应当进一步探究多 种辅助技术的组合应用对单点金刚石车削技术的影 响, 深入研究辅助技术对单点金刚石车削光学组件 的加工机理, 优化相应的工艺参数, 以期实现高质 量光学加工表面, 拓展超精密切削技术在光学领域 的应用范围。此外, 辅助单点金刚石车削技术的发 展仍然存在诸多挑战, 包括系统集成、复杂光学表 面加工的系统控制、在位或在线检测技术等，相应 研制开发可靠性高、稳定性强、智能数字化的机床 装备亦值得关注。

(2) 超精密磨削技术方面, 由于磨削加工后, 一般会在光学工件表面留下规则的磨削痕迹及亚表 面损伤, 因此, 仅采用超精密磨削加工难以满足非 球面光学组件表面质量越来越高的要求。所以，采 用磨抛技术相复合的方式进行光学组件的超精密加 工是未来先进光学制造的趋势之一。

(3) 超精密光学抛光技术方面, 传统的加工方 
法如等离子抛光、磨粒流抛光等, 通过装置改进或 与其他方法有效组合, 可以达到更高的材料去除率 并获得更高的表面质量。在超精密加工技术的未来 发展中, 复合加工技术可能是实现光学组件高精高 效加工的有效手段。近年来出现的新的抛光方法如 剪切增稠抛光和流体动压抛光具有非接触加工特 征, 能够获得较高的加工表面质量, 并对光学组件 亚表面损伤程度小, 在获得高表面完整性方面具有 明显优势。然而, 目前相关研究大多停留在理论层 面, 在实际应用研究方面仍具有很大潜力。在装备 研制方面, 以机器人气囊抛光系统为例, 需要开展 的工作包括刚度优化、颤振识别和优化、边缘去除 函数研究等。气囊抛光存在明显的边缘效应, 可基 于机器人气囊抛光系统建立有效的边缘去除函数, 进而提出边缘效应抑制控制算法, 提高机器人气囊 抛光的可靠性。对于具有特殊应用的光学组件如微 细结构光学组件, 因其表面质量和面形是主要加工 指标, 而目前的抛光方法大多存在一定不足, 或不 能达到理想的表面质量, 或不能获得良好的面形, 因此, 有必要研制新的抛光方法以获得符合要求的 微细结构光学组件。随着计算机数控技术的飞速发 展, 基于子口径的抛光方法在实现更高的面形精度 方面具有较大的优势。通过子口径抛光方法与先进 数控系统的有效结合, 有利于推动先进光学加工技 术的发展。

\section{参 考 文 献}

[1] GAO H, WANG X, GUO D, et al. Research progress on ultra-precision machining technologies for soft-brittle crystal materials $[\mathrm{J}]$. Frontiers of Mechanical Engineering, 2017, 12(1): 77-88.

[2] XIA Z, FANG F, AHEARNE E, et al. Advances in polishing of optical freeform surfaces: A review[J]. Journal of Materials Processing Technology, 2020 : 116828.

[3] 伍凡, 戴一帆. 先进光学制造专题导读 $[\mathrm{J}]$. 光电工程, 2020, 47(8): 2-3.

WU Fan, DAI Yifan. Introduction to advanced optical manufacturing[J]. Opto-Electronic Engineering, 2020, $47(8): 2-3$.

[4] 淡晶晶, 王传珂, 贺少勃, 等. 大科学工程与先进制造 业的双向驱动效应研究——以高功率固体激光装置研 制为例 $[J]$. 工程研究-跨学科视野中的工程, 2018, 10(5): 479-487.

DAN Jingjing, WANG Chuanke, HE Shaobo, et al.
Research on bidirectional-driven effects of large science project and advanced manufacture: Taking high power solid laser device as an example[J]. Journal of Engineering Studies, 2018, 10 (5): 479-487.

[5] 王建刚. 光学零件加工的现状及发展 [J]. 现代国企研 究, 2016(4): 205-207.

WANG Jiangang. Present situation and development of optical parts processing[J]. Modern SOE Research, 2016 (4): 205-207.

[6] 金滩, 李平, 肖航, 等. 大中型光学组件高效精密磨削 技术研究综述 $[J]$. 机械工程学报, 2016, 52(9): 165-175. JIN Tan, LI Ping, XIAO Hang, et al. Research summary on precision grinding technology oriented to achieve high process efficiency for large and middle-scale optic[J]. Journal of Mechanical Engineering, 2016, 52(9): 165-175.

[7] 于建海, 于秋跃, 房安利, 等. 大口径碳化硅反射镜高 效磨削实时补偿技术 $[\mathrm{J}]$. 光学技术, $2020,46(4)$ : 502-506.

YU Jianhai, YU Qiuyue, FANG Anli, et al. Real-time compensation of high efficiency grinding for the large aperture mirror[J]. Optical Technique, 2020, 46(4): 502-506.

[8] 朱日宏, 孙越, 沈华. 光学自由曲面面形检测方法进展 与展望[J]. 光学学报, 2021, 41(1): 0112001.

ZHU Rihong, SUN Yue, SHEN Hua. Progress and prospect of optical freeform surface measurement[J]. Acta Optica Sinica, 2021，41(1): 0112001.

[9] 郑爽. 高精度小口径非球面加工工艺研究[D]. 长春: 长 春理工大学, 2013.

ZHENG Shuang. The processing technology research of high precision minor-caliber aspheric surface[D]. Changchun: Changchun University of Science and Technology, 2013.

[10] 刘冬梅, 郑爽, 付秀华, 等. 高精度小口径非球面加工 工艺研究 $[J]$. 长春理工大学学报, 2013, 36(Z1): 24-27. LIU Dongmei, ZHENG Shuang, FU Xiuhua, et al. Research on the processing technology of high precision minor-caliber aspheric surface[J]. Journal of Changchun University of Science and Technology, 2013, 36 (Z1): 24-27.

[11] 徐志强. 小口径非球面斜轴磁流变抛光关键技术研究 [D]. 长沙: 湖南大学, 2015.

XU Zhiqiang. Study on key technology of inclined magnetorheological polishing for micro aspherical surface[D]. Changsha: Hunan University, 2015. 
[12] 杨辉, 李静, 张涁, 等. 某光学自由曲面棱镜超精密加 工技术研究[J]. 航空精密制造技术，2019，55(5)：1-6. YANG Hui, LI Jing, ZHANG Bin, et al. Study on ultra-precision machining technology of optical free-form prism[J]. Aviation Precision Manufacturing Technology, 2019, 55(5): 1-6.

[13] 姚庠. 光学微结构表面精密加工的关键技术研究 [D]. 上海: 华东理工大学, 2016.

YAO Xiang. Research for key surface processing method of optical microstructure[D]. Shanghai : East China University of Science and Technology, 2016.

[14] CHRIS J E, JAMES B B. “Structured” , “Textured” or "Engineered" surfaces[J]. CIRP Annals-Manufacturing Technology, 1999, 48(2): 541-556.

[15] 吴春亚, 郭闯强, 裴旭东, 等. 太赫兹段慢波结构的微 细加工技术研究新进展 [J]. 机械工程学报, 2019 , 55(7): 187-198.

WU Chunya, GUO Chuangqiang, PEI Xudong, et al. New progress of microfabrication techniques for slow wave structures at $\mathrm{THz}$ frequencies[J]. Journal of Mechanical Engineering, 2019, 55(7): 187-198.

[16] 孟晓辉, 王永刚, 李文卿, 等. 应用旋转法实现大口径 非球面反射镜零重力面形加工 $[\mathrm{J}]$. 光学精密工程, 2019, 27(12): 2517-2524.

MENG Xiaohui, WANG Yonggang, LI Wenqing, et al. Fabrication of zero-gravity surface for large-aperture aspherical mirror by using rotationally method[J]. Optics and Precision Engineering, 2019，27(12): 2517-2524.

[17] 徐放. 大口径轻量化铝反射镜加工工艺研究[D]. 南京: 南京理工大学, 2017.

XU Fang. Research on lightweight large aperture reflector processing technology[D]. Nanjing: Nanjing University of Science and Technology, 2017.

[18] BRINKSMEIER E, RIEMER O, GESSENHARTER A, et al. Polishing of structured molds[J]. Cirp Annals, 2004, 53(1): $247-250$.

[19] CHEUNG C F, KONG L B, HO L T, et al. Modeling and simulation of structure surface generation using computer controlled ultra-precision polishing $[\mathrm{J}]$. Precision Engineering, 2011, 35(4): 574-590.

[20] 李敏. 剪切增稠抛光方法的基础研究[D]. 长沙: 湖南大 学, 2015.

LI Min. Fundamental research on shear-thickening polishing method[D]. Changsha: Hunan University, 2015.

[21] IKAWA N, DONALDSON R, KOMANDURI R, et al.
Ultraprecision metal cutting: The past, the present and the future[J]. CIRP Annals, 1991，40(2): 587-594.

[22] 戴一帆, 周林, 解旭辉, 等. 应用离子束进行光学镜面 确定性修形的实现[J]. 光学学报, 2008(6): 1131-1135. DAI Yifan, ZHOU Lin, XIE Xuhui, et al. Deterministic figuring in optical machining by ion beam[J]. Acta Optica Sinica, 2008(6): 1131-1135.

[23] 唐瓦. 离子束抛光大口径非球面去除模型与工艺研究 [D]. 长春: 中国科学院长春光学精密机械与物理研究 所, 2016.

TANG Wa. Research on removal model and technology for ion beam figuring large aspherical mirror[D]. Changchun : Changchun Institute of Optics, Fine Mechanics and Physics of CAS, 2016.

[24] XU H, ZHANG X, XU M, et al. Study on the control of surface roughness in single point diamond turning[C]//6th International Symposium on Advanced Optical Manufacturing and Testing Technologies: Advanced Optical Manufacturing Technologies, 2012: 84161D.

[25] 郭海涛. KDP 晶体单点金刚石切削表面质量分析与工 艺参数优化研究[D]. 天津: 天津大学, 2018.

GUO Haitao. Study on the surface quality analysis and process parameter optimization of KDP crystal single point diamond cutting[D]. Tianjin: Tianjin University, 2018.

[26] ZHANG L, ZHOU W, NAPLES N J, et al. Fabrication of an infrared Shack-Hartmann sensor by combining high-speed single-point diamond milling and precision compression molding processes[J]. Applied Optics, 2018, 57(13): 3598-3605.

[27] WEI Y, ZHAI P, CHEN X, et al. Study on design and diamond turning of optical freeform surface for progressive addition lenses $[\mathrm{J}]$. Mathematical Problems in Engineering, 2020, 2020: 1-9.

[28] LEI X Y, ZHANG S, WANG S F, et al. Influence of the arrangement of vacuum chuck holes on the transmittance wavefront of large-aperture KDP in single-point diamond turning[J]. Applied Optics， 2020， 59(12): 3619-3623.

[29] HATEFI S, ABOU-EL-HOSSEIN K. Feasibility study on design and development of a hybrid controller for ultra-precision single-point diamond turning[J]. Majlesi Journal of Electrical Engineering, 2019, 13(2): 121-128.

[30] ZHANG L, ZHAO H, YANG Y, et al. Evaluation of repeated single-point diamond turning on the deformation 
behavior of monocrystalline silicon via molecular dynamic simulations[J]. Applied Physics A, 2014, 116(1): 141-150.

[31] HE C, ZONG W, SUN T. Origins for the size effect of surface roughness in diamond turning[J]. International Journal of Machine Tools and Manufacture, 2016, 106: $22-42$.

[32] HE C, ZONG W. Influencing factors and theoretical models for the surface topography in diamond turning process: A review[J]. Micromachines, 2019, 10(5): 288.

[33] ZHANG S, ZHANG H, ZONG W. Modeling and simulation on the effect of tool rake angle in diamond turning of KDP crystal[J]. Journal of Materials Processing Technology, 2019, 273: 116259.

[34] HATEFI S , ABOU-EL-HOSSEIN K. Review of non-conventional technologies for assisting ultra-precision single-point diamond turning[J]. The International Journal of Advanced Manufacturing Technology, 2020, 111(9-10): 1-19.

[35] MOHAMMADI H, RAVINDRA D, KODE S K, et al. Experimental work on micro laser-assisted diamond turning of silicon (111)[J]. Journal of Manufacturing Processes, 2015, 19(8): 125-128.

[36] MOHAMMADI H, POYRAZ H B, RAVINDRA D, et al. Single point diamond turning of silicon by using micro-laser assisted machining technique[C]/Msec., 2014.

[37] SHAHINIAN H, NAVARE J A, BODLAPATI C, et al. Micro laser assisted single point diamond turning of brittle and hard materials $[\mathrm{C} / \mathrm{CD}] / /$ Laser Applications in Microelectronic and Optoelectronic Manufacturing (LAMOM) XXV, 2020.

[38] NAVARE J, KANG D, ZAYTSEV D, et al. Experimental investigation on the effect of crystal orientation of diamond tooling on micro laser assisted diamond turning of zinc sulfide[J]. Procedia Manufacturing, 2020, 48: 606-610.

[39] ZHOU T, XU R, RUAN B, et al. Study on new method and mechanism of microcutting-etching of microlens array on $6 \mathrm{H}-\mathrm{SiC}$ mold by combining single point diamond turning with ion beam etching[J]. Journal of Materials Processing Technology, 2019, 278: 116510.

[40] LEE Y J, CHAUDHARI A, ZHANG J, et al. Thermally assisted microcutting of calcium fluoride single crystals[M]//ZHANG Junjie, GUO Bing, ZHANG Jianguo. Simulation and experiments of material-oriented ultra-precision machining. Berlin: Springer, 2019: 77-102.

[41] ZHANG S, ZHOU Y, ZHANG H, et al. Advances in ultra-precision machining of micro-structured functional surfaces and their typical applications[J]. International Journal of Machine Tools and Manufacture, 2019, 142: 16-41.

[42] 李荣涁, 孔令豹, 张志辉, 等. 微结构自由曲面的超精 密单点金刚石切削技术概述 [J]. 机械工程学报, 2013, 49(19): 144-155.

LEE Wingbun, KONG Lingbao, CHEUNG Chifai, et al. An overview of ultra-precision diamond machining of microstructured freeform surfaces[J]. Journal of Mechanical Engineering, 2013, 49 (19): 144-155.

[43] ZHANG Guoqing, TO S, XIAO Gaobo. A novel spindle inclination error identification and compensation method in ultra-precision raster milling[J]. International Journal of Machine Tools \& Manufacture, 2014, 78: 8-17.

[44] KONG L, CHEUNG C F. Prediction of surface generation in ultra-precision raster milling of optical freeform surfaces using an integrated kinematics error model[J]. Advances in Engineering Software, 2012, 45(1): $124-136$

[45] 曹义. 超精密铣削加工自由曲面光学组件误差补偿方 法[J]. 组合机床与自动化加工技术, 2018(11): 96-98.

CAO Yi. Error correction methodology for ultra-precision milling of freeform optics[J]. Modular Machine Tool \& Automatic Manufacturing Technique, 2018 (11): 96-98.

[46] ZHANG G, TO S. Cutting mechanism and surface formation of ultra-precision raster fly cutting[M]// ZHANG Junjie, GUO Bing, ZHANG Jianguo. Simulation and experiments of material-oriented ultra-precision machining. Berlin: Springer, 2019: 103-127.

[47] WANG S, TO S, CHEUNG C F. An investigation into material-induced surface roughness in ultra-precision milling $[\mathrm{J}]$. The International Journal of Advanced Manufacturing Technology, 2013, 68(1-4): 607-616.

[48] WANG S, CHEN X, TO S, et al. Modelling and prediction of the effect of cutting strategy on surface generation in ultra-precision raster milling $[\mathrm{J}]$. International Journal of Computer Integrated Manufacturing, 2017, 30(9): 895-909.

[49] SUN Z, TO S, WANG S, et al. Development of self-tuned diamond milling system for fabricating infrared micro-optics arrays with enhanced surface uniformity and machining efficiency[J]. Optics Express, 2020，28(2): 


\section{1-2237.}

[50] ZHANG S, TO S, ZHU Z, et al. A review of fly cutting applied to surface generation in ultra-precision machining $[\mathrm{J}]$. International Journal of machine tools and manufacture, 2016, 103: 13-27.

[51] ZHANG F, WANG S, AN C, et al. Full-band error control and crack-free surface fabrication techniques for ultra-precision fly cutting of large-aperture KDP crystals[J]. Frontiers of Mechanical Engineering, 2017, 12(2): 193-202.

[52] FU P, XUE J, ZHOU L, et al. Influence of the heat deformation of ultra-precision fly cutting tools on KDP crystal surface microstructure $[\mathrm{J}]$. The International Journal of Advanced Manufacturing Technology, 2019, 103(1): 1009-1018.

[53] CHEN D, ZHANG S, LIU J, et al. Morphological analysis of KDP-crystal workpiece surfaces machined by ultra-precision fly cutting[J]. Materials, 2020, 13(2): 432.

[54] WANG M, ZHANG Y, GUO S, et al. Longitudinal micro-waviness (LMW) formation mechanism on large optical surface during ultra-precision fly cutting $[J]$. The International Journal of Advanced Manufacturing Technology, 2018, 95(9-12): 4659-4669.

[55] LU H, DING Y, CHANG Y, et al. Dynamics modelling and simulating of ultra-precision fly-cutting machine tool[J]. International Journal of Precision Engineering and Manufacturing, 2020, 21(2): 189-202.

[56] CHEN D, LI S, FAN J. Effect of KDP-crystal material properties on surface morphology in ultra-precision fly cutting[J]. Micromachines, 2020, 11(9): 802.

[57] ZHU L, LI Z, FANG F, et al. Review on fast tool servo machining of optical freeform surfaces[J]. The International Journal of Advanced Manufacturing Technology, 2018, 95(5): 2071-2092.

[58] KANG M, YANG J, WANG X S, et al. Study on the variational-difference-based design and slow tool servo turning of progressive addition lenses[J]. Advances in Mechanical Engineering, 2018, 10(12): 1-12.

[59] KONG L B, CHEUNG C F. Design, fabrication and measurement of ultra-precision micro-structured freeform surfaces[J]. Computers \& Industrial Engineering, 2011, 61(1): 216-225.

[60] YU D P, WONG Y S, HONG G S. Ultraprecision machining of micro-structured functional surfaces on brittle materials[J]. Journal of Micromechanics and
Microengineering, 2011, 21(9): 10-18.

[61] LIU Q, ZHOU X Q, XU P Z, et al. A flexure-based long-stroke fast tool servo for diamond turning $[\mathrm{J}]$. International Journal of Advanced Manufacturing Technology, 2012, 59(9-12): 859-867.

[62] LI Z X, FANG F Z, ZHANG X D, et al. Highly efficient machining of non-circular freeform optics using fast tool servo assisted ultra-precision turning $[\mathrm{J}]$. Optics Express, 2017, 25(21): 25243-25256.

[63] ZHANG X D, LI Z X, ZHANG G X. High performance ultra-precision turning of large-aspect-ratio rectangular freeform optics[J]. Cirp Annals-Manufacturing Technology, 2018, 67(1): 543-546.

[64] CHEN C C, CHENG Y C, HSU W Y, et al. Slow tool servo diamond turning of optical freeform surface for astigmatic contact lens[C]//Conference on Optical Manufacturing and Testing Ix, San Diego, CA(US), 2011: 812617 .

[65] HUANG P, WU X, TO S, et al. Deterioration of form accuracy induced by servo dynamics errors and real-time compensation for slow tool servo diamond turning of complex-shaped optics[J]. International Journal of Machine Tools and Manufacture，2020， 154: 103556.

[66] 林泽钦, 陈新度, 颜志涛, 等. 微透镜阵列的慢刀伺服 加工表面粗糙度预测模型 $[\mathrm{J}]$. 哈尔滨理工大学学报, 2020, 25(3): 83-87.

LIN Zeqin, CHEN Xindu, YAN Zhitao, et al. The surface roughness prediction model of microlens array in slow tool servo cutting[J]. Journal of Harbin University of Science and Technology, 2020, 25 (3): 83-87.

[67] 李荣涁, 张志辉, 杜雪, 等. 自由曲面光学组件的设计、 加工及面形测量的集成制造技术 $[\mathrm{J}]$. 机械工程学报, 2010, 46(11): 137-148.

LEE Wingbun, CHEUNG Chifai, TO Suet, et al. Integrated manufacturing technology for design, machining and measurement of freeform optics[J]. Journal of Mechanical Engineering, 2010, 46 (11): 137-148.

[68] HUANG W H, YU D P, CHEN D S, et al. Investigation of variable spindle speed in slow tool servo-based turning of noncircular optical components[C/CD]//Eighth International Symposium on Advanced Optical Manufacturing Technologies, 2016.

[69] LIU Y T, QIAO Z, QU D, et al. Experimental investigation on form error for slow tool servo diamond turning of micro lens arrays on the roller mold[J]. 
Materials, 2018, 11(10): 1816.

[70] YAN G P, FANG F Z. Fabrication of optical freeform molds using slow tool servo with wheel normal grinding $[\mathrm{J}]$. Cirp Annals-Manufacturing Technology, 2019, 68(1): 341-344.

[71] KONG L B, MA Y G, REN M J, et al. Generation and characterization of ultra-precision compound freeform surfaces[J]. Science Progress, 2020, 103(1): 1-10.

[72] 庄司克雄. 磨削加工技术[M]. 北京: 机械工业出版社, 2007.

ZHUANGSI Kexiong. Grinding technology[M]. Beijing: China Machine Press, 2007.

[73] SAEKI M, KURIYAGAWA T, SYOJI K. Machining of aspherical molding dies utilizing parallel grinding $\operatorname{method}[\mathrm{J}]$. Journal of the Japan Society for Precision Engineering, 2002, 68(8): 1067-1071.

[74] HWANG Y, KURIYAGAWA T, LEE S K. Wheel curve generation error of aspheric microgrinding in parallel grinding method[J]. International Journal of Machine Tools and Manufacture, 2006, 46(15): 1929-1933.

[75] WANG Z, GUO J. Research on an optimized machining method for parallel grinding of $\mathrm{f}-\theta$ optics[J]. The International Journal of Advanced Manufacturing Technology, 2015, 80(5): 1411-1419.

[76] 李洪亮. 微小球面和非球面组件的纳米级磨削加工技 术研究[D]. 哈尔滨: 哈尔滨工业大学, 2011.

LI Hongliang. Research on the nanogrinding technology of micro spherical and aspherical parts[D]. Harbin: Harbin Institute of Technology, 2011.

[77] SUZUKI H, KURIYAGAWA T, SYOJI K, et al. Study on ultra-precision grinding of micro aspherical surface[J]. Journal of the Japan Society for Precision Engineering, 1998, 64(9): 1350-1354.

[78] XI J, ZHAO H, LI B, et al. Profile error compensation in cross-grinding mode for large-diameter aspheric mirrors[J]. The International Journal of Advanced Manufacturing Technology, 2016, 83(9-12): 1515-1523.

[79] 王永强, 尹韶辉, 李林升, 等. 小口径非球面光学模具 斜轴磨削加工试验研究[J]. 南华大学学报, 2016, 30(4): 49-55.

WANG Yongqiang, YIN Shaohui, LI Linsheng, et al. Experimental study on inclined rotational grinding for small aspherical optical mould[J]. Journal of University of South China, 2016, 30(4): 49-55.

[80] 陈逢军, 尹韶辉, 胡天, 等. 微小非球面光学模具单点
斜轴误差补偿磨削[J]. 机械工程学报, 2013, 49(17): 59-64.

CHEN Fengjun, YIN Shaohui, HU Tian, et al. Single-point inclined-axis error compensation grinding for small aspheric optical molds[J]. Journal of Mechanical Engineering, 2013, 49(17): 59-64.

[81] SUZUKI H, KODERA S, MAEKAWA S, et al. Study on precision grinding of micro aspherical surface-Feasibility study of micro aspherical surface by inclined rotational grinding[J]. Journal of the Japan Society for Precision Engineering, 1998, 64(4): 619-623.

[82] 王泽彪. 微小非球面碳化铇模具的斜轴磨削及超声抛 光工艺研究[D]. 长沙: 湖南大学, 2011 .

WANG Zebiao. Inclined axis grinding for WC micro aspheic mould and ultrasonic vibration assisted finishing properties study[D]. Changsha: Hunan University, 2011.

[83] 刘林枝. 小口径非球面斜轴磨削的砂轮磨损及补偿研 究[J]. 中国机械工程，2012，23(15): 1789-1792.

LIU Linzhi. Wear compensation of grinding wheel for inclined-axis grinding small aspherical surface[J]. China Mechanical Engineering, 2012， 23 (15): 1789-1792.

[84] 陈逢军, 尹韶辉, 胡天. $\Phi 2 \mathrm{~mm}$ 非球面光学模具斜轴磨削 补偿研究[OL]. [2013-10-05]. http://www.paper. edu.cn. CHEN Fengjun, YIN Shaohui, HU Tian. Inclined-axis error compensation grinding for $\Phi 2 \mathrm{~mm}$ aspheric optical mould[OL]. [2013-10-05]. http://www.paper. edu.cn.

[85] 尹韶辉, 龚胜, 何博文, 等. 小口径非球面斜轴磨削及 磁流变抛光组合加工工艺及装备技术研究 $[\mathrm{J}]$. 机械工 程学报, 2018, 54(21): 205-211.

YIN Shaohui, GONG Sheng, HE Bowen, et al. Development on synergistic process and machine tools integrated inclined axis grinding and magnetorheological polishing for small aspheric surface[J]. Journal of Mechanical Engineering, 2018, 54 (21): 205-211.

[86] LIU K, LI X, RAHMAN M. Characteristics of ultrasonic vibration-assisted ductile mode cutting of tungsten carbide[J]. The International Journal of Advanced Manufacturing Technology, 2008, 35(7-8): 833-841.

[87] MAHADDALKAR P M, MILLER M H. Force and thermal effects in vibration-assisted grinding $[\mathrm{J}]$. The International Journal of Advanced Manufacturing Technology, 2014, 71(5-8): 1117-1122.

[88] FANG F, NI H, GONG H. Rotary ultrasonic machining of hard and brittle materials[J]. Nanotechnol. Precis. Eng., 2014, 12(3): 227-234. 
[89] JIAN H Z, YAN Z, FU Q T, et al. Kinematics and experimental study on ultrasonic vibration-assisted micro end grinding of silica glass[J]. The International Journal of Advanced Manufacturing Technology, 2015, 78(9-12): 1893-1904.

[90] FENG P, LIANG G , ZHANG J. Ultrasonic vibration-assisted scratch characteristics of silicon carbide-reinforced aluminum matrix composites[J]. Ceramics International, 2014，40(7): 10817-10823.

[91] 赵培轶. BK7 光学玻璃超声振动磨削脆塑性转变及加 工质量研究[D]. 哈尔滨: 哈尔滨工业大学, 2017.

ZHAO Peiyi. Research on brittle ductile transition and machining quality in ultrasonic vibration assisted grinding of BK7 optical glass[D]. Harbin: Harbin Institute of Technology, 2017.

[92] ZHOU M, ZHAO P. Prediction of critical cutting depth for ductile-brittle transition in ultrasonic vibration assisted grinding of optical glasses[J]. The International Journal of Advanced Manufacturing Technology, 2016, 86(5): 1775-1784.

[93] 孙国燕. 低膨胀光学玻璃的超声振动磨削机理及工艺 技术研究[D]. 西安: 中国科学院西安光学精密机械研 究所, 2019 .

SUN Guoyan. Ultrasonic vibration assisted grinding mechanism and rocessing technology on low expansion optical glass[D]. Xi'an: Xi'an Institute of Optics and Percision Mechanics of CAS, 2019.

[94] 郭兵. 超硬微结构光学菜单面的超声振动磨削加工技 术研究[D]. 哈尔滨: 哈尔滨工业大学, 2012.

GUO Bing. Research on ultrasonic vibration assistant grinding of super-hard microstructured optical surfaces[D]. Harbin: Harbin Institute of Technology, 2012.

[95] JIANG C, WU T, YE H, et al. Estimation of energy and time savings in optical glass manufacturing when using ultrasonic vibration-assisted grinding $[\mathrm{J}]$. International Journal of Precision Engineering and Manufacturing-Green Technology, 2019，6(1): 1-9.

[96] 许陆昕. 碳化硅陶瓷超声振动磨削表面质量研究[D]. 苏州: 苏州科技大学, 2019 .

$\mathrm{XU}$ Luxin. Study on surface quality of ultrasonic vibration grinding of $\mathrm{SiC}$ ceramics[D]. Suzhou: Suzhou University of Science and Technology, 2019.

[97] 周莲. $\mathrm{SiC}$ 光学组件超声振动磨削系统设计与研究 $[\mathrm{D}]$. 苏州: 苏州科技大学, 2018 .
ZHOU Lian. Design and research of ultrasonic vibration grinding system for $\mathrm{SiC}$ optical elements[D]. Suzhou: Suzhou University of Science and Technology, 2018.

[98] 姜振华. 光学大镜加工中弧面磨削几何学原理及其实 现方法[D]. 上海: 上海交通大学, 2017.

JIANG Zhenghua. Geometrical principle and realization of toric grinding for large optical mirrors[D]. Shanghai: Shanghai Jiao Tong University, 2017.

[99] 林硕. 大口径非球面镜少轴弧面包络磨削关键技术研 究[D]. 上海: 上海交通大学, 2019.

LIN Shuo. Study on the key technologies of fewer-axis arc envelope grinding for large aspherical surfaces[D]. Shanghai: Shanghai Jiao Tong University, 2019.

[100] NAMBA Y, SHIMOMURA T, FUSHIKI A, et al. Ultra-precision polishing of electroless nickel molding dies for shorter wavelength applications[J]. CIRP Annals, 2008, 57(1): 337-340.

[101] CHEUNG C, KONG L, HO L, et al. Modelling and simulation of structure surface generation using computer controlled ultra-precision polishing $[\mathrm{J}]$. Precision Engineering, 2011， 35(4): 574-590.

[102] 廖文林, 戴一帆, 周林, 等. 离子束作用下的光学表 面粗糙度演变研究 $[\mathrm{J}]$. 应用光学, 2010, 31(6): 1041-1045.

LIAO Wenlin, DAI Yifan, ZHOU Lin, et al. Optical surface roughness in ion beam process[J]. Journal of Applied Optics, 2010, 31 (6): 1041-1045.

[103] ZEUNER M , KIONTKE S. Ion beam figuring technology in optics manufacturing: An established alternative for commercial applications[J]. Optik \& Photonik, 2012, 7(2): 56-58.

[104] 孟晓辉, 王永刚, 李文卿. 航天相机非球面光学组件 的离子束抛光工艺研究 $[J]$. 航天制造技术, 2016(6): 27-31.

MENG Xiaohui, WANG Yonggang, LI Wenqing. Application of ion beam figuring technology on aerospace camera aspheric[J]. Aerospace Manufacturing Technology, 2016(6): 27-31.

[105] 徐明进, 戴一帆, 周林, 等. 离子束抛光热场数值仿 真分析[J]. 航空精密制造技术，2013，49(4)：18-21.

XU Mingjin, DAI Yifan, ZHOU Lin. Numerical simulation of thermal field in ion-beam figuring $[\mathrm{J}]$. Aviation Precision Manufacturing Technology, 2013, 49 (4): $18-21$.

[106] 张云飞, 吉方, 蓝河, 等. 离子束抛光热效应研究 $[J]$. 
现代制造工程，2013(9)：83-86.

ZHANG Yunfei, JI Fang, LAN He, et al. Study on the thermal effect in the ion beam figuring $[\mathrm{J}]$. Modern Manufacturing Engineering, 2013(9): 83-86.

[107] 唐瓦, 邓伟杰, 李锐钢, 等. 离子束抛光高陡度离轴 非球面的去除函数修正 $[\mathrm{J}]$. 光学精密工程, 2015 , 23(6): 1572-1579.

TANG Wa, DENG Weijie, LI Ruigang, et al. Correction of removal function of ion beam figuring highly steep off-axi asphere[J]. Optics and Precision Engineering, 2015, 23 (6): 1572-1579.

[108] 张厚亮, 吕学超, 任大鹏, 等. $A r+$ 离子束对 $U$ 薄膜表 面粗䊁度的影响 [J]. 稀有金属材料与工程, 2010 , 39(5): 889-891.

ZHANG Houliang, LÜ Xuechao, REN Dapeng, et al. Influence of $\mathrm{Ar}+$ ion beam sputter on surface roughness of uranium film[J]. Rare Metal Materials and Engineering, 2010, 39 (5): 889-891.

[109] 冯殊瑞, 解旭辉, 周林, 等. 离子束加工 KDP 晶体材 料表面粗粘度演变 [J]. 航空精密制造技术, 2012, 48(6): 10-12, 17.

FENG Shurui, XIE Xuhui, ZHOU Lin, et al. Evolvement on surface roughness of KDP crystal in ion beam figuring[J]. Aviation Precision Manufacturing Technology, 2012, 48 (6): 10-12, 17.

[110] 武否, 刘卫国, 蔡长龙, 等. $\mathrm{ZnS}$ 离子束抛光过程中的 粗䊁度演变 $[\mathrm{J}]$. 西安工业大学学报, 2014, 34(12): 947-952.

WU Lei, LIU Weiguo, CAI Changlong, et al. Roughness evolution research of $\mathrm{ZnS}$ by ion beam polishing process[J]. Journal of Xi' an Technological University, 2014, 34(12): 947-952.

[111] DEMMLER M, ZEUNER M, ALLENSTEIN F, et al. Ion beam figuring (IBF) for high precision optics[C]//Advanced Fabrication Technologies for Micro/Nano Optics and Photonics III, 2010: 75910Y.

[112] 尹小林. 空间复杂光学曲面离子束修形设备与工艺研 究[D]. 长春: 中国科学院长春光学精密机械与物理研 究所, 2017.

YIN Xiaolin. Research on equipment and technology of ion beam figuring for space complex optical surface[D]. Changchun: Changchun Institute of Optics, Fine Mechanics and Physics of CAS, 2017.

[113] 伍艺龙. 等离子体抛光机理的研究 $[D]$. 西安: 西安工 业大学, 2011.
WU Yilong. Research on the mechanism of plasma polishing[D]. Xi'an: Xi'an Technological University, 2011.

[114] ZHANG J, LI B, WANG B, et al. Analysis on formation mechanism of ultra-smooth surfaces in atmospheric pressure plasma polishing[J]. The International Journal of Advanced Manufacturing Technology , 2013, 65(9-12): 1239-1245.

[115] ARNOLD T, BÖHM G. Application of atmospheric plasma jet machining (PJM) for effective surface figuring of $\mathrm{SiC}[\mathrm{J}]$. Precision Engineering, 2012, 36(4): 546-553.

[116] LALIOTIS A, TRUPKE M, COTTER J, et al. ICP polishing of silicon for high-quality optical resonators on a chip[J]. Journal of Micromechanics and Microengineering, 2012, 22(12): 125011.

[117] CASTELLI M, JOURDAIN R, MORANTZ P, et al. Rapid optical surface figuring using reactive atom plasma[J]. Precision Engineering, 2012, 36(3): 467-476.

[118] DENG H, YAMAMURA K. Smoothing of reaction sintered silicon carbide using plasma assisted polishing[J]. Current Applied Physics, 2012, 12: 24-28.

[119] WU Y, LIU W, HANG L. Research on RF-generated plasma polishing[J]. Physics Procedia, 2012, 32 : 590-597.

[120] 王东方. 大气等离子体加工熔石英技术研究 [J]. 陕西 理工学院学报, 2013(2): 1-5.

WANG Dongfang. Research on atmospheric pressure plasma processing technology of fused silicon[J]. Journal of Shaanxi University of Technology, 2013(2): $1-5$.

[121］史宝鲁. $\mathrm{SiC}$ 反射镜电弧增强等离子体加工关键技术 研究[D]. 长沙：国防科学技术大学，2016.

SHI Baolu. Study on key techniques in arc-enhanced plasma machining for silicon carbide mirrors[D]. Changsha: National University of Defense Technology, 2016.

[122] PAETZELT H, BÖHM G, ARNOLD T. Etching of silicon surfaces using atmospheric plasma jets[J]. Plasma Sources Science and Technology, 2015, 24(2): 025002.

[123] 夏龙光. 光学组件大气等离子体高精度确定性加工技 术研究[D]. 哈尔滨：哈尔滨工业大学, 2018.

XIA Longguang. Research on atmospheric plasma high precision deterministic processing technologies of optical elements[D]. Harbin : Harbin Institute of 
Technology, 2018.

[124] SU X, JI P, LIU K, et al. Combined processing chain for freeform optics based on atmospheric pressure plasma processing and bonnet polishing[J]. Optics Express, 2019, 27(13): 17979-17992.

[125] YAMAMURA K, TAKEDA Y, SAKAIYA S, et al. High-spatial resolution figuring by pulse width modulation controlled plasma chemical vaporization machining[J]. Procedia CIRP, 2016, 42(1): 508-511.

[126] JOURDAIN R, CASTELLI M, SHORE P, et al. Reactive atom plasma (RAP) figuring machine for meter class optical surfaces[J]. Production Engineering, 2013, 7(6): 665-673.

[127] BINGHAM R G, WALKER D D, KIM D H, et al. Novel automated process for aspheric surfaces[C]//Current Developments in Lens Design and Optical Systems Engineering, 2000: 445-450.

[128] 王策. 适应自由曲面曲率的气囊抛光残留高度量化理 论研究[D]. 秦皇岛: 燕山大学, 2019.

WANG Ce. Theoretical study on quantification of bonnet polishing residue height adapted to free surface curvature[D]. Qinhuangdao: Yanshan University, 2019.

[129] BO Z, XIANHUA C, RI P, et al. The effect of tool wear on the removal characteristics in high-efficiency bonnet polishing[J]. The International Journal of Advanced Manufacturing Technology, 2017, 91(9-12): 3653-3662.

[130] PAN R, ZHONG B, WANG Z, et al. Influencing mechanism of the key parameters during bonnet polishing process $[\mathrm{J}]$. The International Journal of Advanced Manufacturing Technology, 2018, 94(1-4): 643-653.

[131] CAO Z C, CHEUNG C F. Multi-scale modeling and simulation of material removal characteristics in computer-controlled bonnet polishing[J]. International Journal of Mechanical Sciences, 2016, 106: 147-156.

[132] SHI C, PENG Y, HOU L, et al. Micro-analysis model for material removal mechanisms of bonnet polishing[J]. Applied Optics, 2018， 57(11): 2861-2872.

[133] SHI C, PENG Y, HOU L, et al. Improved analysis model for material removal mechanisms of bonnet polishing incorporating the pad wear effect[J]. Applied Optics, 2018, 57(25): 7172-7186.

[134] ZHONG B, XU Q, WANG J, et al. Evaluation and compensation of a kinematic error to enhance prepolishing accuracy for large aspheric surfaces by robotic bonnet technology[J]. Optics Express, 2020, 28(17): 25085-25100.

[135] QIN K, LIU Z, LIU J. Research on collision detection algorithm of multi-robot bonnet polishing system[C]//AOPC 2020 : Optics Ultra Precision Manufacturing and Testing, 2020: 1156815.

[136] YU T B, WANG Z H, GUO X P, et al. Effect of ultrasonic vibration on polishing monocrystalline silicon: Surface quality and material removal rate[J]. International Journal of Advanced Manufacturing Technology, 2019, 103(5-8): 2109-2119.

[137] YU T B, AN J H, YANG X Z, et al. The study of ultrasonic vibration assisted polishing optical glass lens with ultrasonic atomizing liquid[J]. Journal of Manufacturing Processes, 2018, 34: 389-400.

[138] EL-HOFY H. Vibration-assisted electrochemical machining : A review[J]. International Journal of Advanced Manufacturing Technology, 2019, 105(1-4): 579-593.

[139] LI Y G, WU Y B, ZHOU L B, et al. Vibration-assisted dry polishing of fused silica using a fixed-abrasive polisher[J]. International Journal of Machine Tools \& Manufacture, 2014, 77: 93-102.

[140] 李晓奥, 彭小强, 胡皓. 振动辅助抛光的原理分析与 实验研究 $[J]$, 航空精密制造技术, 2016, 52(4): 14-19. LI Xiaoao, PENG Xiaoqiang, HU Hao. Principle analysis and experimental study of vibration assisted polishing $[\mathrm{J}]$. Aviation Precision Manufacturing Technology, 2016, 52 (4): 14-19.

[141] 李晓奥. 光学零件振动辅助抛光关键技术研究 $[D]$. 长 沙: 国防科学技术大学, 2016 .

LI Xiaoao. Study on the key technology of vibration-assisted polishing for optical elements[D]. Changsha: National University of Defense Technoloy, 2016.

[142] 武昌壕. 超硬微结构光学表面振动辅助抛光工艺研究 [D]. 哈尔滨: 哈尔滨工业大学, 2013.

WU Changhao. Ultrasonic assisted chemical mechanical polishing of superhard micro-structured optical surface[D]. Harbin: Harbin Institute of Technology, 2013.

[143] YU T B, ZHANG T Q, YU X M, et al. Study on optimization of ultrasonic-vibration-assisted polishing process parameters[J]. Measurement, 2019, 135 : 651-660. 
[144] SUZUKI H, HAMADA S, OKINO T, et al. Ultraprecision finishing of micro-aspheric surface by ultrasonic two-axis vibration assisted polishing[J]. Cirp Annals-Manufacturing Technology, 2010 , 59(1) : 347-350.

[145] 冯洁. 振动辅助磁流变抛光关键技术研究 [D]. 长春: 长春工业大学， 2020 .

FENG Jie. Research on key technologies of vibration-assisted magnetorheological polishing[D]. Changchun: Changchun University of Technology, 2020.

[146] SONG D L, ZHAO J, JI S J, et al. Development of a novel two-dimensional ultrasonically actuated polishing process[J]. Aip Advances, 2016, 6(11): 115105.

[147] GU Yan, CHEN Xiuyuan, LIN Jieqiong, et al. Vibration-assisted roll-type polishing system based on compliant micro-motion stage micromachines[J]. Micromachines, 2018, 9(10): 1204-1208.

[148] GU Y, DUAN X X, LIN J Q, et al. Design, analysis, and testing of a novel 2-DOF vibration-assisted polishing device driven by the piezoelectric actuators[J]. International Journal of Advanced Manufacturing Technology, 2020, 111(1-2): 471-493.

[149] CHEE S K, SUZUKI H, OKADA M, et al. Precision polishing of micro mold by using piezoelectric actuator incorporated with mechanical amplitude magnified mechanism[C]//Advanced Materials Research, 2011: 470-475.

[150] CHEE S K, SUZUKI H, UEHARA J, et al. Preliminary studies for precision polishing of micro structured mold by using three-dimensional low frequency vibration utilizing piezoelectric actuator incorporated with mechanical amplitude magnified mechanism[C]// Advanced Materials Research, 2012: 231-236.

[151] WANG G, LV B, ZHENG Q, et al. Polishing trajectory planning of three-dimensional vibration assisted finishing the structured surface[J]. AIP Advances, 2019, 9(1): 015012.

[152] GUO J, MORITA S Y, HARA M, et al. Ultra-precision finishing of micro-aspheric mold using a magnetostrictive vibrating polisher[J]. CIRP Annals, 2012, 61(1): 371-374.

[153] GUO J, SUZUKI H, MORITA S, et al. Micro polishing of tungsten carbide using magnetostrictive vibrating polisher[J]. Key Engineering Materials, 2012, 516: 569-574.
[154] GUO J, KUM C W, AU K H, et al. New vibration-assisted magnetic abrasive polishing (VAMAP) method for microstructured surface finishing[J]. Optics Express, 2016, 24(12): 13542-13554.

[155] GUO J, JONG H J H, KANG R, et al. Novel localized vibration-assisted magnetic abrasive polishing method using loose abrasives for V-groove and Fresnel optics finishing[J]. Opt Express， 2018， 26(9): 11608-11619.

[156] MADRAKI Y, HORMOZI S, OVARLEZ G, et al. Enhancing shear thickening[J]. Physical Review Fluids, 2017, 2(3): 033301.

[157] BROWN E, JAEGER H M. Shear thickening in concentrated suspensions: Phenomenology, mechanisms and relations to jamming $[\mathrm{J}]$. Reports on Progress in Physics, 2014, 77(4): 046602.

[158] LI M, HUANG Z, DONG T, et al. Surface integrity of bearing steel element with a new high-efficiency shear thickening polishing technique[J]. Procedia CIRP, 2018, 71: 313-316.

[159] LI M, HUANG Z, DONG T, et al. Surface quality of Zirconia $\left(\mathrm{ZrO}_{2}\right)$ parts in shear-thickening high-efficiency polishing[J]. Procedia CIRP, 2018, 77: 143-146.

[160] LYU B, HE Q, CHEN S, et al. Experimental study on shear thickening polishing of cemented carbide insert with complex shape[J]. The International Journal of Advanced Manufacturing Technology, 2019, 103(1-4): 585-595.

[161] ZHU W L, BEAUCAMP A. Non-newtonian fluid based contactless sub-aperture polishing[J]. CIRP Annals, 2020, 69(1): 293-296.

[162] WAGNER N J, BRADY J F. Shear thickening in colloidal dispersions[J]. Physics Today, 2009, 62(10): 27-32.

[163] 秦琳, 弥谦, 李宏. 基于非牛顿流体新型液浮法抛光 技术研究 $[J]$. 应用光学，2019，40(2)：223-228.

QIN Lin, MI Qian, LI Hong. Novel liquid float polishing technology based on non-newtonian fluid[J]. Journal of Applied Optics, 2019， 40(2): 223-228.

[164] WU Y, CAO S, XUAN S, et al. High performance zeolitic imidazolate framework-8 (ZIF-8) based suspension: Improving the shear thickening effect by controlling the morphological particle-particle interaction[J]. Advanced Powder Technology, 2020, 31(1): 70-77.

[165] LI Min, LIU Minghui, RIEMER O, et al. Anhydrous 
based shear-thickening polishing of KDP crystal[J]. Chinese Journal of Aeronautics , 2020 , https://doi.org/10.1016/j.cja.2020.09.019.

[166] 陈士豪, 吕冰海, 贺乾坤, 等. 圆柱曲面剪切增稠抛 光材料去除函数仿真与实验研究 [J]. 表面技术, 2019 , 48(10): 355-362.

CHEN Shihao, LÜ Binghai, HE Qiankun, et al. Simulation and experimental study on material removal function of shear thickening polishing cylindrical surface[J]. Surface Technology, 2019, 48(10): 355-362.

[167] 翁海舟, 吕冰海, 胡刚翔, 等. 石英芯片剪切增稠抛 光优化实验 $[\mathrm{J}]$. 纳米技术与精密工程, 2017, 15(3): 227-233.

WENG Haizhou, LÜ Binghai, HU Gangxiang, et al. Optimization experiments for shear thickening polishing of quartz substrates[J]. Nanotechnology and Precision Engineering, 2017, 15(3): 227-233.

[168] 李霜兵. $\mathrm{SiO}_{2}$ 剪切增稠流体的制备及其应用研究 $[D]$. 天津: 天津大学, 2017.

LI Shuangbing. Preparation and application of high-performance $\mathrm{SiO}_{2}$ shear thickening fluids[D]. Tianjin: Tianjin University, 2017.

[169] LI M, KARPUSCHEWSKI B, OHMORI H, et al. Adaptive shearing-gradient thickening polishing (AS-GTP) and subsurface damage inhibition[J]. International Journal of Machine Tools and Manufacture, 2021, 160(1): 103651.

[170] 翁海舟. 剪切增稠与电解复合高效抛光方法基础研究 [D]. 杭州: 浙江工业大学, 2017.

WENG Haizhou. Basic study on high efficiency electrolysis compounded shear thickening polishing method[D]. Hangzhou : Zhejiang University of Technology, 2017.

[171] WEI H, GAO H, WANG X. Development of novel guar gum hydrogel based media for abrasive flow machining: Shear-thickening behavior and finishing performance[J]. International Journal of Mechanical Sciences, 2019, 157: $758-772$.

[172] MORI Y, YAMAUCHI K, ENDO K. Elastic emission machining[J]. Precision Engineering, 1987, 9(3) : 123-128

[173] 彭文强. 基于材料弹性域去除的超光滑表面加工关键 技术研究[D]. 长沙：国防科学技术大学，2014.

PENG Wenqiang. Study on the key technology of ultrasmooth surface fabrication based on the material removal in elastic mode[D]. Changsha : National University of Defense Technology, 2014.

[174] HASHIMOTO F, YAMAGUCHI H, KRAJNIK P, et al. Abrasive fine-finishing technology[J]. CIRP Annals, 2016, 65(2): 597-620.

[175] 李岩. 基于中心供液流体动压原理的盘式抛光理论与 实验研究[D]. 天津: 天津大学, 2017.

LI Yan. Theoretical and experimental study on disc polishing technology based on center-inlet hydrodynamic theory[D]. Tianjin: Tianjin University, 2017.

[176] 胡绍波. 面向光学玻璃的精密加工技术研究[D]. 上 海: 东华大学, 2011 .

HU Shaobo. Study of flat flexible platform based on pneumatic proportional control system[D]. Shanghai: Donghua University, 2011.

[177] 刘笑. RB-SiC 反射镜 $\mathrm{Si}$ 改性层的粘弹性流体超光滑抛 光技术研究[D]. 济南: 山东大学, 2019 .

LIU Xiao. Utra-smooth polishing of si modification layer on sic surface with viscoelastic fluid[D]. Jinan: Shandong University, 2019.

[178] 刘文龙. 基于 $\operatorname{CCOS}$ 技术的盘式动压抛光工艺理论研 究[D]. 天津: 天津大学, 2014.

LIU Wenlong. Theoretical research on disc hydrodynamic polishing based on CCOS[D]. Tianjin : Tianjin University, 2014.

[179] J彦飞. 盘式动压抛光理论及实验研究[D]. 天津: 天 津大学, 2014 .

DIAO Yanfei. Theoretical and experimental research on disc hydrodynamic polishing[D]. Tianjin : Tianjin University, 2014.

[180] 薛凯元. 线性液动压抛光加工流体动压特性研究[D]. 杭州: 浙江工业大学, 2019.

XUE Kaiyuan. Study on hydrodynamic characteristics of linear hydrodynamic polishing[D]. Hangzhou: Zhejiang University of Technology, 2019.

[181] 朱胜伟. 可控边界流体抛光的数值模拟和试验研究 [D]. 杭州: 浙江工业大学, 2016.

ZHU Shengwei. Simulation and experimental study of controllable border hydropolishing[D]. Hangzhou : Zhejiang University of Technology, 2016.

[182] WEN D H, PIAO Z Y, ZHANG T H. A hydrodynamic suspension polishing method for ultrasmooth and low-damage surface[J]. Precision Engineering, 2016, 46: $278-287$. 
[183] TAKEI Y, MIMURA H. Effect of focusing flow on stationary spot machining properties in elastic emission machining[J]. Nanoscale Research Letters, 2013, 8(1): 1-6.

[184] 李庆宇. 基于流体动力润滑效应的双转弹性发射加工 技术研究[D]. 长沙：国防科学技术大学，2015.

LI Qingyu. Study on the technology of elastic emission machining with dual-rotor based on hydrodynamic lubrication[D]. Changsha : National University of Defense Technology, 2015.

[185] 郑子军, 薛凯元, 文东辉, 等. 线性液动压抛光加工 的流体动压特性研究 [J]. 中国机械工程, 2020, 31(8): 907-914

ZHENG Zijun, XUE Kaiyuan, WEN Donghui, et al. Study on hydrodynamic pressure characteristics of linear hydrodynamic pressure polishing $[\mathrm{J}]$. China Mechanical Engineering, 2020, 31(8): 907-914.

[186] HIRATA T, TAKEI Y, MIMURA H. Machining property in smoothing of steeply curved surfaces by elastic emission machining[J]. Procedia CIRP, 2014, 13: 198-202.

[187] KANAOKA M, NOMURA K, YAMAUCHI K, et al. Efficiency-enhanced elastic emission machining on the basis of processing mechanism $[\mathrm{C}] / /$ Proceedings of the 12th Euspen International Conference, Stockholm, 2012.
[188] PENG W, LI Y, WANG Z, et al. Experimental exploration of the hydrodynamic effect polishing machinability for different types of material[C]//IOP Conference Series: Materials Science and Engineering, 2018: 012100

[189] WANG C, YANG W, WANG Z, et al. Highly efficient deterministic polishing using a semirigid bonnet[J]. Optical Engineering, 2014, 53(9): 095102.

[190）李晨晨. 基于 CCOS 的双面抛光工艺算法研究[D]. 长 春：长春理工大学, 2020.

LI Chenchen. Research on double-sided polishing process algorithm based on CCOS[D]. Changchun: Changchun University of Science and Technology, 2020.

[191] 邢进. 光学非球面 CCOS 抛光理论与技术研究[D]. 长 春：吉林大学， 2020 .

XING Jin. Research on the CCOS polishing theory and technology of optical aspheric surface[D]. Changchun: Jilin University, 2020.

作者简介: 王振忠, 男, 1981 年出生, 博士, 副教授。主要研究方向为 超精密磨削、抛光装备开发及工艺技术。

E-mail: wangzhenzhong@xmu.edu.cn

郭江(通信作者), 男, 1982 年出生, 博士, 教授, 博士研究生导师。主 要研究方向为精密与超精密加工、中子光学、执行器、机电一体化。

E-mail: guojiang@dlut.edu.cn 